\title{
Chimpanzees behavioral response to a shift in number of visitors during autumn holiday in Aalborg zoo
}

\author{
Ottosen MP1,2, Kjær JW', , Andersen M, 1,2, Bach LA ${ }^{1,2}$, Pagh $S^{1,2}$ \\ ${ }^{I}$ Department of Chemistry and Bioscience - Section of Biology and Environmental Science, Aalborg \\ University, Fredrik Bajers Vej 7, 9220 Aalborg, Denmark \\ ${ }^{2}$ Aalborg Zoo, Mфlleparkvej 63, 9000 Aalborg, Denmark
}

Corresponding Author: Sussie Pagh, Department of Chemistry and Bioscience - Section of Biology and Environmental Science, Aalborg University, Fredrik Bajers Vej 7, 9220 Aalborg, Denmark; Email: sup@bio.aau.dk

\begin{abstract}
This study examine how the number of visitors affects the behavior of four chimpanzees Pan troglodytes in Aalborg Zoo. Overall, continuous focal sampling showed no significant changes in the chimpanzee's behaviors that could be linked to the number of visitors. However, the youngest individual showed significant more shifts in behavior during holidays than during the control days. Individual differences were found in behavioural interactions, fouraging and stereotypical behavior.
\end{abstract}

Keywords: animal personality, captivity, behaviour, welfare, Pan troglodytes

\section{Introduction}

Natural Environment For Chimpanzees

Chimpanzees (Pan troglodytes verus) live in numerous different habitats throughout the equatorial part of Africa (Stanford, 1998). The natural habitat of chimpanzee vary from deep forests to open mixed forestsavannas in both lowlands and in relatively high altitudes (AZA Ape TAG, 2010). The size of the chimpanzee's communities in the wild are determined by their habitat, such as the density of the forest and the accessibility to food. The distribution of females and males in wild chimpanzee communities are typically relatively even (AZA Ape TAG, 2010). Boesch (1996) studied the group size of several wild chimpanzee communities, and found a mean group size of 5.7 individuals. However, the size of chimpanzee communities may be as large as 20-100 individuals (AZA Ape TAG, 2010). Chimpanzees are living in societies with competition among goup members as well as between groups, but also with high level of cooperation between individuals (de Waal, 2005). 


\section{Chimpanzees In Captivity}

Chimpanzees in zoos play an important role in species conservation by acting as ambassadors for their wildliving counterparts. Captive chimpanzees are used for education and research, and as genetically population assurance if the wild population gets critically low (Ralls and Ballou, 1992; Lecturer and Booth, 2013; Penfold et al., 2013; Skibins and Powell, 2013). The western chimpanzee is losing its habitats, including rainforest and savanna woodland habitat. Chimpanzees are still hunted illegally primarily for food and because they may damage crops, and especially infant chimpanzees are caught and traded by wildlife dealers (Kühl et al., 2017).

Knowledge and understanding of the natural behaviour of animals in zoos is important in order to secure the wellbeing of animals (Kreger and Mench, 1995; Hosey, 2005). One way to achieve welfare for captive animals is to secure acess to natural behaviour as e.g. foraging and interact with conspecifics and to avoid stress from a changing environment. Across varying animal species research has shown that deprivation of behaviour, typical for the species, is correlated with reduced welfare e.g hens deprived of sand to dust bath can lead to self- or allopecking (Vestergaard and Lawson, 1997; Clubb and Mason, 2006; Pomerantz and Terkel, 2013; Cronin, West and Ross., 2016). Studies show that wild chimpanzees spend about half of their life sleeping and resting, therefore captive chimpanzees must be provided with elevated sleeping platforms or beds and bedding material that contributes to appropriate sleep (Pruetz and McGrew, 2001). Chimpanzees in the wild live in fission-fusion social systems in contrast to the relatively permanent social groups in most zoos (Pruetz and McGrew, 2001). According to AZA Ape TAG, (2010) chimpanzee groups should consist of multimale/multi-female groups and newly established groups are recommended to contain three males and five females (AZA Ape TAG, 2010). Another way of increasing the well being of captive individuals is enrichment of their experienced environment as for example placing objects that invite to tool-use, object-play, or foraging (Wood, 1998). As vision is essential in social and non-social life in most primates, recent research efforts have furthermore explored the effects of visual enrichment in orangutan (Pongo pygmaeus) (Garcia et al. 2020). For the well being of chimpanzees the access to outdoor facilities seems crucial judging from their behaviour and utilisation of physical features (AZA Ape TAG, 2010; Martínez, 2014). Clarke and Maple (1982) found a large reduction in stereotyped and self-directed behavior, after translocation to a naturalistic island from laboratory environments. Another study showed a decrease in attention behaviors (towards visitors) and abnormal behavior for chimpanzees when translocated from an indoor hardscape-type exhibition to a naturalistic enclosure with access to outside areas, while gorillas showed reduced agonism and attention behavior (Ross et al., 2011). The complexity and features of the enclosure seems to be more important than the space itself when creating functionally appropriate captive environments (Webb et al., 2018). It is important that the design contribute to that the chimpanzees have control over their environment by providing the opportunity for the chimpanzees to choose microenvironments (AZA Ape TAG, 2010). Hiding places are both intended to escape conspecifics and to block the view by visitors.

Several studies indicate that visitors may be a source of stress for animals, depending on the species and the behaviour of visitors (Fernandez et al., 2009). Carder and Semple (2008) found a positive association between self-scratching and number of visitors for gorillas and Wood (1998) found that many visitors caused a decrease in frequencies of foraging, grooming, play and object-using. Multiple studies indicate that zoo visitor presence, density, activity, size and position are linked to specific animal behaviour. Previous studies interpret that visitor's effect on animals can be both negative and enriching (Davey, 2007). Thompson (1976) found that sexual behaviour between chimpanzees increased when zoos visitor numbers were higher. Hosey and Druck (1987) studied 12 captive primate species, they observed that visitor presence had an effect on mobility and spatial dispersion, it was also noticed that primate behaviours was mostly directed at active visitor groups instead of the passive (Davey, 2007).

Captive chimpanzees have behaviour that is considered as abnormal. The abnormal behaviour varies but indicate possible signs of poor mental health (Birkett and Newton-Fisher, 2011, Pomerantz et al., 2013). Captive environments often lead to abnormal behaviour in several species (Birkett and Newton-Fisher, 2011). Studies shows consideratble individual variation in stress physiology (Carere et al., 2010). Inheritage and accumulated experiences through lifetime espressed as for example aggression, boldness, fearfulness, cooperativeness, and exploration tendencies may influence the behaviour of individual chimpanzees to 
different kind of disturbance from their invironement (Wolf and Wessing, 2012; Dingemanse and Reale, 2005).

The aim of this study is to evaluate a number of different behavioural expressions of four chimpanzees, housed at Aalborg zoo, in response to the number of visitors.

\section{Methods}

\section{Subjects}

This study concern the behaviour of four chimpanzees, housed in Aalborg zoo. The chimpanzee, Jutta is 45 years old and the mother of the other three chimpanzees; My a female, age of 7, Sebastian a 12 years old male, Laura a 25 years old female. Jutta was born in Africa but was moved to Aalborg Zoo when she was about 6 years old, the other chimpanzees are all born in Aalborg zoo.

\section{Enclosure}

The chimpanzee enclosure consists of an outside area around $125 \mathrm{~m} 2$, and an inside area around $100 \mathrm{~m} 2$. Both areas contain several large rocks and a large climbing tree with vertical and horizontal trunks and ropes between them allowing the chimpanzees to climb (Appendix A). Both areas have containers with small holes, containing oatmeal, for which the chimpanzees must use tools to get. In the outside enclosure there is a tire swing, a shelter, and multiple trunks on the ground. The inside area also contains wooden platforms and bedding material. Despite the are between the indoor and outdoor enclosure, the enclousures does not contain covers where chimpanzees can hide from the observing visitors.

\section{Procedure}

The behavioural observations were collected between 9.00 and 16.00 every day in two periods: 1) The 30/9 to the 6/10 and 2) The 14/10 to the 20/10. Chimpanzees in Aalborg Zoo are fed between 9.00-10.00 and is open to visitors from 10-16 on weekdays and from 10-17 on holidays and weekend. The first period was a normal week and served as a control week. The second period was Autumn holiday, where the number of guests was $32 \%$ higher compared to the control week. The chimpanzee behaviour was recorded based on an ethogram constructed by the aid from the zookeepers as well as own observations of the chimpanzees e.g. passive behaviour, movement, foraging, reaction to guests or zookeepers, abnormal behaviour and interaction (Appendix B).

The behaviour of the chimpanzees was collected by using five cameras (ANNOX outdoor action and ycams); three overlooking the outdoor area and two the indoor area. It was not possible to record the area between the outdoor and indoor part of the enclosure, hence chimpanzees that stayed in this area were not recorded. During the control week period 1) the chipanzees were video recorded September 30th, October 2nd and 5th and in during Autumn holiday 2) period the chipanzees were video recorded October 16th, 18th and 20th. Three independent researchers watched the recorded videos separately, discussed discrepancies and guidelines for the behavioural tendencies in order to standardize the ethogram. In addition a correlation test was made in Past to secure that recordings were made in the same way by different observers. While watching the recorded videos the start- and stop points for all behavioural tendencies was noted in Excel. It was converted to seconds after 9.00 AM and afterwards calculated to intervals of each behaviour.

\section{Data Analysis}

All statistical tests were made using the free statistical software program "Past" (Hammer et al., 2001). Behavioral observations were used to: 1) examine if there was an overall difference in how the chimpanzees behave between the control week and the holiday week. 2) to examine if there was an individual difference in the behaviour of the chimpanzees between the control week and the holiday week.

The average time when chimpanzees were "out of view" in all collected data was $21.8 \%$. Prior to the analysis the data were tested for normal distribution with Shapiro-Wilks test (Zar, 1999). As some data were not normally distributed non-parametric tests were used. Level of significans was $<0.05$.

Pairwise comparisons between behavioural recordings in period 1) the control week- and 2) Autumn holiday was made borth for each individual separatly and pooled for all four individuals behavioural. 
Comparisons were tested using Kruskal Wallis and Mann-Whitney pairwise tests (Mann, 2013; MacFarland and Yates, 2016). The correction for multiple comparisons were not corrected because of the reduction in statistical power and the increased possibility for a type two error as the observations were not independent (Nakagawa, 2004). The differences in skewness and kurtosis were checked comparing bootstrap confidence intervals between the groups. To illustrate the differences in behaviour between the chimpanzees, figures were made for the medians, interquartile range, skewness and kurtosis in Excel. Furthermore, cumulative frequency curves were made to estimate how each behaviour was distributed during the day for each chimpanzee (Appendix C).

The percent of the total time consumption of each behaviour for either the control or the holiday was tested for significant differences by a c2 test in Past.The variance was calculated by the interquartile range (IQR); removing data outside the 25th and 75th percentiles, prior to Levene's test.

A shift from one behavior to another is defined as when the chimpanzee stops one type of behavior and begins with another.

\section{Results}

The only behavioural interaction that differed between the individuals in both control week and holiday week was the percentage of time spent with interaction. Jutta spent significantly more time interacting with other individualss than her offspring in the control week and also than Sebastian in the holiday week. Only My spent significantly more time with interaction than Jutta during the holiday week (Appendix D).

\section{Number of visitors did not affect behavior of chimpanzee significantly}

Comparisons of behavioural expressions between the days of control and holiday show no clear tendencies for the medians, IQR, skewness and kurtosis. Although significant individual behavioural differences were found in the data, no overall consensus in significant difference for median, IQR, skewness and kurtosis was found when comparing control days and holidays (Appendix E and F). Hence, the number of visitors is not the main determinant for the significant differences that was observed between control and holiday.

\section{Individual differences in behaviour of the four chimpanzees}

Passive behaviour (Figure 1). My showed significantly lower median passive behaviour than Jutta and Sebastian during the holidays (Figure 1.A). My's and Laura's IQR was significantly lower than that of Sebastian's and Jutta's during the control days. Jutta's IQR was significantly higher than that of the rest of the chimpanzees during the holidays. Laura's IQR was significantly lower than Sebastian's and My's during both control days and holidays (Figure 1.B).

Moving behavior (Figure 2). There is significant difference in the time spendt moving between My and Jutta for both the control days and the holiday. In the control week the median for moving is lowest for Jutta, whereas in the holiday the median for moving is higher than that of My (Figure 2.A). Laura and Jutta's skewness and kurtosis are significantly lower than both My's and Sebastian's skewness and kurtosis for the control (Figure 2.C and 2.D). In the control days the IQR for movents for My is significantly higher than the other chimpanzees. Laura's IQR movements is also significantly lower than Jutta's and Sebastian's IQR for the control. Laura's IQR is significantly lower than the others for the holiday (Figure 2.B).

Fouraging behaviour Figure 3. Jutta's fouraging median is higer than that of the other chimpanzees and significantly higer during the holiday week. The median for Jutta is significantly higher compared to Sebastian's and My's during the control week. My's median fouraging behaviour is significantly lower than Laura's during the control week. (Figure 3.A). My's IQR is also significantly lower than the others in the control week and Laura's and My's IQR significantly lower than Jutta and Sebastian during the holiday week (Figure3.B).

Reaction to guests or zookeepers (Figure 4). Jutta's reacts less to presence of guests and zookeepers (median is significantly lower) than My's in the control week. During the holiday, Sebastian's median is significantly lower than Jutta's (Figure 4.A). Also IQR of Sebastian is significantly higher than Jutta's during the control week. Jutta's IQR is significantly higher than Sebastian's during for the holiday (Figure 4.B). Laura was excluded from figure 4 due to lack of data. 
Stereotypic behaviours (Figure 5). My was less stereotypic than Jutta and Laura (median of My significantly lower than Jutta's and Laura's) both in the control week and during the holidays. Also, My's IQR was significantly lower than the others during the control week. Jutta's IQR is significantly higher than My's in the holiday (Figure 5.B) Sebastian is excluded in stereotypic behaviour due to lack of data.

Behavioural interactions (Figure 6). Laura spent longer time interacting than the other chimpanzees. Laura's median was significantly higher than Sebastian's and My's. During the holiday My's median was significantly lower than Jutta's and Laura's. Laura's median is significantly higher than Sebastian's median (Figure 6.A). Laura's IQR and kurtosis was significantly lower than Sebastian's in the control week (Figure 6.D). Sebastian's IQR was significantly lower than Jutta's and Laura's in the holiday (Figure 6.B).

Laura's number of behavioural shifts were significantly higher than Sebastian's and lower than My's in the control week. In addition, Laura's number of behavioural shifts were significantly higher than Jutta's and Sebastian's in the holiday. My's number of behavioural shifts was significantly higher than the others during the holiday (Appendix J).

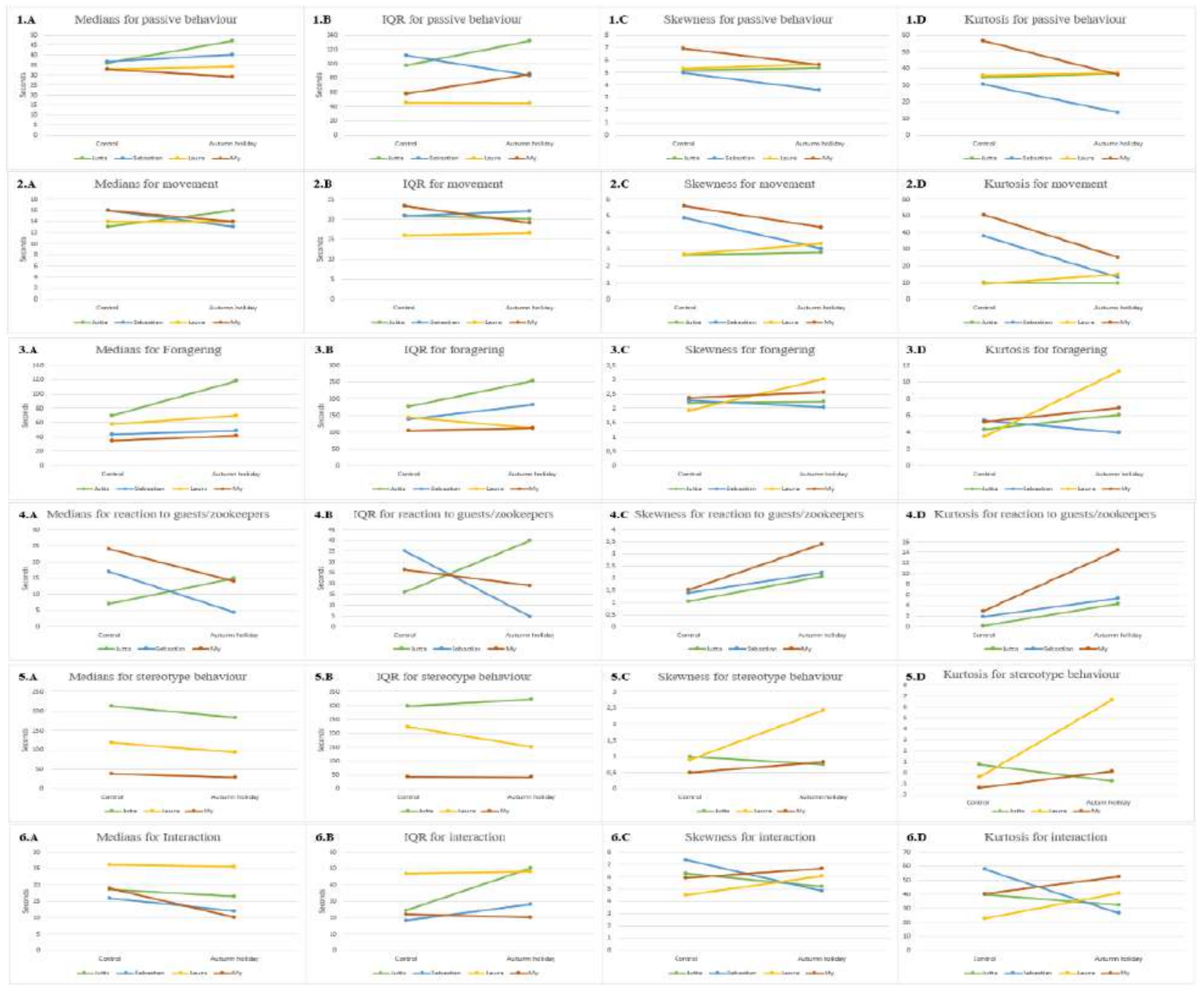

Figure 1-6 show the different behaviours; 1) passive behaviour, 2) movement, 3) foraging, 4) reaction to guests/zookeepers, 5) stereotype behaviour and 6) interaction.

All the figures show a) medians, $b$ ) interquartile range, $c$ ) skewness and d) kurtosis for the behaviour in the control week and the autumn holiday for the four chimpanzees (For further details see Appendix G and H). 


\section{Discussion}

\section{No changet in Behaviour As A result Of An Increase In Visitors}

Even though there were $32 \%$ increase in the number of visitors during the holiday week this study found no clear patterns indicating that the number of zoo visitors had an effect on the behaviour of the chimpanzees. Several studies found that zoo visitor's presence, density, noise and activity have an influence on animal behaviour (Davey, 2007; Fernandez et al., 2009; De Azevedo 2012; Quadros et al., 2014). Larsen et al. 2014 found that noise influenced koalas making them more vigilance and Mitchell et al. (1992) found that if there was a large active audience primate's behaviour were directed at them.

\section{Individual Variation In Behaviour Of The Four Chimpanzees}

One of the most notable results found was that My generally performed significantly more shifts in behaviour i.e shorter behaviour intervals (Appendix J) and Jutta's higher percent interactions compared to her offspring (Appendix D). This may be due to age, individual behavioural trends and Jutta's role as a mother. When chimpanzees reach adolescence, spontaneous behaviour tend to decrease (Schrier and Stillnitz, 1974). Another reason could be her young age because play frequency decreases from puberty and is approximately proportional to increasingly longer and more intense grooming sessions (Van Lawick-Goodall, 1968).

Jutta's more interactive behavioural may be due to her role as a mother and her higher rank. Female primates with high rank has previously been shown to receiving more grooming than others (Seyfarth, 1977; Tiddi et al. 2012). Jutta's significantly long foraging time may be explained by ther older age. Logan and Sanson (2002) found that koalas (Phascolarctos cinereus) compensate for tooth wear by increased food intake (enhanced average number of consumed leaves, daily chews and feeding time) and King et al. (2005) found that sifakas with tooth wear had a poorer reduction in particle size in dentally sentence compared to conspecifics in dental prime.

Although, Birkett and Fisher-Newton (2011) found that variation in stereotypic behaviour could not be explained by age, My's significantly less stereotypic behaviour, may be explained by her young age. In our study there was tendency for the youngest chimpanzee being less stereotypic than the older chimpanzees. Stereotypic behaviour may be caused by boredom, restlessness and fatigue contray to more spontaneous and playfull behaviour in young chimpanzees. However, stereotypic behaviour may also be related to differences in personalities. Maternal separation cause stereotypical behaviour (Birkett and FisherNewton 2011). Jutta was transferred to Aalborg zoo when she was only six years old and may have been separated from her mother in a young age. As Jutta was born in Africa, in a different environment, while the other three chimpanzees are born in Aalborg zoo, may also have affected Jutta's behaviour.

\section{Conclusion}

This study revaled no significant differences in the behaviour of the chimpanzees related to an increase in the number of visitors in Aalborg Zoo during Autumn holiday. Individual behavioural differences were found in interaction behaviour, fouraging behaviour and stereotypic behaviour. This may be explained by the age or individual behavioural trends in the four chimpanzees.

\section{Acknowledgments}

The authors want to thank the staff at Aalborg Zoo, with special thanks to the staff at the primate department for their time and participation in this study. Also, thanks to Cino Pertoldi for guidance in writing this paper.

\section{Author's Contrinutions}

All authors contributed equally.

\section{References}

AZA Ape TAG 2010. Chimpanzee (Pan troglodytes) Care Manual, Association of Zoos and Aquariums, Silver Spring, MD.

Boesch C. 1996. Social grouping in Tai chimpanzees in McGrew WC, Merchant LF, Nashida T. (eds.), Great Ape Societies, Cambridge: Cambridge University Press, pp. 103-113. 
Birkett LP. Newton-Fisher N. E. 2011. How Abnormal Is the Behaviour of Captive, ZooLiving Chimpanzees?, Plos One 6: 1-7, doi: https://doi.org/10.1371/journal.pone.0020101.

Carere C. Caramaschi D. Fawcett TW 2010. Covariation between personalities and individual differences in coping with stress: Converging evidence and hypotheses, Current Zoology 56 (6): 728-740.

Carder G. Semple S 2008. Visitor effects on anxiety in two captive groups of western lowland gorillas, Animal Behaviour Science 115: 211-220

Clarke AS. Juno C.J. Maple TL 1982. Behavioral Effects of a Change in the Physical Environment: A Pilot Study of Captive Chimpanzees, Zoo Biology 1: 371-380

Clubb R. Mason GJ 2006. Natural behavioural biology as a risk factor in carnivore welfare: How analysing species differences could help zoos improve enclosures, Elsevier B. V., doi: 10.1016/j.applanim.2006.05.033.

Cronin KA. West V. Ross SR 2016. Investigating the relationship between welfare and rearing young incaptive chimpanzees (Pan troglodytes), Applied Animal Behaviour Science 181 pp. 166-172, doi: 10.1016/j.applanim.2016.05.014.

Davey G 2007. Visitors' Effects on the Welfare of Animals in the Zoo: A Review, Applied Animal Welfare Science 10(2): 169-183, doi: 10.1080/10888700701313595.

De Azevedo CS. Lima MF F. Da Silva VCA. Young RJ. Rodrigues M 2012. Visitor Influence on the Behavior of Captive Greater Rheas (Rhea americana, Rheidae Aves), Journal of Applied Animal Welfare Science15(2): 113-125, doi: 10.1080/10888705.2012.624895.

De Waal FBM 2005. A century of getting to know the chimpanzees, Nature 437: 56-59.

Dingemanse NJ. Reale D 2005. Natural Selection and Animal Personality. Behaviour 142 (9-10): 11591184, doi: 10.1163/156853905774539445.

Fernandez EJ. Tamborski MA. Pickens SR. Timberlake W 2009. Animal-visitor interactions in the modern zoo: Conflicts and interventions, Applied Animal Behaviour Science 120: 1-8, doi: 10.1016/j.applanim.2009.06.002.

García JOP. Miani A. Alstrup AKO. Malmkvist J. Pertoldi C. Jensen TH. Nielsen RK. Hansen DW. Bach LA 2020. Orangulas: effect of scheduled visual enrichment on behavioral and endocrine aspects of a captive orangutan (Pongo pygmaeus). Journal of Zoo and Aquarium Research 8: 67-72. https://doi.org/10.19227/jzar.v8i1.416.

Hammer Ø. Harper DAT. Ryan PD 2001: PAST: Paleontological statistics software package for education and data analysis. Palaeontologia Electronica 4(1) 9, http://palaeo-electronica.org/2001_1/past /issue 1_01.h tm [12.12.2019]

Hosey GR 2005. How does the zoo environment affect the behaviour of captive primates?, Elsevier 90(2): 107-129, doi:10.1016/j.applanim.2004.08.015

Hosey G. Druck P 1987. The influence of zoo visitors on the behavior of captive primates, Applied Animal Behavior Science18: 19-29.

King SJ. Arrigo-Nelson SJ. Pochron ST. Semprebon GM. Godfrey LR. Wright PC. Jernvall J. 2005. Dental senescence in a long-lived primate links infant survival to rainfall, The National Academy of Science of the USA102 (46):16579-16583, doi: 10.1073pnas.0508377102.

Kreger MD. Mench JA. 1995. Visitor-Animal Interactions At the Zoo in Anthrozoös 8(3): 143-158.

Kühl H. S. Sop T. Williamson E. A. Mundry R. Brugière D. Campbell G. Cohen H. Danquah E. Ginn L. Herbinger I. Jones S. Junker J. Kormos R. Kouakou C. Y. N'Goran P. K. Normand E. ShuttPhillips K. Tickle A. Vendras E. Welsh A. Wessling EG. Boesch C 2017. The Critically Endangered western chimpanzee declines by 80\%, American Journal of Primatology 79 (9), doi: 10.1002/ajp.22681.

Larsen MJ. Sherwen SL. Rault J 2014. Number of nearby visitors and noise level affect vigilance in captive koalas in Applied Animal Behaviour Science 156: 76-82, doi: 10.1016/j.applanim.2014.02.005

Lecturer ATS. Booth R. 2013. Assessing the Role of Zoos in Wildlife Conservation in Human Domensions of Wildlife, vol. 8, iss. 1, pp. 65-74, doi: 10.1080/10871200390180163

Logan M. Sanson GD 2002. The effect of tooth wear on the feeding behaviour of free-ranging koalas (Phascolarctos cinereus, Goldfuss), The Zoological Society of London 256, pp. 6369, doi: 10.1017/S0952836902000080

MacFarland TW. Yates JM 2016. Introduction to Nonparametric Statistics for the Biological Sciences Using R, Springer Nature, pp. 47-48, ISBN: 978-3-319-30634-6 
Mann S 2013. Introductory Statistics - international student version, 8th edition, John Wiley \& Sons Singapore, chapter 12 and 15, ISBN: 978-1-118-31870-6

Martínez RC 2014. Use of natural resources by sanctuary chimpanzees (Pan troglodytes): understanding the significance of naturalistic environments, Master in Applied Ecology, Hedmark University College

Mitchell G. Tromborg CT. Kaufman J. Bargabus S. Simoni R. Geissler V 1992. More on the 'influence' of zoo visitors on the behaviour of captive primates, Applied Animal Behaviour Science, vol. 35, iss. 2, pp. 189-198, doi: 10.1016/0168-1591(92)90009-Z

Penfold LM. Powell D. Traylor-Holzer K. Asa CS 2013. "Use it or Lose it": Characterization, Implications, and Mitigation of Female Infertility in Captive Wildlife, Wiley Periodicals, Inc., doi: 10.1002/zoo.21104

Pomerantz O. Meiri S. Terkel J 2013. Socio-ecological factors correlate with levels of stereotypic behaviour in zoo-housed primates, Behavioural Processes 98 pp. 85-91, doi: 10.1016/j.beproc.2013.05.005

Pruetz JDE. McGrew WC 2001. What does a chimpanzee need? Using natural behaviour to guide the care and management of captive populations. In: Brent L. (ed.), The Care and Management of Captive Chimpanzees. San Antonio, Texas: The American Society of Primatologists, pp. 17-37

Quadros S. Goulart VDL. Passos L. Vecci MAM. Young RJ. 2014. Zoo visitor effect on mammal behaviour: Does noise matter?, Applied Animal Behaviour Science, vol. 156, pp. 78-84

Ralls K.Ballou JD 1992. Managing Genetic Diversity in Captive Breeding and Reintroduction Programs, Transactions of the 57th North American Wildlife and Natural Resources Conference, Wildlife Management Institute.

Ross SR. Wagner KE. Schapiro SJ. Hau J. Lukas KE 2011. Transfer and Acclimatization Effects on the Behavior of Two Species of African Great Ape (Pan troglodytes and Gorilla gorilla gorilla) Moved to a Novel and Naturalistic Zoo Environment, Int J Primatol, vol. 32, pp. 99-117, doi: 10.1007/s10764-0109441-3

Schrier AM. Stollnitz F 1974. Behavior of Nonhuman Primates; Modern Research Trends, vol. 5, Academic Press, Inc., USA, pp. 451.

Seyfarth RM 1977. A model of Social Grooming Among Adult Female Monkeys, J. Theor. Biol. 65: 671698.

Standford CB 1998. The Social Behavior of Chimpanzees and Bonobos, Current Anthropology 39 (4): 399420

Skibins JC.Powell B 2013. Conservation Caring: Measuring the Influence of Zoo Visitors' Connection to Wildlife on Pro-Conservation Behaviors in Zoo Biology 32: 528-540.

Thompson V 1976. Observation of the great apes in a naturalistic zoo environment, Chicago: Lincoln Park Zoo, Unpublished manuscript.

Tiddi B. Aureli F. Schino G 2012. Grooming Up the Hierarchy: The Exchange of Grooming and RankRelated Benefits in a New World Primate, PLoS ONE 7 (5), doi: 10.1371/journal.pone.0036641

Van Lawick-Goodall J. 1968. The Behaviour of Free-living Chimpanzees in the Gombe Stream Reserve 1(3) 161-311, doi: 10.1016/S0066-1856(68)80003-2

Vestergaard KS. Skadhauge E. Lawson L. G 1997. The Stress of Not Being Able to Perform Dustbathing in Laying Hens, Physiology \& Behaviour, 62 (2): 413-419.

Webb SJN. Hau J.Schapiro SJ 2018 Captive chimpanzee (Pan troglodytes) behaviour as a function of space per animal and enclosure type, American Journal of Primatology, doi: 10.1002/ajp.22749

Wolf M. and Weissing F. J. 2012. Animal personalities: consequences for ecology and evolution, Trends in Ecology and Evolution 27 (8): 452-461.

Wood W 1998. Interactions Among Environmental Enrichment, Viewing Crowds, and Zoo Chimpanzees (Pan troglodytes), Zoo Biology 17: 211-230

Zar JH 1999. Biostatistical Analysis, 4th Edition, Prentice Hall, Upper Saddle River 


\section{Appendix A}

Chimpanzee enclosure

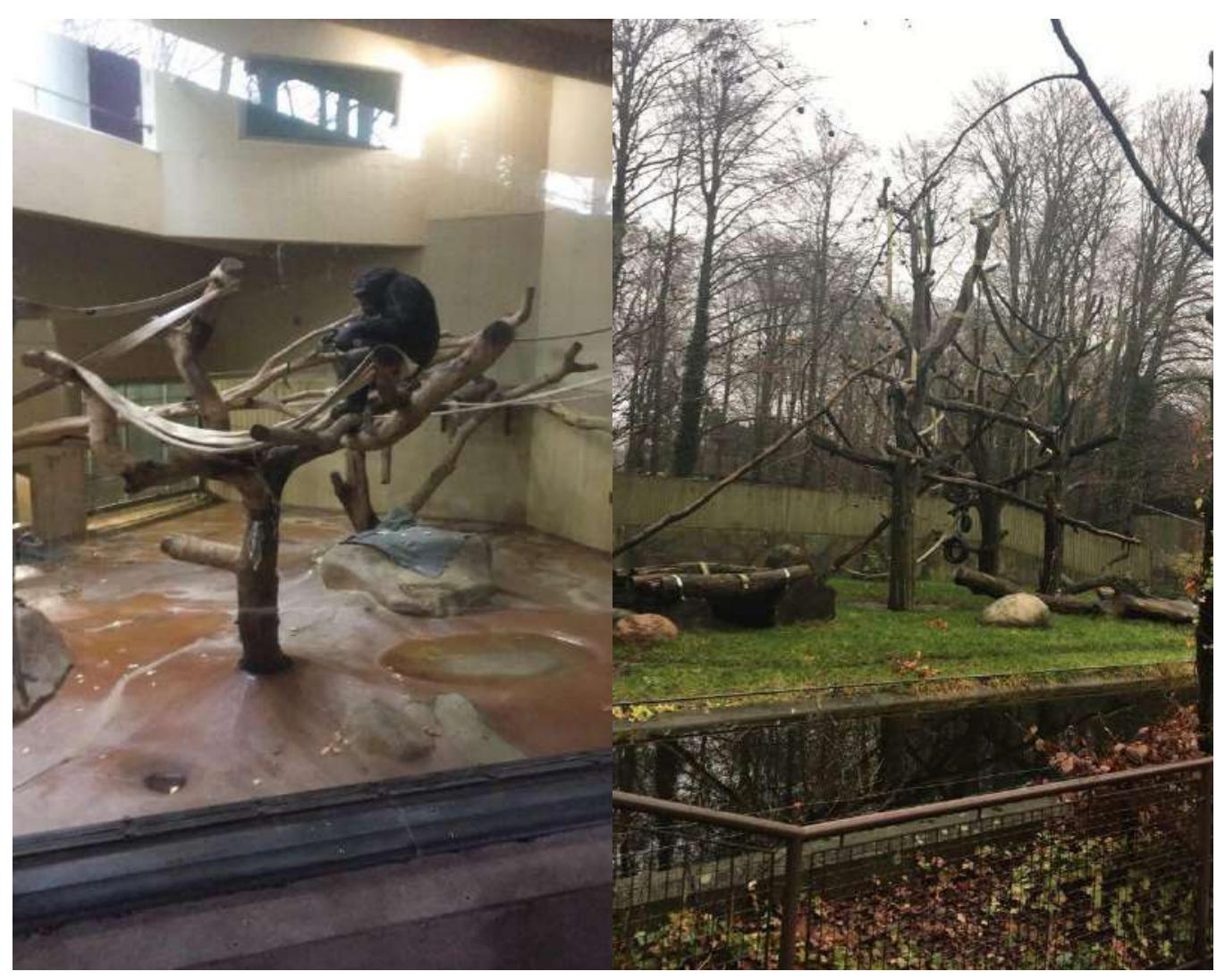


Ottosen et al, 2020. Genet. Biodiv. J, Special issue (Behavioural Instability), 1-60

\section{Appendix B}

Ethogram

Passive behaviour

Sleep, rest, limited movement.

It is okay for the chimpanzee to sit and handle objects, to stand up and look around and take two steps maximum, if they afterwards is passive again.

If they are partly 'out of view', but most of chimpanzee is visible it is okay to note the behaviour as 'passive behaviour', as well if it sits with the back towards the camera, but it is clear that the chimpanzee do not perform any other behaviours.

\begin{tabular}{l|l}
\hline Movement & $\begin{array}{l}\text { Movement, walking, running (with or without objects), swinging in the tireswing, playing } \\
\text { alone and climbing trees or rocks. } \\
\text { It is okay for the chimpanzee to perform 'passive behaviour' if it do not exceed 10 seconds, } \\
\text { if they afterwards moves again. }\end{array}$ \\
\hline Foraging & $\begin{array}{l}\text { Food intake, drinking, putting stick into the container with holes to eat the content. } \\
\text { The behaviour 'foraging' starts at the point the chimpanzee touch the food item. } \\
\text { It is okay for the chimpanzee to move from one food item to another and move while } \\
\text { eating (foraging is considered higher ranking than movement). }\end{array}$ \\
\hline $\begin{array}{l}\text { Reaction to } \\
\text { guests/zookeepers }\end{array}$ & Activity related to guests or zookeepers, banging on rocks or the walls. \\
\hline Stereotype behaviour & Coprophagy, lubricating feces out on objects \\
\hline Interaction & $\begin{array}{l}\text { Not necessarily positive interaction between individuals, grooming, breeding behaviour, } \\
\text { greetings, dominant/submissive behaviour }\end{array}$ \\
\hline
\end{tabular}


Ottosen et al, 2020. Genet. Biodiv. $J$, Special issue (Behavioural Instability), 1-60

\section{Appendix C}

\section{Cumulative curves}

Not enough data for the following: Reaction to guests/zookeepers $(30.09,02.10,16.10,18.10)$ and Stereotype behaviour (30.09, $02.10,16.10,18.10)$.

30.9
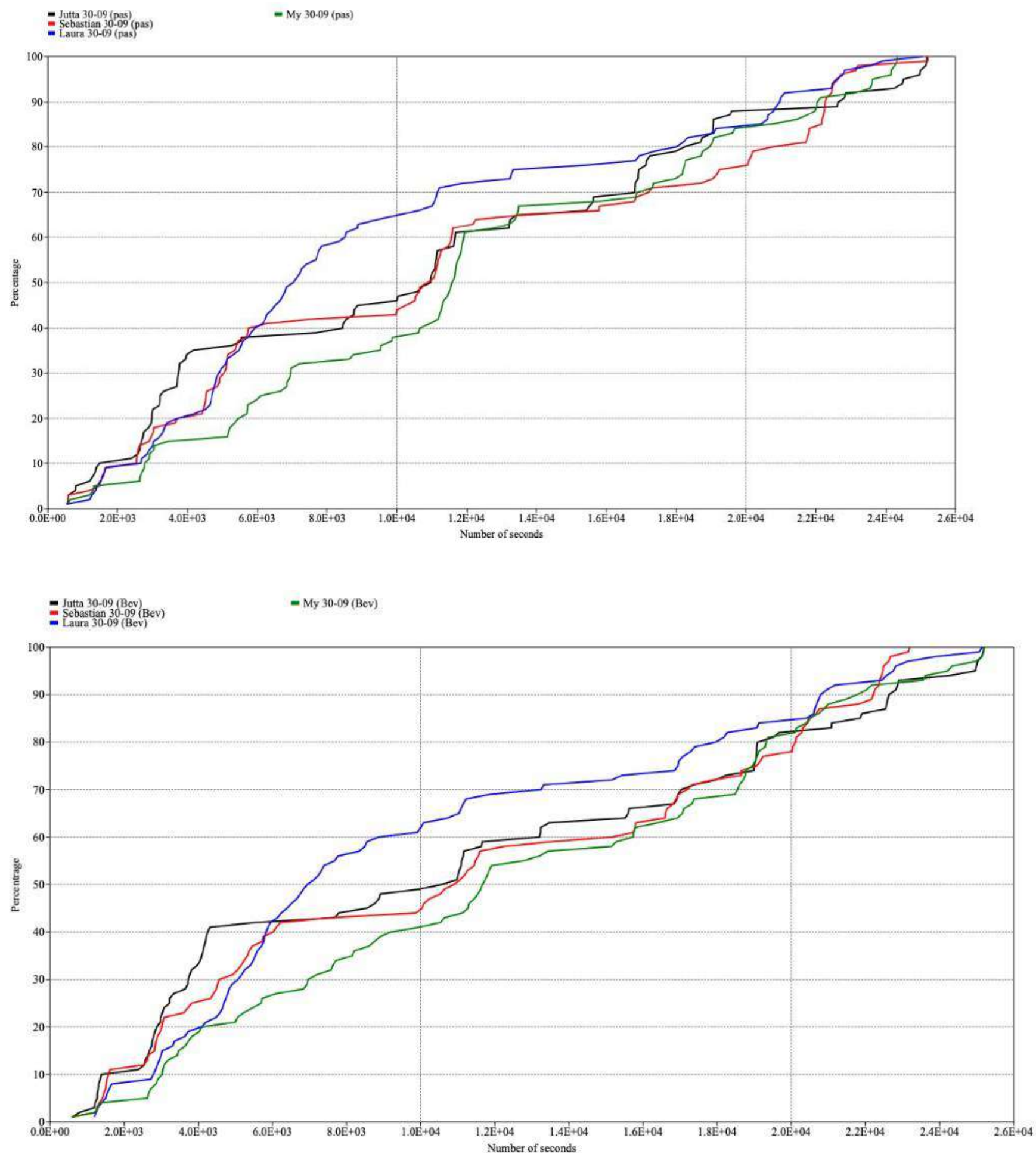
Ottosen et al, 2020. Genet. Biodiv. J, Special issue (Behavioural Instability), 1-60

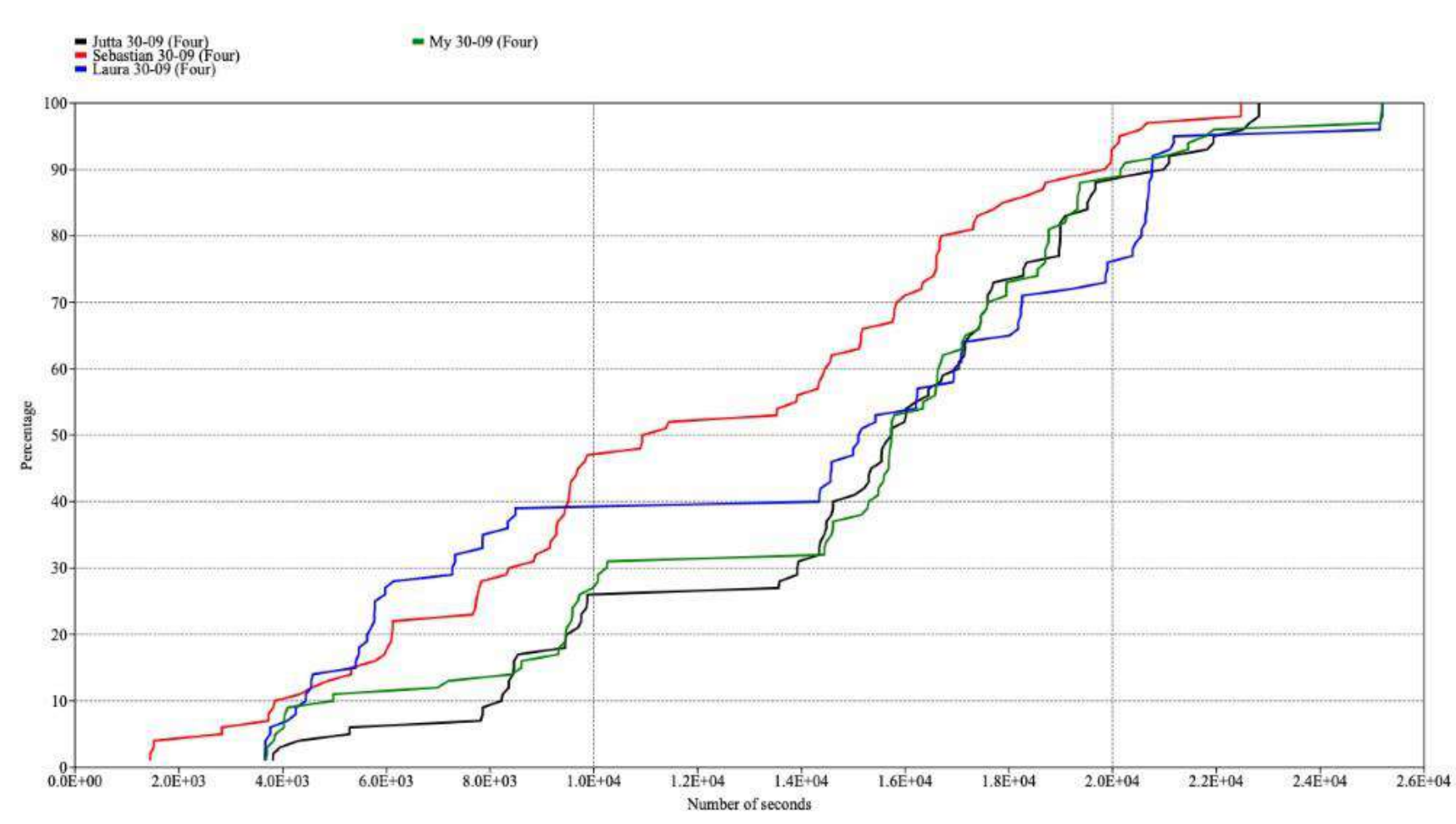

EJutta $30-09$ (Inter.)
= Sebastian $30-09$ (Inter.)
Laura $30-09$ (Inter.)

- My 30-09 (Inter)

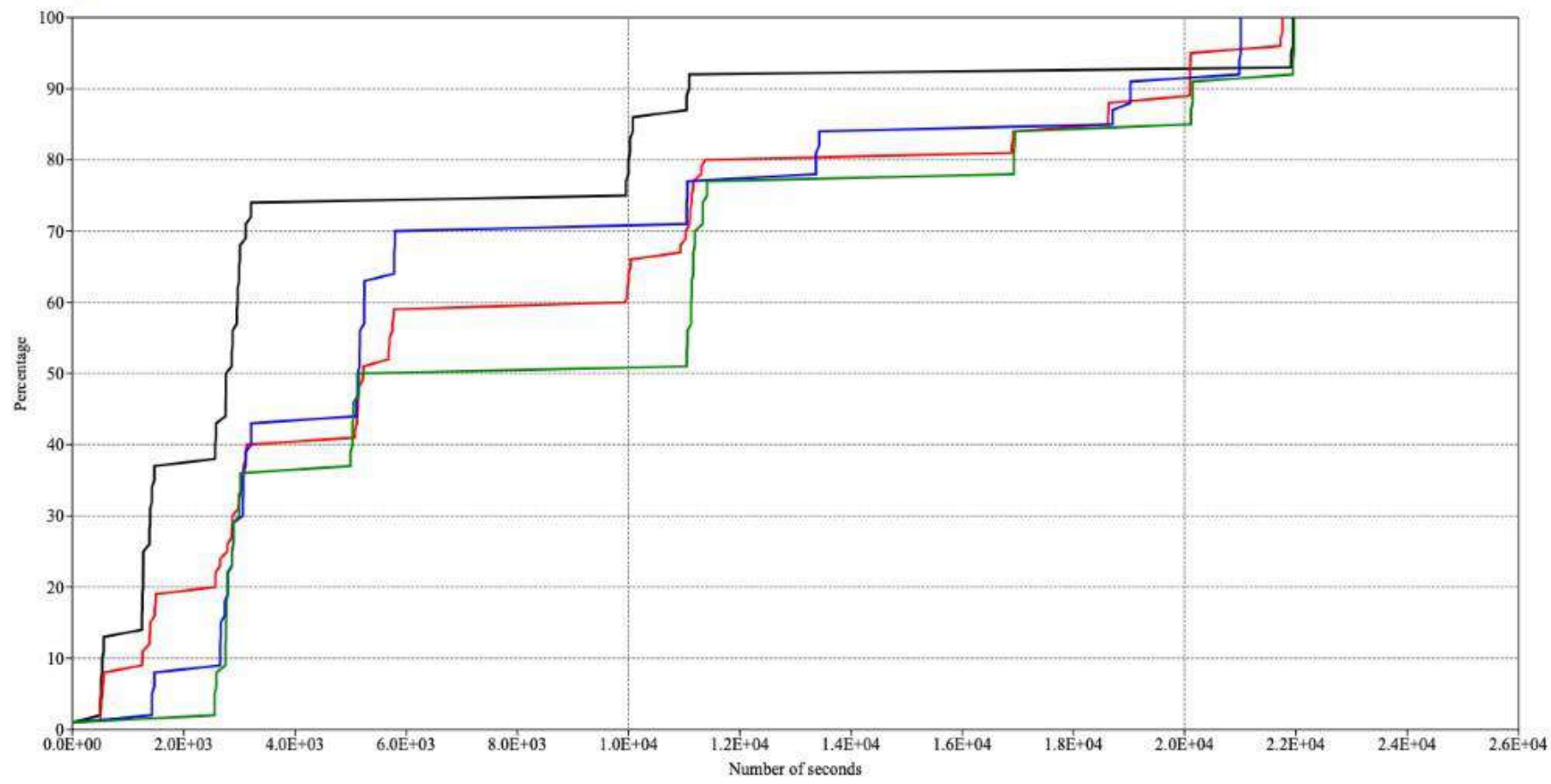


Ottosen et al, 2020. Genet. Biodiv. $J$, Special issue (Behavioural Instability), 1-60

02.10

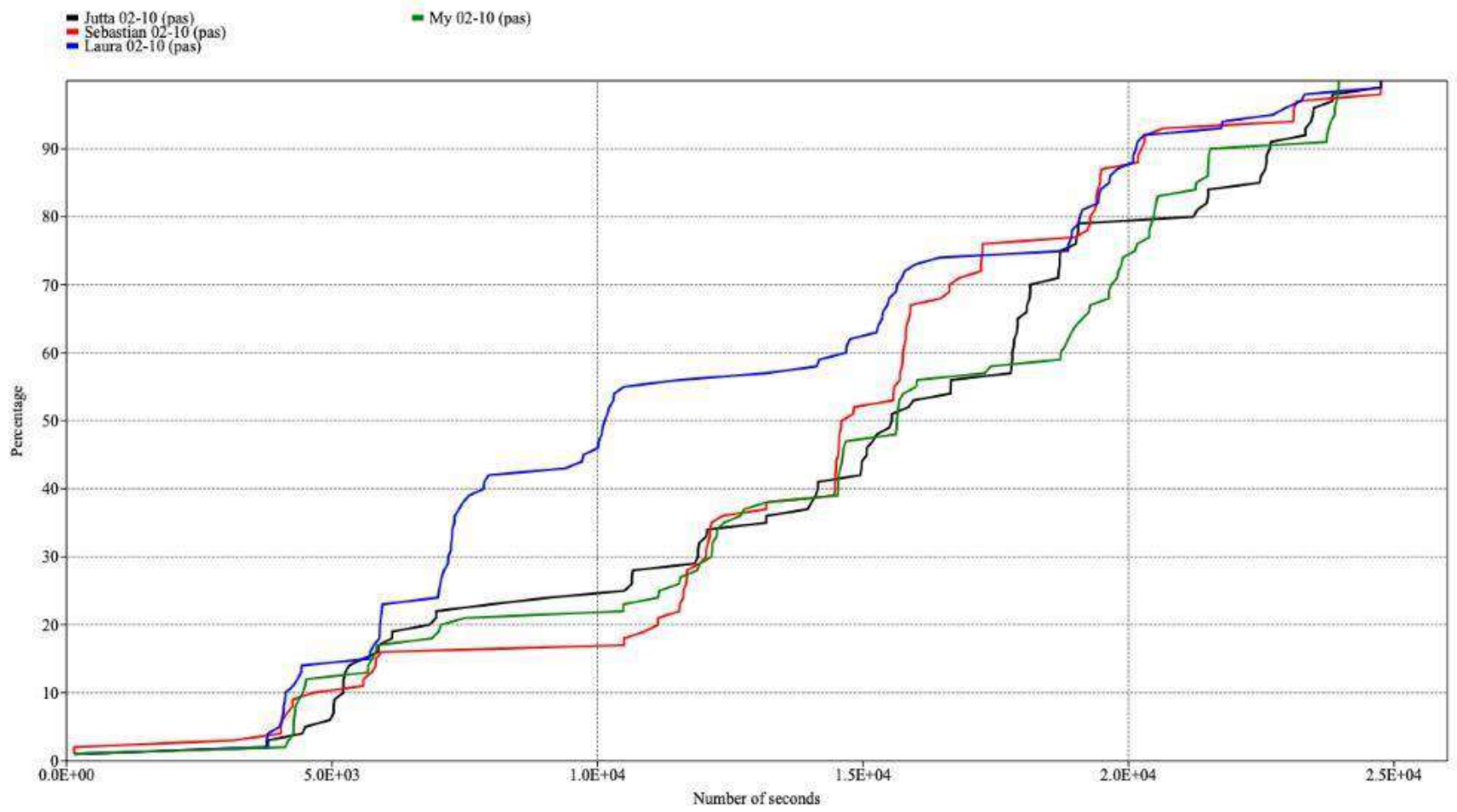

- Jutta $02-10(\mathrm{Bev})$
$=$ - Laura 02-10 (Bev)

- My $02-10$ (Bev)

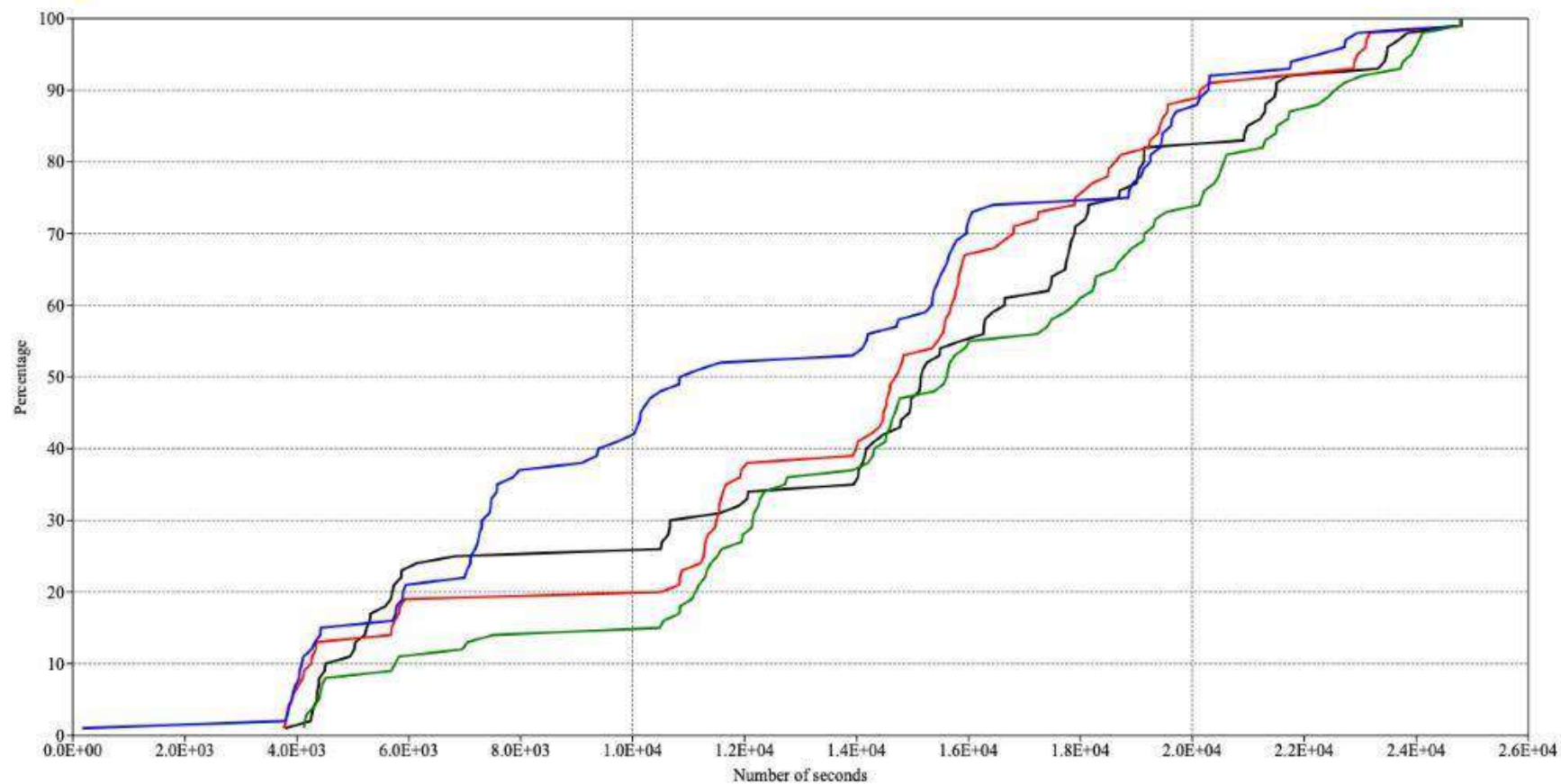


Ottosen et al, 2020. Genet. Biodiv. J, Special issue (Behavioural Instability), 1-60

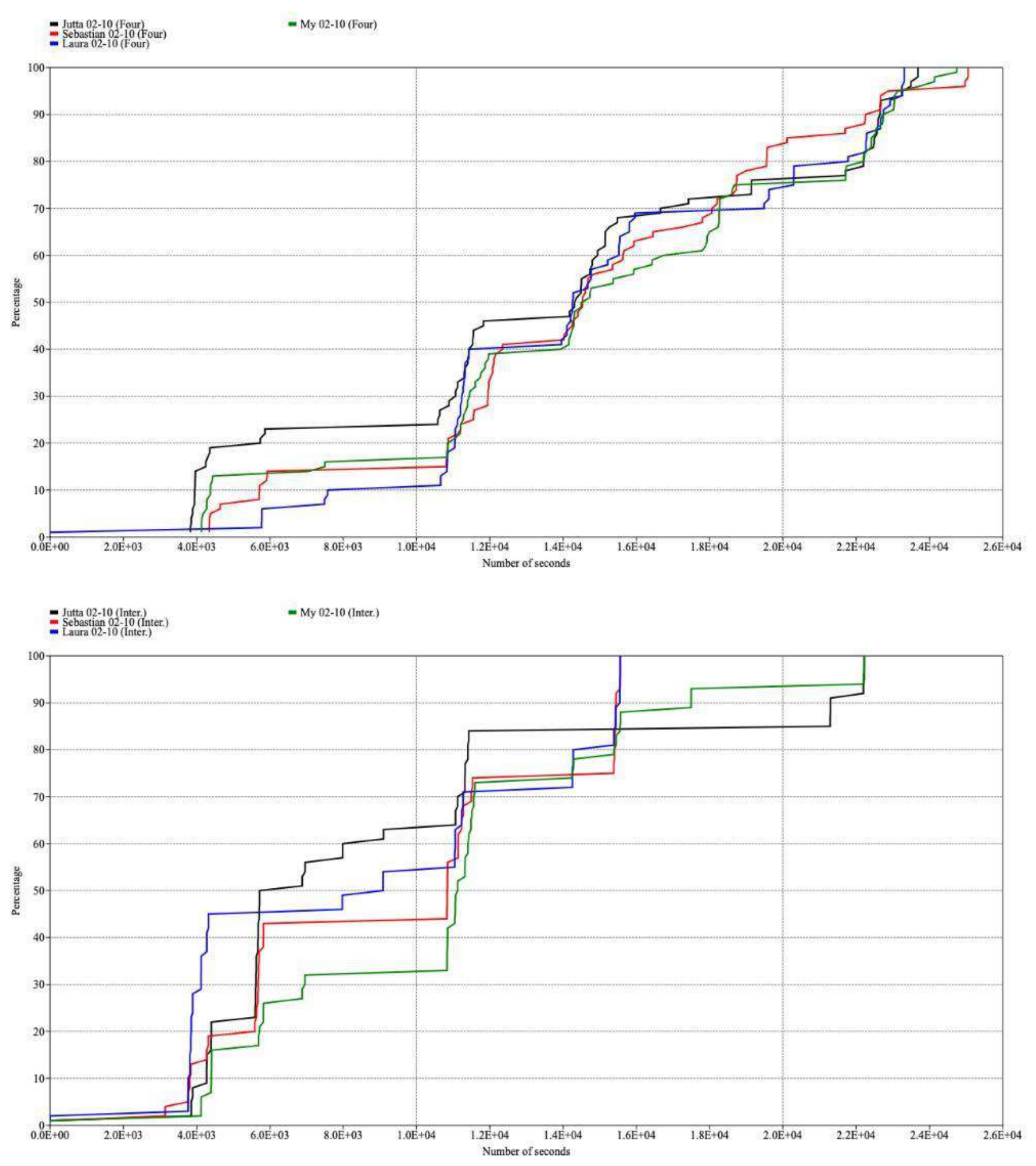


Ottosen et al, 2020. Genet. Biodiv. $J$, Special issue (Behavioural Instability), 1-60

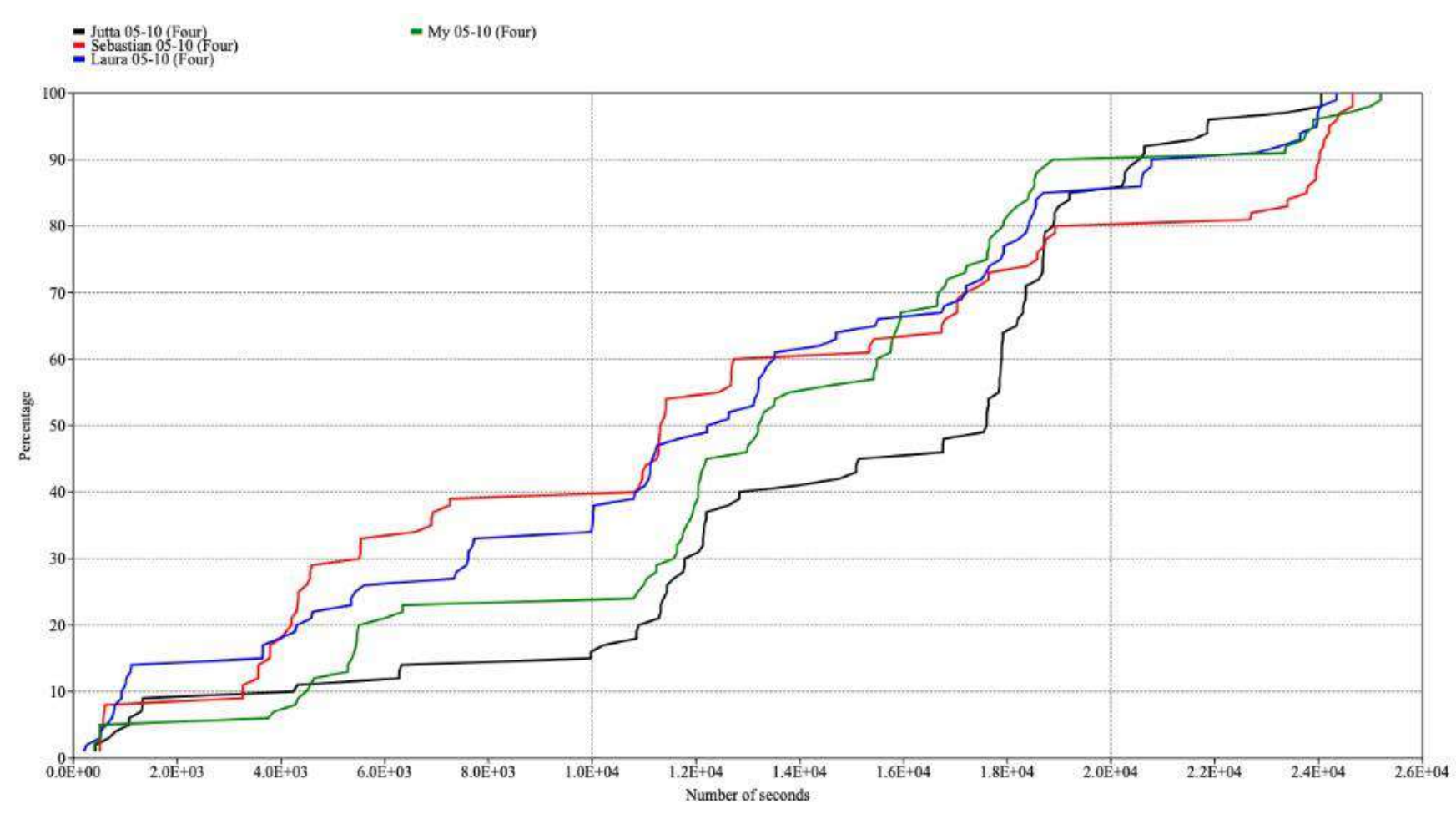

05.10

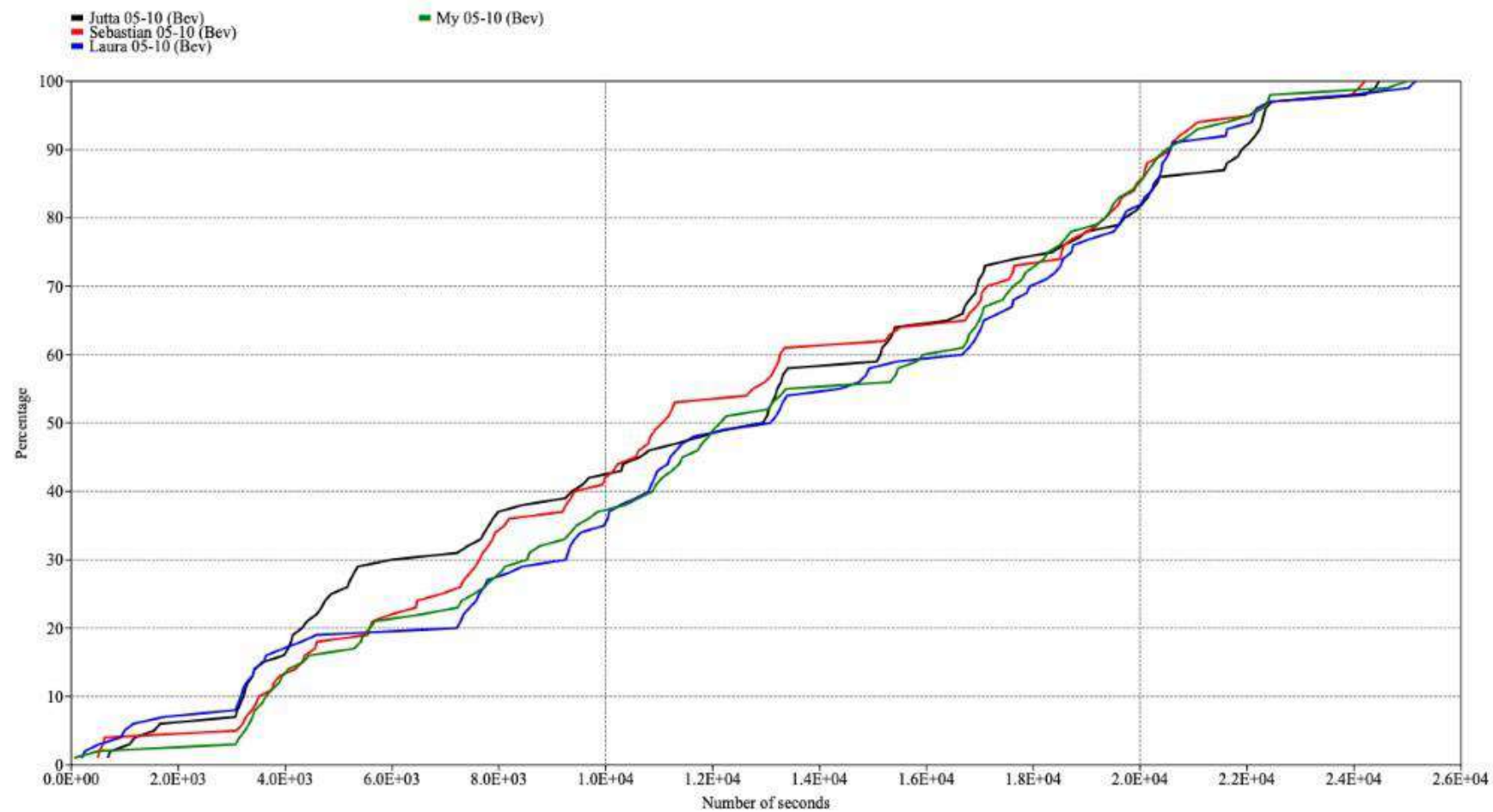


Ottosen et al, 2020. Genet. Biodiv. $J$, Special issue (Behavioural Instability), 1-60

Iutta $05-10$ (Reak.)
My 05-10 (Reak.)

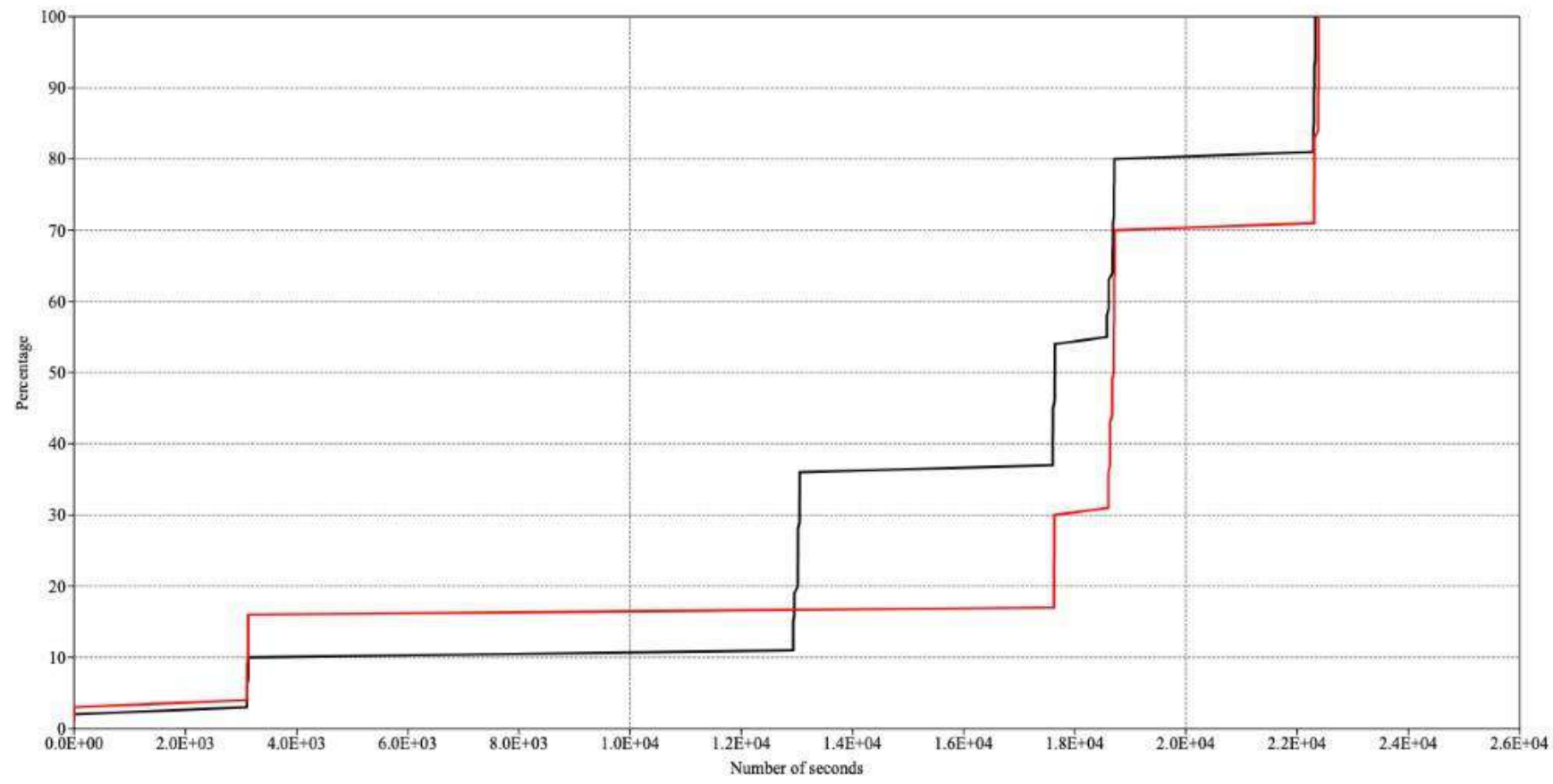

- Jutta $05-10$ (Inter.)

$=$ My $05-10$ (Inter.)

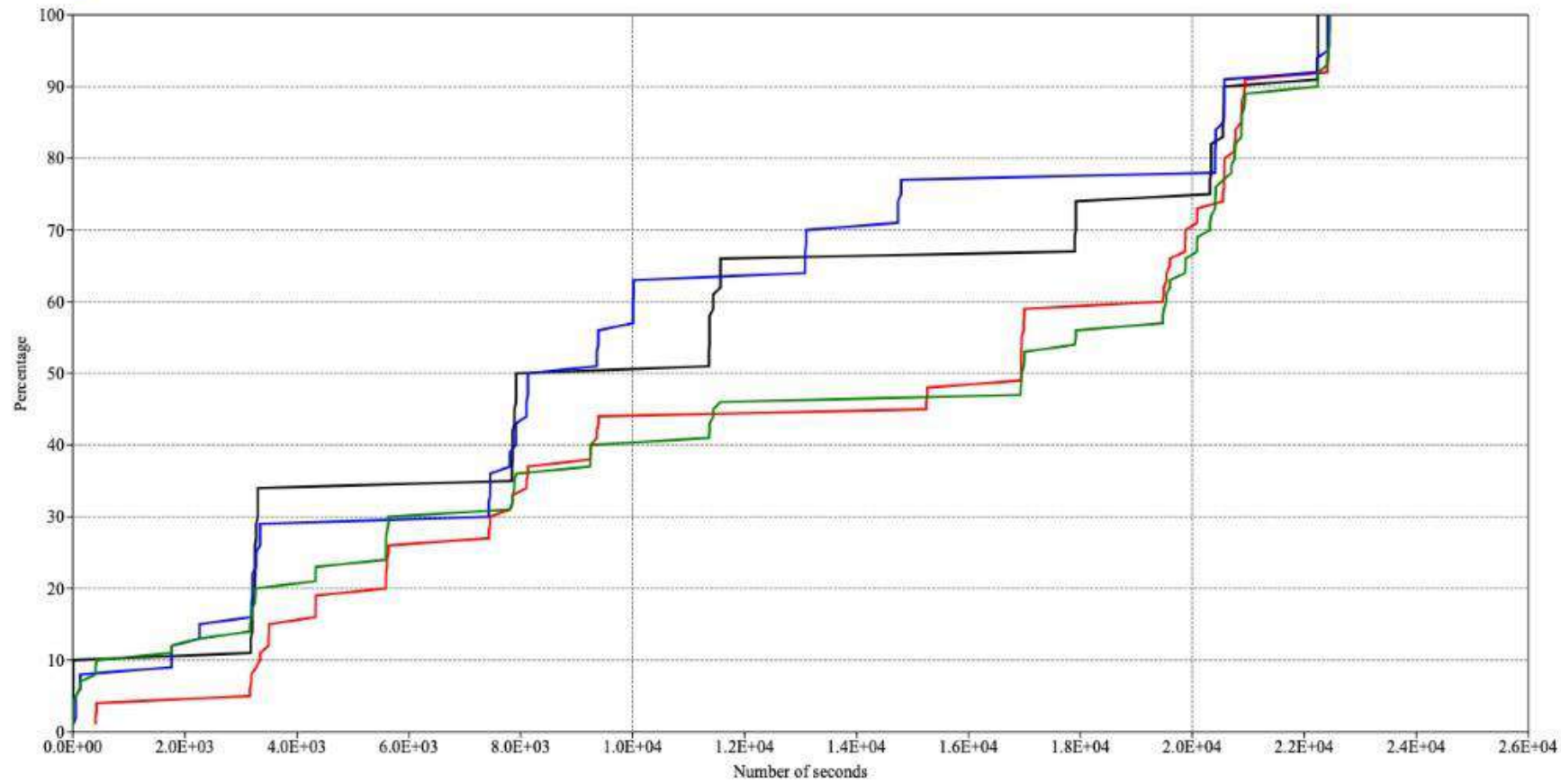


Ottosen et al, 2020. Genet. Biodiv. J, Special issue (Behavioural Instability), 1-60

16.10

- Jutta $16-10(\mathrm{Bev})$
- Sebastian $16-10(\mathrm{Bev})$

$=$ My $16-10$ (Bev)

- Laura 16-10(Bev)

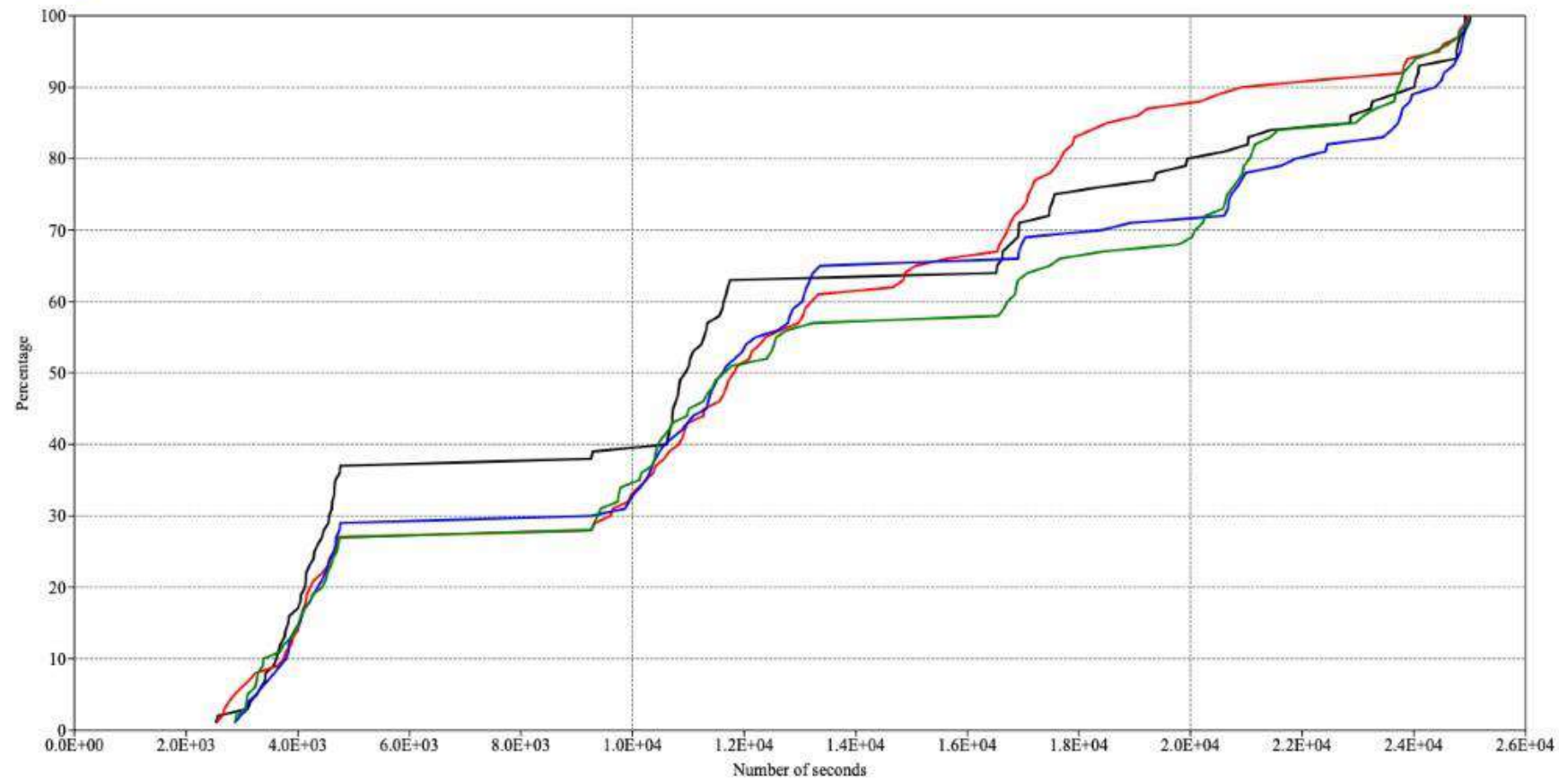

- Jutta 16-10 (pas)

$=$ My $16-10$ (pas)

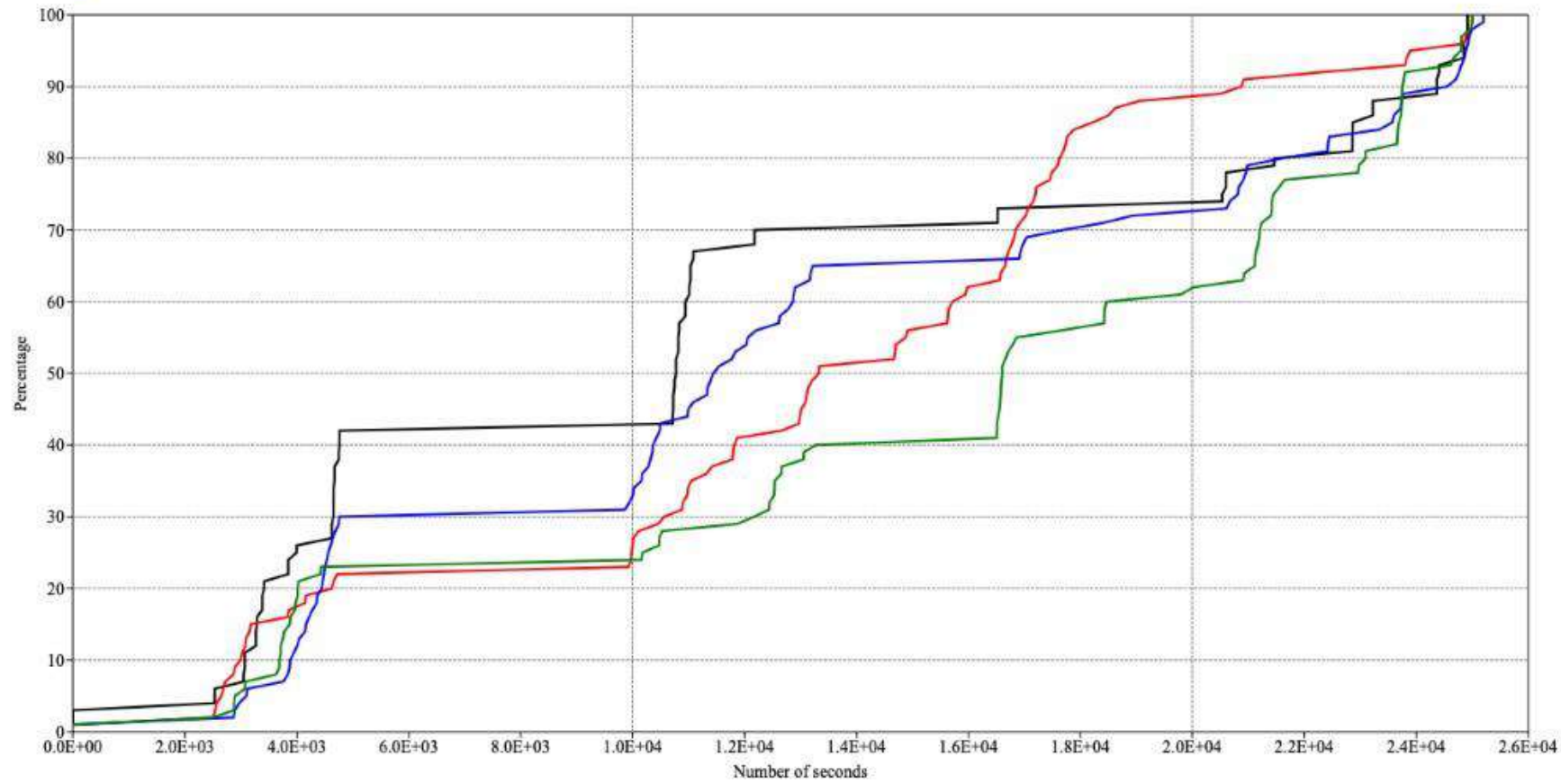


Ottosen et al, 2020. Genet. Biodiv. $J$, Special issue (Behavioural Instability), 1-60
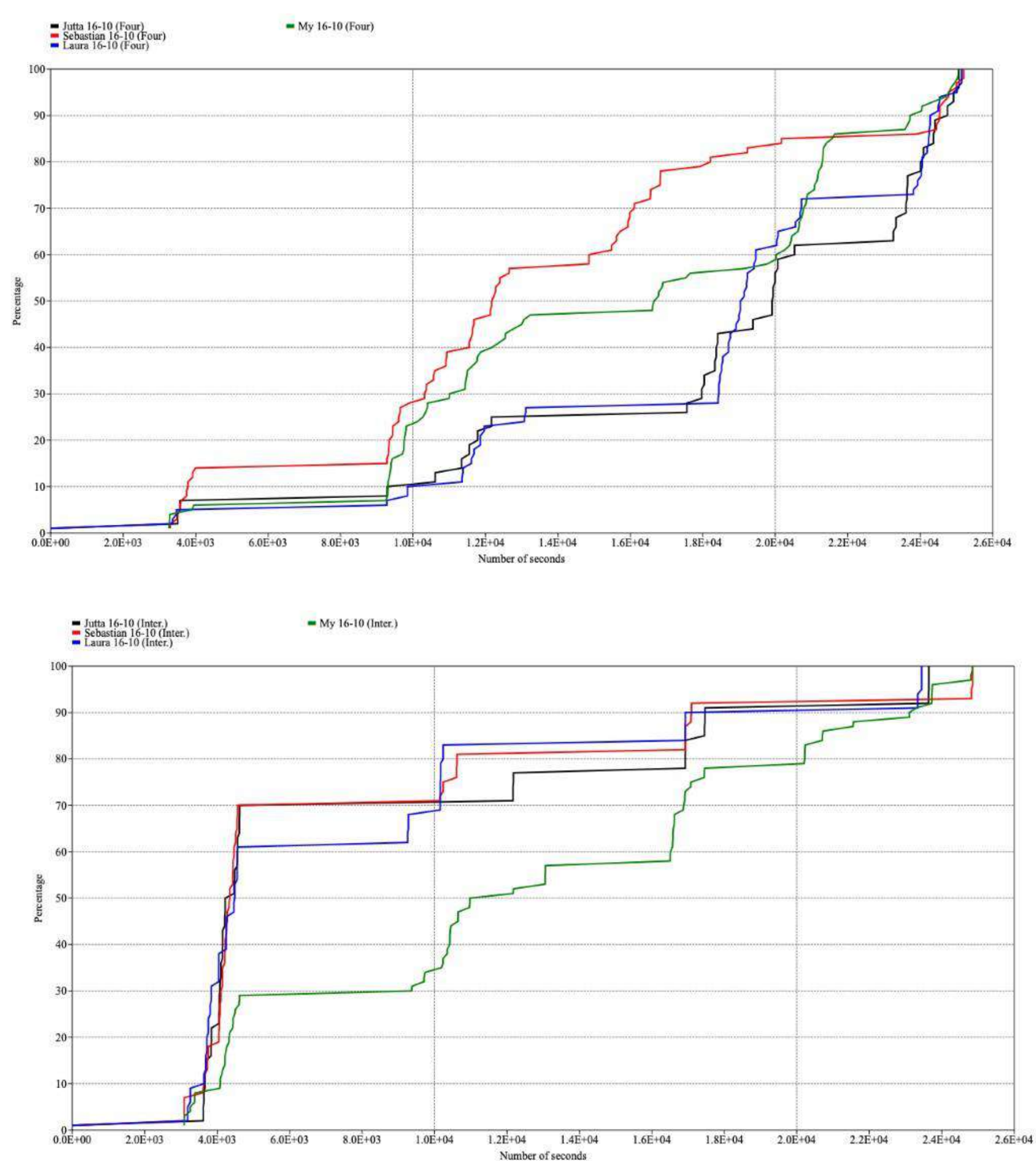
Ottosen et al, 2020. Genet. Biodiv. J, Special issue (Behavioural Instability), 1-60

18.10

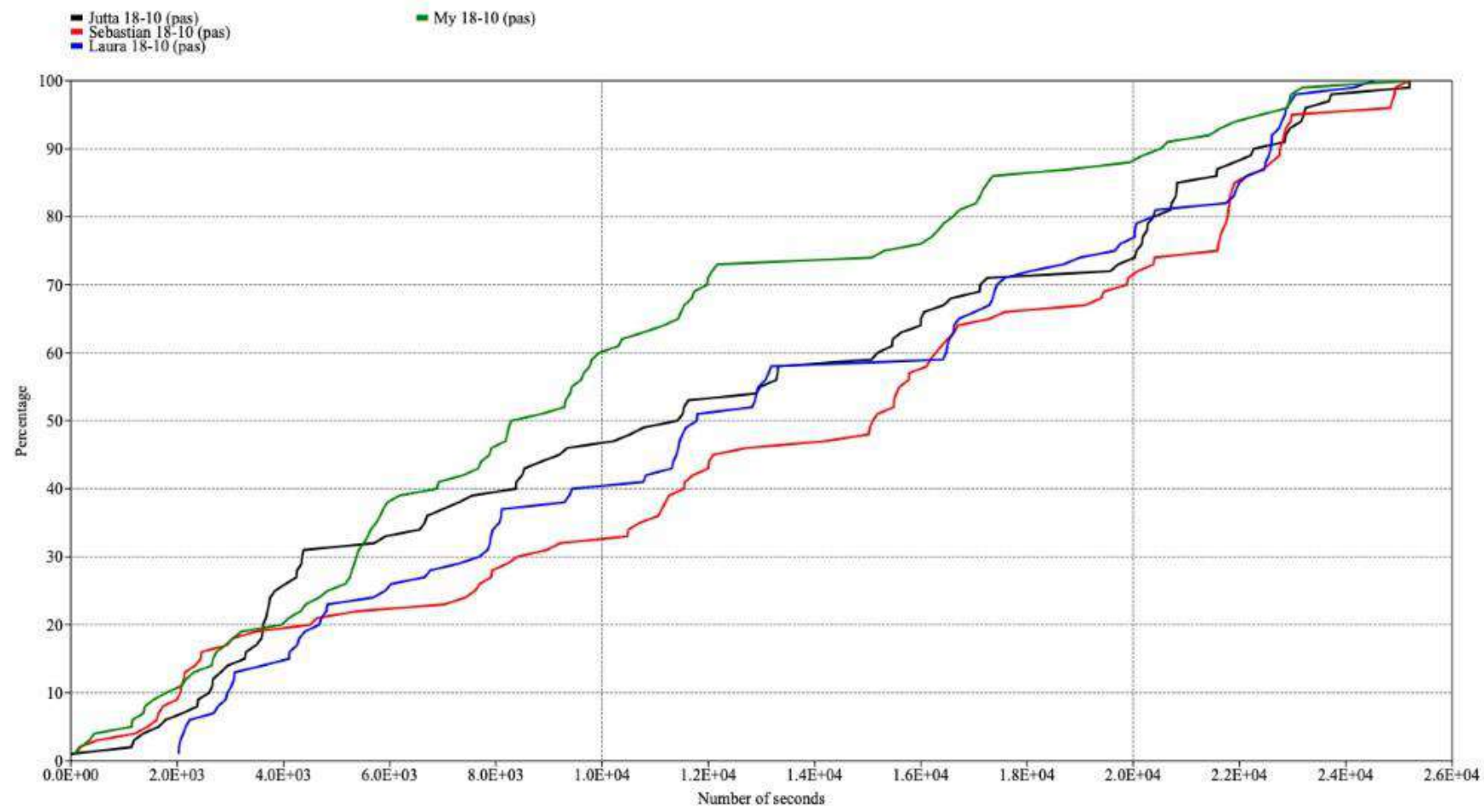

Dutta $18-10(\mathrm{Bev})$
Sebastian $18-10(\mathrm{Bev})$

- My 18-10 (Bev)

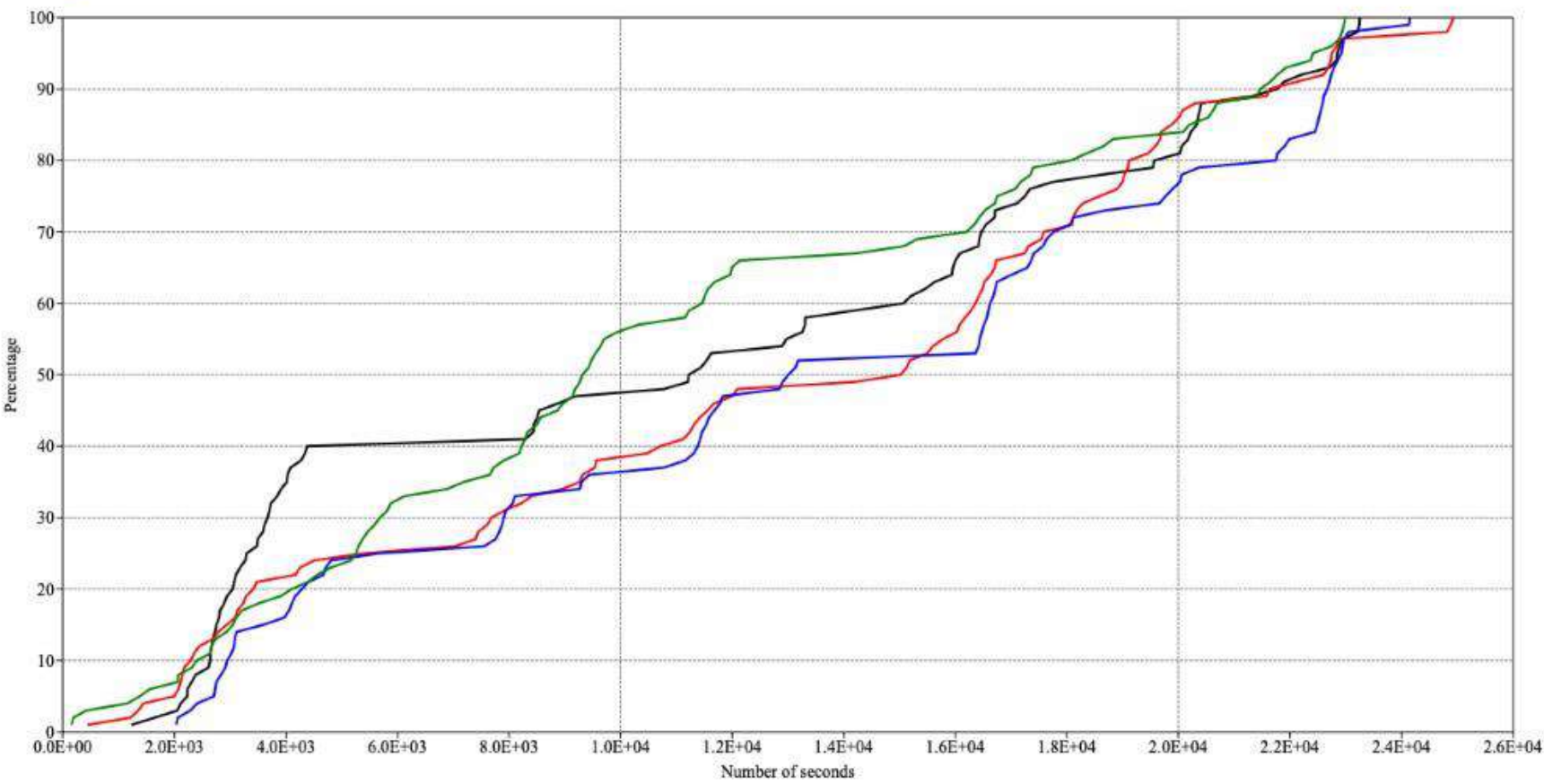


Ottosen et al, 2020. Genet. Biodiv. J, Special issue (Behavioural Instability), 1-60

- Jutta $18-10$ (Four)
- Sebastian $18-10$ (Four)

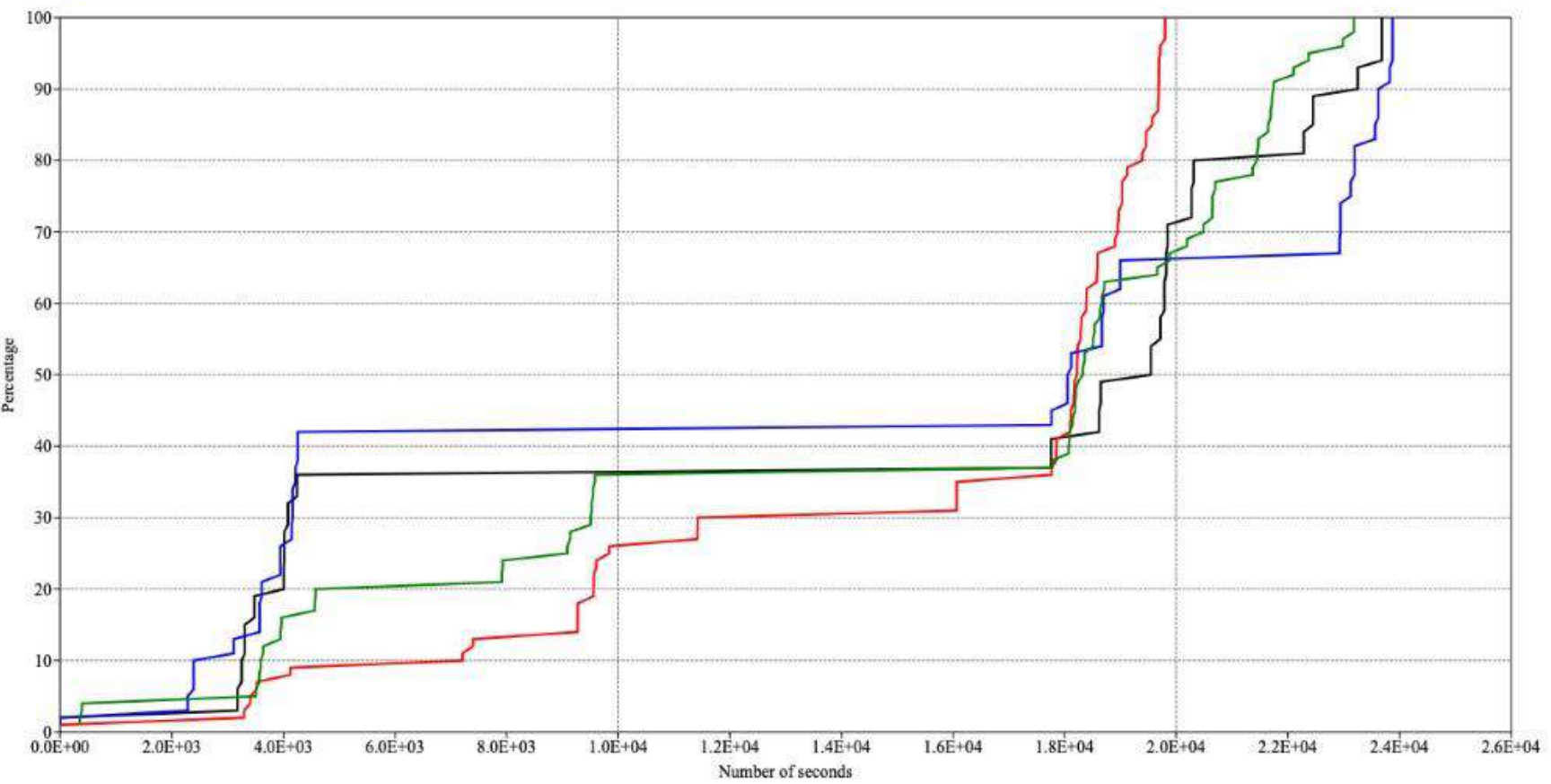

= Jutta $18-10$ (inter.)
= Sebastian 18 -10 (Inter.)
Laura $18-10$ (Inter.)

- My 18-10 (Inter.

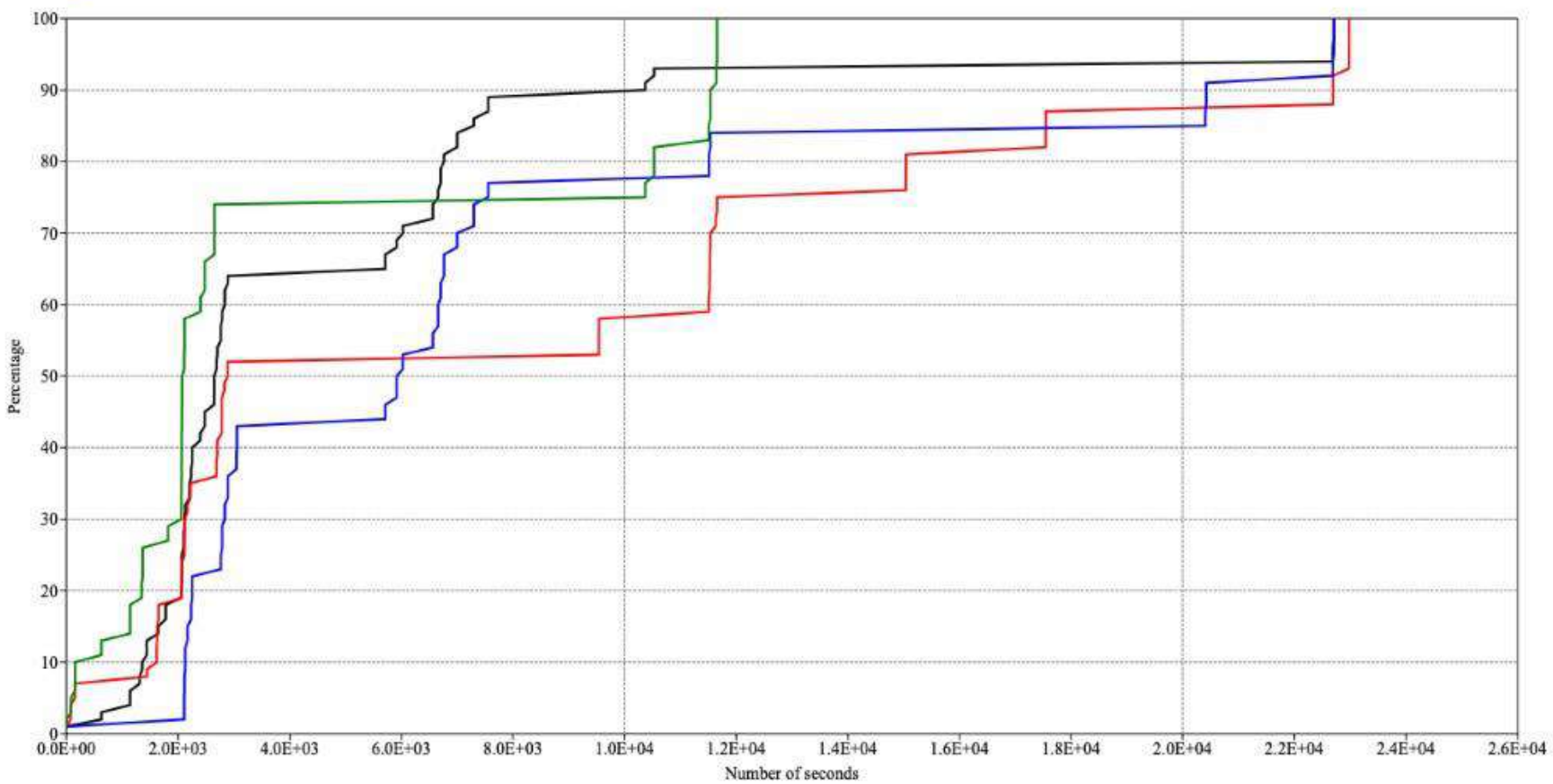


Ottosen et al, 2020. Genet. Biodiv. J, Special issue (Behavioural Instability), 1-60

20.10

- Jutta 20-10 (pass)

Sebastian $20-10$ (pas)
- Laura 20-10 (pas)

- My 20-10 (pas)

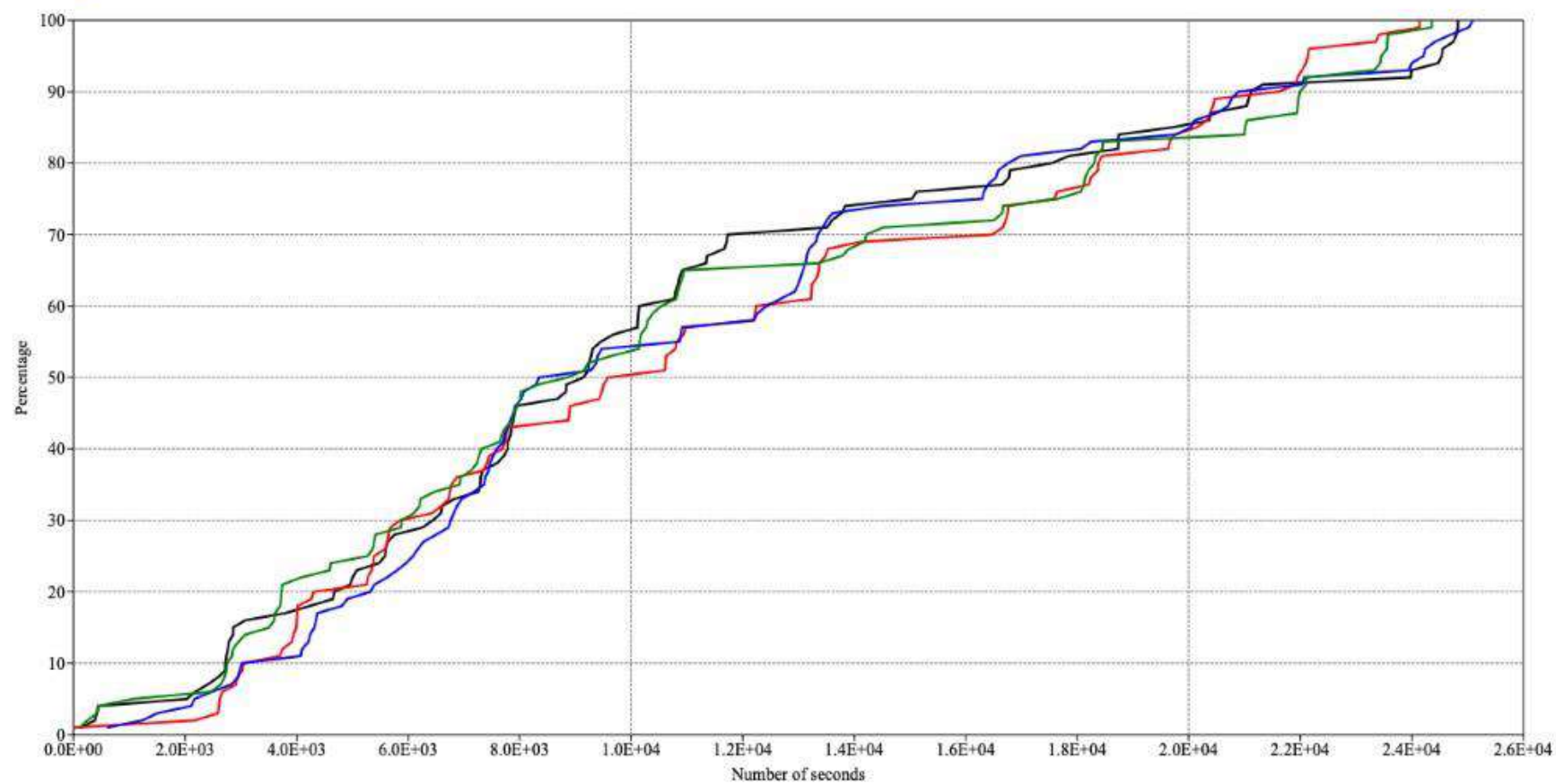


Ottosen et al, 2020. Genet. Biodiv. J, Special issue (Behavioural Instability), 1-60
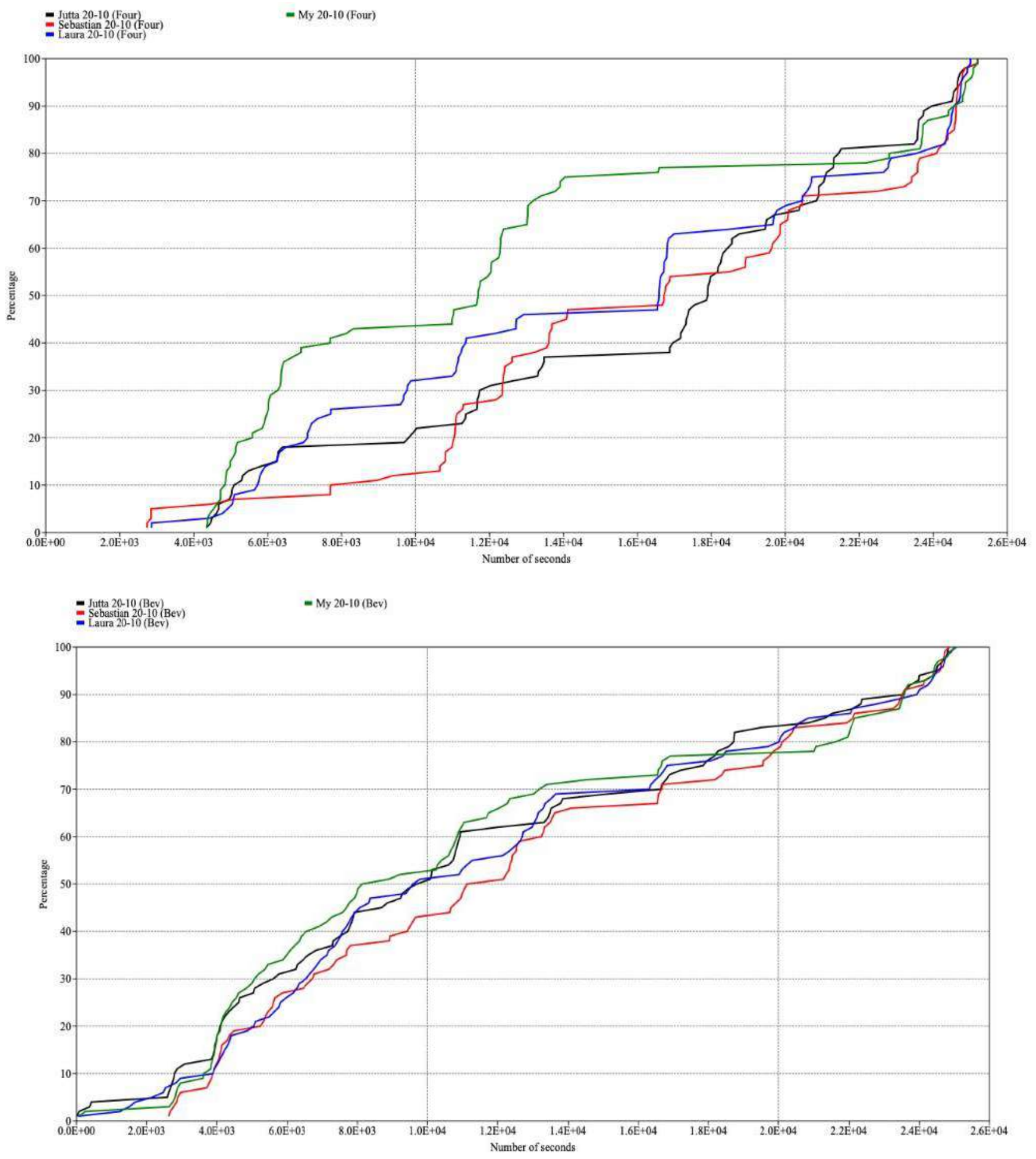
Ottosen et al, 2020. Genet. Biodiv. J, Special issue (Behavioural Instability), 1-60

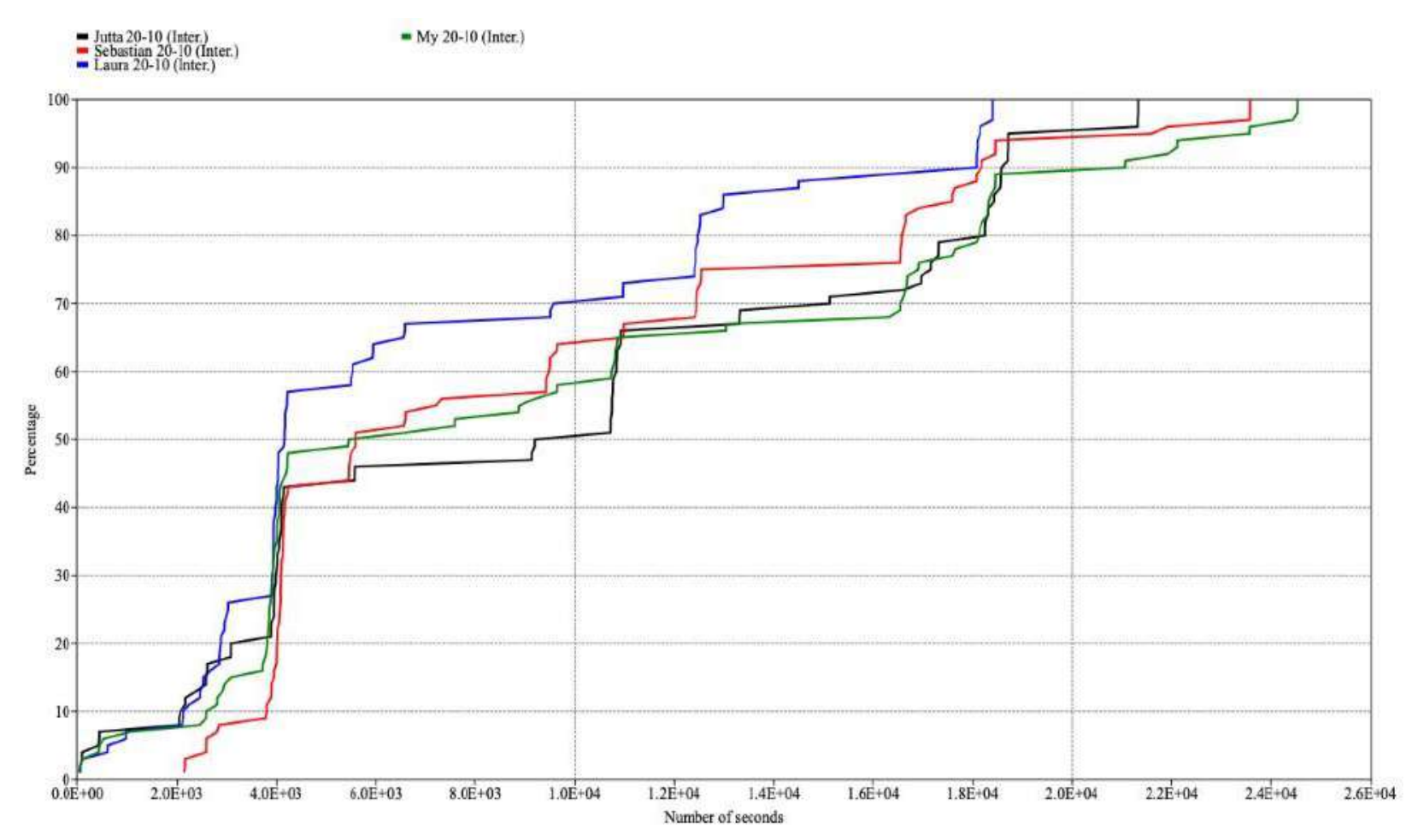


Ottosen et al, 2020. Genet. Biodiv. J, Special issue (Behavioural Instability), 1-60

\section{Appendix D}

\section{Time consumption}

\begin{tabular}{|c|c|c|c|c|c|c|c|}
\hline \multirow{2}{*}{ Control } & & \multicolumn{2}{|c|}{ Sebastian } & \multicolumn{2}{|l|}{ Laura } & \multicolumn{2}{|l|}{ Мy } \\
\hline & & Chi2 & $\mathrm{P}$ & Chi2 & $\mathrm{P}$ & Chi2 & $\mathrm{P}$ \\
\hline \multirow[t]{4}{*}{ Passive behaviour } & Jutta & 0.38 & 0.83 & 2.15 & 0.34 & 0.37 & 0.83 \\
\hline & Sebastian & & & 4.41 & 0.11 & 1.51 & 0.47 \\
\hline & Laura & & & & & 1.04 & 0.60 \\
\hline & My & & & & & & \\
\hline \multirow[t]{4}{*}{ Movement } & Jutta & 0.41 & 0.98 & 1.20 & 0.55 & 0.87 & 0.65 \\
\hline & Sebastian & & & 0.91 & 0.64 & 0.59 & 0.74 \\
\hline & Laura & & & & & 0.17 & 0.92 \\
\hline & My & & & & & & \\
\hline \multirow[t]{4}{*}{ Foraging } & Jutta & 0.65 & 0.72 & 1.25 & 0.54 & 1.43 & 0.49 \\
\hline & Sebastian & & & 0.58 & 0.75 & 0.49 & 0.78 \\
\hline & Laura & & & & & 1.90 & 0.39 \\
\hline & My & & & & & & \\
\hline \multirow[t]{4}{*}{ Reaction to guests/zoo keepers } & Jutta & 0 & 1 & 0 & 1 & 0 & 1 \\
\hline & Sebastian & & & - & - & - & - \\
\hline & Laura & & & & & - & - \\
\hline & My & & & & & & \\
\hline \multirow[t]{4}{*}{ Stereotype behaviour } & Jutta & 0 & 1 & 1.64 & 0.44 & 0.83 & 0.66 \\
\hline & Sebastian & & & 0 & 1 & 0 & 1 \\
\hline & Laura & & & & & 3.43 & 0.18 \\
\hline & My & & & & & & \\
\hline \multirow[t]{4}{*}{ Interaction } & Jutta & 8.58 & $0.014 *$ & 18.13 & $\begin{array}{l}0.00012 \\
* *\end{array}$ & 3.303 & $\begin{array}{l}6.72 * 10 \\
\wedge-8 * *\end{array}$ \\
\hline & Sebastian & & & 0.92 & 0.63 & 5.92 & 0.052 \\
\hline & Laura & & & & & 3.29 & 0.19 \\
\hline & My & & & & & & \\
\hline \multicolumn{8}{|l|}{ Holiday } \\
\hline \multirow[t]{4}{*}{ Passive behaviour } & Jutta & 0.65 & 0.72 & 0.42 & 0.81 & 1.20 & 0.55 \\
\hline & Sebastian & & & 1.98 & 0.37 & 3.66 & 0.16 \\
\hline & Laura & & & & & 0.62 & 0.73 \\
\hline & My & & & & & & \\
\hline \multirow[t]{4}{*}{ Movement } & Jutta & 0.24 & 0.89 & 0.12 & 0.94 & 0.57 & 0.75 \\
\hline & Sebastian & & & 0.62 & 0.74 & 0.48 & 0.79 \\
\hline & Laura & & & & & 1.45 & 0.48 \\
\hline & My & & & & & & \\
\hline \multirow[t]{4}{*}{ Foraging } & Jutta & 0.052 & 0.97 & 0.28 & 0.87 & 0.33 & 0.85 \\
\hline & Sebastian & & & 0.11 & 0.95 & 0.26 & 0.88 \\
\hline & Laura & & & & & 0.18 & 0.92 \\
\hline & My & & & & & & \\
\hline \multirow[t]{4}{*}{ Reaction to guests/zoo keepers } & Jutta & - & - & - & - & 0 & 1 \\
\hline & Sebastian & & & - & - & 0 & 1 \\
\hline & Laura & & & & & 0 & 1 \\
\hline & My & & & & & & \\
\hline \multirow[t]{4}{*}{ Stereotype behaviour } & Jutta & 0.92 & 0.63 & 0.86 & 0.65 & 0 & 1 \\
\hline & Sebastian & & & 1.2 & 0.27 & 0 & 1 \\
\hline & Laura & & & & & 0 & 1 \\
\hline & My & & & & & & \\
\hline \multirow[t]{4}{*}{ Interaction } & Jutta & 6.82 & $0.033^{*}$ & 3.58 & 0.17 & 8.39 & $0.015^{*}$ \\
\hline & Sebastian & & & 1.29 & 0.53 & 0.23 & 0.89 \\
\hline & Laura & & & & & 0.91 & 0.63 \\
\hline & My & & & & & & \\
\hline
\end{tabular}

$* \mathrm{P}<0.05$

$* * \mathrm{P}<0.01$

$¥$ Pga. nulrække var denne sammenligning ikke mulig 


\section{Appendix E}

\section{Kruskal Wallis and Mann-Whitney pairwise tests}

\section{Comparisons Between The Periods}

\section{Jutta}

Passive behaviour

Kruskal-Wallis test for equal medians

$\mathrm{H}$ (chi2):

Hc (tie corrected):

There is no significant difference between sample medians

\section{Movement}

Kruskal-Wallis test for equal medians

H (chi2):

Hc (tie corrected):

p (same):

There is a significant difference between sample medians

Foraging

Kruskal-Wallis test for equal medians

H (chi2):

$\mathrm{Hc}$ (tie corrected):

p (same):

There is a significant difference between sample medians

Interaction

Kruskal-Wallis test for equal medians H (chi2): Hc (tie corrected):

$\mathrm{p}$ (same):

There is a significant difference between sample medians

\section{Sebastian}

Passive behaviour

Kruskal-Wallis test for equal medians

H (chi2):

Hc (tie corrected):

p (same):

There is no significant difference between sample medians

Movement

Kruskal-Wallis test for equal medians

$\mathrm{H}$ (chi2):

$\mathrm{Hc}$ (tie corrected):

$\mathrm{p}$ (same):

There is no significant difference between sample medians 
Ottosen et al, 2020. Genet. Biodiv. J, Special issue (Behavioural Instability), 1-60

Foraging

Kruskal-Wallis test for equal medians

H (chi2):

Hc (tie corrected):

p (same):

There is no significant difference between sample medians

Interaction

Kruskal-Wallis test for equal medians

H (chi2):

Hc (tie corrected):

p (same):

There is no significant difference between sample medians

\section{Laura}

Passive behaviour

Kruskal-Wallis test for equal medians

$\mathrm{H}$ (chi2):

17.02

Hc (tie corrected):

17.02

p (same):

0.004454

There is a significant difference between sample medians

Movement

Kruskal-Wallis test for equal medians

$\mathrm{H}$ (chi2):

Hc (tie corrected):

$\mathrm{p}$ (same):

0.7899

There is no significant difference between sample medians

Foraging

Kruskal-Wallis test for equal medians

H (chi2):

Hc (tie corrected):

7.184

p (same):

0.2073

There is no significant difference between sample medians

Stereotype behaviour

Kruskal-Wallis test for equal medians

$\mathrm{H}$ (chi2):

0.1026

Hc (tie corrected):

0.1026

p (same):

0.7488 
Ottosen et al, 2020. Genet. Biodiv. J, Special issue (Behavioural Instability), 1-60

There is no significant difference between sample medians

Interaction

Kruskal-Wallis test for equal medians

H (chi2):

Hc (tie corrected):

p (same):

There is no significant difference between sample medians

\section{My}

Passive behaviour

Kruskal-Wallis test for equal medians

H (chi2):

$\mathrm{Hc}$ (tie corrected):

p (same):

There is a significant difference between sample medians

Movement

Kruskal-Wallis test for equal medians

H (chi2):

Hc (tie corrected):

18.12

$\mathrm{p}$ (same):

0.0028

There is a significant difference between sample medians

Foraging

Kruskal-Wallis test for equal medians

H (chi2):

Hc (tie corrected):

10.28

$\mathrm{p}$ (same):

0.06766

There is no significant difference between sample medians Reaction to guests/zookeepers

Kruskal-Wallis test for equal medians

$\mathrm{H}$ (chi2):

Hc (tie corrected):

8.112

$\mathrm{p}$ (same):

0.01732

There is a significant difference between sample medians Interaction 
Ottosen et al, 2020. Genet. Biodiv. J, Special issue (Behavioural Instability), 1-60

Kruskal-Wallis test for equal medians

H (chi2):

Hc (tie corrected):

p (same):

0.02609

There is a significant difference between sample medians

\section{Mann-Whitnet Pairwise}

\begin{tabular}{|c|c|c|c|c|c|c|c|c|}
\hline & & & $\mathrm{C} 1$ & $\mathrm{C} 2$ & $\mathrm{C} 3$ & $\mathrm{H} 1$ & $\mathrm{H} 2$ & $\mathrm{H} 3$ \\
\hline \multirow{24}{*}{$\begin{array}{l}\text { Passive } \\
\text { behaviour }\end{array}$} & \multirow[t]{6}{*}{ Jutta } & $\mathrm{C} 1$ & & $\mathrm{C} 2>\mathrm{C} 1 *$ & n.s. & n.s. & n.s. & n.s. \\
\hline & & $\mathrm{C} 2$ & $\mathrm{C} 2>\mathrm{C} 1 *$ & & $\mathrm{C} 2>\mathrm{C} 3 *$ & n.s. & n.s. & $\mathrm{C} 2>\mathrm{H} 3 *$ \\
\hline & & C3 & n.s. & $\mathrm{C} 2>\mathrm{C} 3 *$ & & n.s. & n.s. & n.s. \\
\hline & & $\mathrm{H} 1$ & n.s. & n.s. & n.s. & & n.s. & n.s. \\
\hline & & $\mathrm{H} 2$ & n.s. & n.s. & n.s. & n.s. & & n.s. \\
\hline & & $\mathrm{H} 3$ & n.s. & $\mathrm{C} 2>\mathrm{H} 3 *$ & n.s. & n.s. & n.s. & \\
\hline & \multirow[t]{6}{*}{ Sebastian } & $\mathrm{C} 1$ & & n.s. & $\mathrm{C} 1>\mathrm{C} 3 *$ & n.s. & n.s. & n.s. \\
\hline & & $\mathrm{C} 2$ & n.s. & & n.s. & n.s. & n.s. & n.s. \\
\hline & & $\mathrm{C} 3$ & $\mathrm{C} 1>\mathrm{C} 3 *$ & n.s. & & n.s. & n.s. & n.s. \\
\hline & & $\mathrm{H} 1$ & n.s. & n.s. & n.s. & & n.s. & n.s. \\
\hline & & $\mathrm{H} 2$ & n.s. & n.s. & n.s. & n.s. & & n.s. \\
\hline & & $\mathrm{H} 3$ & n.s. & n.s. & n.s. & n.s. & n.s. & \\
\hline & \multirow[t]{6}{*}{ Laura } & $\mathrm{C} 1$ & & $\mathrm{C} 2>\mathrm{C} 1 * *$ & n.s. & n.s. & $\mathrm{H} 2>\mathrm{C} 1 *$ & n.s. \\
\hline & & $\mathrm{C} 2$ & $\mathrm{C} 2>\mathrm{C} 1 * *$ & & $\mathrm{C} 2>\mathrm{C} 3 *$ & n.s. & n.s. & $\mathrm{C} 2>\mathrm{H} 3 * *$ \\
\hline & & $\mathrm{C} 3$ & n.s. & $\mathrm{C} 2>\mathrm{C} 3 *$ & & n.s. & n.s. & n.s. \\
\hline & & $\mathrm{H} 1$ & n.s. & n.s. & n.s. & & n.s. & $\mathrm{H} 1>\mathrm{H} 3 *$ \\
\hline & & $\mathrm{H} 2$ & $\mathrm{H} 2>\mathrm{C} 1 *$ & n.s. & n.s. & n.s. & & $\mathrm{H} 2>\mathrm{H} 3 * *$ \\
\hline & & $\mathrm{H} 3$ & n.s. & $\mathrm{C} 2>\mathrm{H} 3 * *$ & n.s. & $\mathrm{H} 1>\mathrm{H} 3 *$ & $\mathrm{H} 2>\mathrm{H} 3 * *$ & \\
\hline & \multirow[t]{6}{*}{ My } & $\mathrm{C} 1$ & & n.s. & $\mathrm{C} 1>\mathrm{C} 3 *$ & $\mathrm{C} 1>\mathrm{H} 1 * *$ & n.s. & $\mathrm{C} 1>\mathrm{H} 3 *$ \\
\hline & & $\mathrm{C} 2$ & n.s. & & n.s. & $\mathrm{C} 2>\mathrm{H} 1 *$ & n.s. & n.s. \\
\hline & & $\mathrm{C} 3$ & $\mathrm{C} 1>\mathrm{C} 3 *$ & n.s. & & n.s. & $\mathrm{H} 2>\mathrm{C} 3 *$ & n.s. \\
\hline & & $\mathrm{H} 1$ & $\mathrm{C} 1>\mathrm{H} 1 * *$ & $\mathrm{C} 2>\mathrm{H} 1 *$ & n.s. & & $\mathrm{H} 2>\mathrm{H} 1 * *$ & n.s. \\
\hline & & $\mathrm{H} 2$ & n.s. & n.s. & $\mathrm{H} 2>\mathrm{C} 3 *$ & $\mathrm{H} 2>\mathrm{H} 1 * *$ & & $\mathrm{H} 2>\mathrm{H} 3 *$ \\
\hline & & $\mathrm{H} 3$ & $\mathrm{C} 1>\mathrm{H} 3 *$ & n.s. & n.s. & n.s. & $\mathrm{H} 2>\mathrm{H} 3{ }^{*}$ & \\
\hline \multirow[t]{15}{*}{ Movement } & \multirow[t]{6}{*}{ Jutta } & $\mathrm{C} 1$ & & n.s. & n.s. & $\mathrm{H} 1>\mathrm{C} 1 * *$ & n.s. & n.s. \\
\hline & & $\mathrm{C} 2$ & n.s. & & n.s. & $\mathrm{H} 1>\mathrm{C} 2 * *$ & n.s. & n.s. \\
\hline & & $\mathrm{C} 3$ & n.s. & n.s. & & $\mathrm{H} 1>\mathrm{C} 3 * *$ & n.s. & n.s. \\
\hline & & $\mathrm{H} 1$ & $\mathrm{H} 1>\mathrm{C} 1 * *$ & $\mathrm{H} 1>\mathrm{C} 2 * *$ & $\mathrm{H} 1>\mathrm{C} 3 * *$ & & $\mathrm{H} 1>\mathrm{H} 2 *$ & $\mathrm{H} 1>\mathrm{H} 3 *$ \\
\hline & & $\mathrm{H} 2$ & n.s. & n.s. & n.s. & $\mathrm{H} 1>\mathrm{H} 2 *$ & & n.s. \\
\hline & & $\mathrm{H} 3$ & n.s. & n.s. & n.s. & $\mathrm{H} 1>\mathrm{H} 3 *$ & n.s. & \\
\hline & \multirow[t]{6}{*}{ Sebastian } & $\mathrm{C} 1$ & & n.s. & n.s. & n.s. & $\mathrm{C} 1>\mathrm{H} 2 * *$ & n.s. \\
\hline & & $\mathrm{C} 2$ & n.s. & & n.s. & n.s. & $\mathrm{C} 2>\mathrm{H} 2 *$ & n.s. \\
\hline & & C3 & n.s. & n.s. & & n.s. & n.s. & n.s. \\
\hline & & $\mathrm{H} 1$ & n.s. & n.s. & n.s. & & n.s. & n.s. \\
\hline & & $\mathrm{H} 2$ & $\mathrm{C} 1>\mathrm{H} 2 * *$ & $\mathrm{C} 2>\mathrm{H} 2 *$ & n.s. & n.s. & & $\mathrm{H} 3>\mathrm{H} 2 *$ \\
\hline & & $\mathrm{H} 3$ & n.s. & n.s. & n.s. & n.s. & $\mathrm{H} 3>\mathrm{H} 2 *$ & \\
\hline & \multirow[t]{3}{*}{ Laura } & $\mathrm{C} 1$ & & n.s. & n.s. & n.s. & n.s. & n.s. \\
\hline & & $\mathrm{C} 2$ & n.s. & & n.s. & n.s. & n.s. & n.s. \\
\hline & & $\mathrm{C} 3$ & n.s. & n.s. & & n.s. & n.s. & n.s. \\
\hline & & $\mathrm{H} 1$ & n.s. & n.s. & n.s. & & n.s. & n.s. \\
\hline & & $\mathrm{H} 2$ & n.s. & n.s. & n.s. & n.s. & & n.s. \\
\hline & & $\mathrm{H} 3$ & n.s. & n.s. & n.s. & n.s. & n.s. & \\
\hline & My & C1 & & $\mathrm{C} 1>\mathrm{C} 2 *$ & $\mathrm{C} 1>\mathrm{C} 3 * *$ & $\mathrm{C} 1>\mathrm{H} 1 * *$ & $\mathrm{C} 1>\mathrm{H} 2 *$ & $\mathrm{C} 1>\mathrm{H} 3 * *$ \\
\hline & & $\mathrm{C} 2$ & $\mathrm{C} 1>\mathrm{C} 2 *$ & & n.s. & n.s. & n.s. & n.s. \\
\hline & & C3 & $\mathrm{C} 1>\mathrm{C} 3 * *$ & n.s. & & n.s. & n.s. & n.s. \\
\hline & & $\mathrm{H} 1$ & $\mathrm{C} 1>\mathrm{H} 1 * *$ & n.s. & n.s. & & $\mathrm{H} 2>\mathrm{H} 1 *$ & n.s. \\
\hline & & $\mathrm{H} 2$ & $\mathrm{C} 1>\mathrm{H} 2 *$ & n.s. & n.s. & $\mathrm{H} 2>\mathrm{H} 1 *$ & & n.s. \\
\hline & & $\mathrm{H} 3$ & $\mathrm{C} 1>\mathrm{H} 3 * *$ & n.s. & n.s. & n.s. & n.s. & \\
\hline Foraging & Jutta & $\mathrm{C} 1$ & & n.s. & n.s. & $\mathrm{H} 1>\mathrm{C} 1 * *$ & n.s. & n.s. \\
\hline
\end{tabular}


Ottosen et al, 2020. Genet. Biodiv. J, Special issue (Behavioural Instability), 1-60

\begin{tabular}{|c|c|c|c|c|c|c|c|c|}
\hline & & $\mathrm{C} 2$ & n.s. & & n.s. & n.s. & n.s. & n.s. \\
\hline & & C3 & n.s. & n.s. & & $\mathrm{H} 1>\mathrm{C} 3 * *$ & n.s. & n.s. \\
\hline & & H1 & $\mathrm{H} 1>\mathrm{C} 1 * *$ & n.s. & $\mathrm{H} 1>\mathrm{C} 3 * *$ & & n.s. & $\mathrm{H} 1>\mathrm{H} 3 *$ \\
\hline & & $\mathrm{H} 2$ & n.s. & n.s. & n.s. & n.s. & & n.s. \\
\hline & & $\mathrm{H} 3$ & n.s. & n.s. & n.s. & $\mathrm{H} 1>\mathrm{H} 3 *$ & n.s. & \\
\hline & \multirow[t]{6}{*}{ Sebastian } & $\mathrm{C} 1$ & & $\mathrm{C} 2>\mathrm{C} 1 *$ & n.s. & $\mathrm{H} 1>\mathrm{C} 1 *$ & n.s. & n.s. \\
\hline & & $\mathrm{C} 2$ & $\mathrm{C} 2>\mathrm{C} 1 *$ & & n.s. & n.s. & n.s. & n.s. \\
\hline & & C3 & n.s. & n.s. & & n.s. & n.s. & n.s. \\
\hline & & H1 & $\mathrm{H} 1>\mathrm{C} 1 *$ & n.s. & n.s. & & n.s. & n.s. \\
\hline & & $\mathrm{H} 2$ & n.s. & n.s. & n.s. & n.s. & & n.s. \\
\hline & & $\mathrm{H} 3$ & n.s. & n.s. & n.s. & n.s. & n.s. & \\
\hline & \multirow[t]{6}{*}{ Laura } & $\mathrm{C} 1$ & & n.s. & n.s. & n.s. & n.s. & n.s. \\
\hline & & $\mathrm{C} 2$ & n.s. & & n.s. & n.s. & n.s. & $\mathrm{C} 2>\mathrm{H} 3 *$ \\
\hline & & C3 & n.s. & n.s. & & n.s. & n.s. & n.s. \\
\hline & & $\mathrm{H} 1$ & n.s. & n.s. & n.s. & & n.s. & n.s. \\
\hline & & $\mathrm{H} 2$ & n.s. & n.s. & n.s. & n.s. & & n.s. \\
\hline & & H3 & n.s. & $\mathrm{C} 2>\mathrm{H} 3 *$ & n.s. & n.s. & n.s. & \\
\hline & \multirow[t]{6}{*}{ My } & $\mathrm{C} 1$ & & n.s. & n.s. & n.s. & n.s. & $\mathrm{H} 3>\mathrm{C} 1 *$ \\
\hline & & $\mathrm{C} 2$ & n.s. & & n.s. & n.s. & n.s. & n.s. \\
\hline & & C3 & n.s. & n.s. & & n.s. & n.s. & n.s. \\
\hline & & H1 & n.s. & n.s. & n.s. & & $\mathrm{H} 2>\mathrm{H} 1 *$ & $\mathrm{H} 3>\mathrm{H} 1 * *$ \\
\hline & & $\mathrm{H} 2$ & n.s. & n.s. & n.s. & $\mathrm{H} 2>\mathrm{H} 1 *$ & & n.s. \\
\hline & & H3 & $\mathrm{H} 3>\mathrm{C} 1 *$ & n.s. & n.s. & $\mathrm{H} 3>\mathrm{H} 1 * *$ & n.s. & \\
\hline \multirow{18}{*}{$\begin{array}{l}\text { Reaction to } \\
\text { guests/zook } \\
\text { eepers }\end{array}$} & \multirow[t]{6}{*}{ Jutta } & $\mathrm{C} 1$ & & - & - & - & - & - \\
\hline & & $\mathrm{C} 2$ & - & & - & - & - & - \\
\hline & & C3 & - & - & & - & - & - \\
\hline & & H1 & - & - & - & & - & - \\
\hline & & $\mathrm{H} 2$ & - & - & - & - & & - \\
\hline & & $\mathrm{H} 3$ & - & - & - & - & - & \\
\hline & \multirow[t]{6}{*}{ Sebastian } & $\mathrm{C} 1$ & & - & - & - & - & - \\
\hline & & $\mathrm{C} 2$ & - & 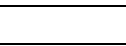 & - & - & - & - \\
\hline & & C3 & - & - & & - & - & - \\
\hline & & H1 & - & - & - & & - & - \\
\hline & & $\mathrm{H} 2$ & - & - & - & - & & - \\
\hline & & H3 & - & - & - & - & - & \\
\hline & \multirow[t]{6}{*}{ Laura } & $\mathrm{C} 1$ & & - & - & - & - & - \\
\hline & & $\mathrm{C} 2$ & - & & - & - & - & - \\
\hline & & C3 & - & - & & - & - & - \\
\hline & & H1 & - & - & - & & - & - \\
\hline & & $\mathrm{H} 2$ & - & - & - & - & & - \\
\hline & & H3 & - & - & - & - & - & \\
\hline
\end{tabular}


Ottosen et al, 2020. Genet. Biodiv. $J$, Special issue (Behavioural Instability), 1-60

\begin{tabular}{|c|c|c|c|c|c|c|c|c|}
\hline & \multirow[t]{6}{*}{ My } & $\mathrm{C} 1$ & & - & - & - & - & - \\
\hline & & $\mathrm{C} 2$ & - & & n.s. & $\mathrm{C} 2>\mathrm{H} 1 * *$ & - & - \\
\hline & & C3 & - & n.s. & & n.s. & - & - \\
\hline & & H1 & - & $\mathrm{C} 2>\mathrm{H} 1 * *$ & n.s. & & - & - \\
\hline & & $\mathrm{H} 2$ & - & - & - & - & & - \\
\hline & & $\mathrm{H} 3$ & - & - & - & - & - & \\
\hline Stereotype & \multirow[t]{6}{*}{ Jutta } & $\mathrm{C} 1$ & & - & - & - & - & - \\
\hline \multirow[t]{23}{*}{ behaviour } & & $\mathrm{C} 2$ & - & & - & - & - & - \\
\hline & & $\mathrm{C} 3$ & - & - & & - & - & - \\
\hline & & H1 & - & - & - & 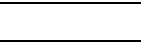 & - & - \\
\hline & & $\mathrm{H} 2$ & - & - & - & - & & - \\
\hline & & $\mathrm{H} 3$ & - & - & - & - & - & 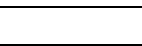 \\
\hline & \multirow[t]{6}{*}{ Sebastian } & $\mathrm{C} 1$ & & - & - & - & - & - \\
\hline & & $\mathrm{C} 2$ & - & & - & - & - & - \\
\hline & & $\mathrm{C} 3$ & - & - & & - & - & - \\
\hline & & H1 & - & - & - & 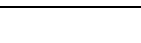 & - & - \\
\hline & & $\mathrm{H} 2$ & - & - & - & - & & - \\
\hline & & $\mathrm{H} 3$ & - & - & - & - & - & 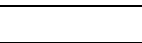 \\
\hline & \multirow[t]{6}{*}{ Laura } & $\mathrm{C} 1$ & & - & - & - & - & - \\
\hline & & $\mathrm{C} 2$ & - & 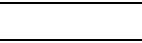 & - & - & - & - \\
\hline & & C3 & - & - & & - & - & n.s. \\
\hline & & H1 & - & - & - & & - & - \\
\hline & & $\mathrm{H} 2$ & - & - & - & - & 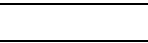 & - \\
\hline & & $\mathrm{H} 3$ & - & - & n.s. & - & - & \\
\hline & \multirow[t]{6}{*}{ My } & $\mathrm{C} 1$ & & - & - & - & - & - \\
\hline & & $\mathrm{C} 2$ & - & & - & - & - & - \\
\hline & & $\mathrm{C} 3$ & - & - & & - & - & - \\
\hline & & H1 & - & - & - & & - & - \\
\hline & & $\mathrm{H} 2$ & - & - & - & - & & - \\
\hline & & $\mathrm{H} 3$ & - & - & - & - & - & \\
\hline \multirow{24}{*}{ Interaction } & \multirow{6}{*}{ Jutta } & $\mathrm{C} 1$ & & n.s. & n.s. & n.s. & $\mathrm{H} 2>\mathrm{C} 1 *$ & n.s. \\
\hline & & $\mathrm{C} 2$ & n.s. & & n.s. & n.s. & $\mathrm{H} 2>\mathrm{C} 2 *$ & n.s. \\
\hline & & $\mathrm{C} 3$ & n.s. & n.s. & & n.s. & $\mathrm{H} 2>\mathrm{C} 3 *$ & n.s. \\
\hline & & H1 & n.s. & n.s. & n.s. & & $\mathrm{H} 2>\mathrm{H} 1 * *$ & n.s. \\
\hline & & $\mathrm{H} 2$ & $\mathrm{H} 2>\mathrm{C} 1 *$ & $\mathrm{H} 2>\mathrm{C} 2 *$ & $\mathrm{H} 2>\mathrm{C} 3 *$ & $\mathrm{H} 2>\mathrm{H} 1 * * *$ & & $\mathrm{H} 2>\mathrm{H} 3 * *$ \\
\hline & & $\mathrm{H} 3$ & n.s. & n.s. & n.s. & n.s. & $\mathrm{H} 2>\mathrm{H} 3 * *$ & \\
\hline & \multirow[t]{6}{*}{ Sebastian } & $\mathrm{C} 1$ & & n.s. & n.s. & n.s. & n.s. & C1>H3* \\
\hline & & $\mathrm{C} 2$ & n.s. & & n.s. & n.s. & n.s. & n.s. \\
\hline & & $\mathrm{C} 3$ & n.s. & n.s. & & n.s. & n.s. & n.s. \\
\hline & & H1 & n.s. & n.s. & n.s. & & n.s. & n.s. \\
\hline & & $\mathrm{H} 2$ & $\begin{array}{ll}\text { n.s. } \\
\text { n. }\end{array}$ & n.s. & n.s. & n.s. & & n.s. \\
\hline & & $\mathrm{H} 3$ & $\mathrm{C} 1>\mathrm{H} 3 *$ & n.s. & n.s. & n.s. & n.s. & \\
\hline & \multirow[t]{6}{*}{ Laura } & $\mathrm{C} 1$ & & n.s. & n.s. & n.s. & n.s. & n.s. \\
\hline & & $\mathrm{C} 2$ & n.s. & & n.s. & n.s. & n.s. & n.s. \\
\hline & & C3 & n.s. & n.s. & & n.s. & n.s. & n.s. \\
\hline & & H1 & n.s. & n.s. & n.s. & & n.s. & n.s. \\
\hline & & $\mathrm{H} 2$ & n.s. & n.s. & n.s. & n.s. & & n.s. \\
\hline & & $\mathrm{H} 3$ & n.s. & n.s. & n.s. & n.s. & n.s. & \\
\hline & \multirow[t]{3}{*}{ My } & $\mathrm{C} 1$ & & n.s. & $\mathrm{C} 1>\mathrm{C} 3^{*}$ & $\mathrm{C} 1>\mathrm{H} 1 * *$ & n.s. & $\mathrm{C} 1>\mathrm{H} 3 * *$ \\
\hline & & $\mathrm{C} 2$ & n.s. & & n.s. & n.s. & n.s. & n.s. \\
\hline & & $\mathrm{C} 3$ & $\mathrm{C} 1>\mathrm{C} 3^{*}$ & n.s. & & n.s. & n.s. & n.s. \\
\hline & & H1 & $\mathrm{C} 1>\mathrm{H} 1 * *$ & n.s. & n.s. & & n.s. & n.s. \\
\hline & & $\mathrm{H} 2$ & n.s. & n.s. & n.s. & n.s. & & n.s. \\
\hline & & $\mathrm{H} 3$ & $\mathrm{C} 1>\mathrm{H} 3 * *$ & n.s. & n.s. & n.s. & n.s. & \\
\hline$* \mathrm{P}<0.05$ & & & & & & & & \\
\hline$* * \mathrm{P}<0.01$ & & & & & & & & \\
\hline
\end{tabular}

\section{Appendix F}


Ottosen et al, 2020. Genet. Biodiv. J, Special issue (Behavioural Instability), 1-60

\section{Summary Statistics For Comparisons Between The Periods \\ Jutta}

Passive

\begin{tabular}{|c|c|c|c|c|c|c|c|c|c|c|c|c|c|}
\hline \multirow[t]{2}{*}{ Control } & \multirow[b]{2}{*}{$30-09$} & \multirow[b]{2}{*}{$\begin{array}{l}\text { Lower } \\
\text { conf. }\end{array}$} & \multirow{2}{*}{\multicolumn{2}{|c|}{$\begin{array}{l}\text { Upper } \\
\text { conf. } \\
\end{array}$}} & \multirow{2}{*}{\multicolumn{2}{|c|}{$02-10$}} & \multirow{2}{*}{\multicolumn{2}{|c|}{$\begin{array}{l}\text { Lower } \\
\text { conf. }\end{array}$}} & \multirow{2}{*}{\multicolumn{2}{|c|}{$\begin{array}{l}\text { Upper } \\
\text { conf. }\end{array}$}} & \multirow[b]{2}{*}{$05-10$} & \multirow[b]{2}{*}{$\begin{array}{l}\text { Lower } \\
\text { conf. }\end{array}$} & \multirow[b]{2}{*}{$\begin{array}{l}\text { Upper } \\
\text { conf. }\end{array}$} \\
\hline & & & & & & & & & & & & & \\
\hline $\mathrm{N}$ & 53 & 53 & \multicolumn{2}{|c|}{53} & \multicolumn{2}{|c|}{39} & \multicolumn{2}{|c|}{39} & \multicolumn{2}{|l|}{39} & 69 & 69 & 69 \\
\hline Min & 3 & & & & \multicolumn{2}{|c|}{10} & & \multicolumn{2}{|l|}{59} & 10 & & \\
\hline Max & 2141 & & \multicolumn{2}{|c|}{ 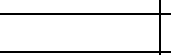 } & \multicolumn{2}{|c|}{3651} & & & & & 1338 & & \\
\hline Sum & 8651 & 2637 & 132 & & 1178 & & 288 & & 18238 & & 7926 & 4168 & 10916 \\
\hline Mean & 163.23 & 49.75 & 250 & & 302.2 & & 73.9 & & 467.64 & & 114.87 & 60.41 & 158.20 \\
\hline Std. error & 51.93 & 0.00 & 71. & & 103.8 & & 0.00 & & 142.07 & & 25.54 & 0.00 & 33.66 \\
\hline Variance & $\begin{array}{l}142907.5 \\
0\end{array}$ & $\begin{array}{l}- \\
31806.39\end{array}$ & $\begin{array}{l}270 \\
0\end{array}$ & 542.2 & $\begin{array}{l}4207 \\
0\end{array}$ & 43.4 & $\begin{array}{l}- \\
149 \\
0\end{array}$ & 8.6 & $\begin{array}{l}787158 \\
0\end{array}$ & & $\begin{array}{l}45017.7 \\
0\end{array}$ & $\begin{array}{l}- \\
4075.0 \\
6\end{array}$ & $\begin{array}{l}78191.9 \\
6\end{array}$ \\
\hline Stand. dev & 378.03 & 192.48 & 632 & & 648. & & 301 & & 1064.21 & & 212.17 & 117.57 & 315.52 \\
\hline Median & 28.00 & 6.00 & 32. & & 66.00 & & 27.0 & & 88.00 & & 31.00 & 12.00 & 34.00 \\
\hline 25 prentil & 18.50 & 13.00 & 22. & & 27.00 & & 5.00 & & 34.00 & & 17.00 & 9.00 & 19.50 \\
\hline 75 prentil & 104.00 & -33.00 & 158 & & 255. & & $-18 c$ & & 419.00 & & 93.00 & -7.50 & 127.00 \\
\hline Skewness & 3.97 & 2.10 & 5.7 & & 4.03 & & 2.95 & & 6.60 & & 3.69 & 2.40 & 5.52 \\
\hline Kurtosis & 17.05 & -3.74 & 29. & & $19.0^{\prime}$ & & 9.88 & & 37.48 & & 16.75 & 3.30 & 31.03 \\
\hline $\begin{array}{l}\text { Geom. } \\
\text { mean }\end{array}$ & 47.12 & 24.27 & 61. & & 89.8 & & 37.8 & & 121.68 & & 46.71 & 30.34 & 57.84 \\
\hline Coeff. var & 231.60 & 176.73 & 317 & & 214. & & 154 & & 294.12 & & 184.71 & 143.90 & 235.08 \\
\hline Holiday & & & & & & & & & & & & & \\
\hline $16-10$ & $\begin{array}{l}\text { Lower } \\
\text { conf. }\end{array}$ & $\begin{array}{l}\text { Upper } \\
\text { conf. }\end{array}$ & & $18-10$ & & $\begin{array}{l}\text { Low } \\
\text { conf. }\end{array}$ & & $\begin{array}{l}\mathrm{Up} \\
\mathrm{co}\end{array}$ & & 20 & & $\begin{array}{l}\text { Lower } \\
\text { conf. }\end{array}$ & $\begin{array}{l}\text { Upper } \\
\text { conf. }\end{array}$ \\
\hline 19 & 19 & 19 & & 68 & & 68 & & 68 & & 64 & & 64 & 64 \\
\hline 10 & & & & 7 & & & & & & 3 & & & \\
\hline 4342 & & & & 1316 & & & & & & 15 & & & \\
\hline 9262 & -1387 & 17185 & & 13319 & & 7768 & & & & 87 & & 4435 & 12246 \\
\hline 487.47 & -73.00 & 904.47 & & 195.87 & & 114.2 & & & & & .33 & 69.30 & 191.34 \\
\hline 258.58 & 0.00 & 364.91 & & 40.16 & & 25.31 & & 50 & & 31 & & 0.00 & 42.12 \\
\hline $\begin{array}{l}1270382.0 \\
0\end{array}$ & $\begin{array}{l}- \\
204538.6 \\
0\end{array}$ & $\begin{array}{l}2529966 \\
0\end{array}$ & & 109672.5 & & 4352 & 5.82 & & 19.40 & & 12.83 & -9406.74 & 113549.80 \\
\hline 1127.11 & 597.33 & 2150.31 & & 331.17 & & 243. & & & & & 3.01 & 135.30 & 385.70 \\
\hline 38.00 & 6.00 & 54.00 & & 62.00 & & 24.50 & & 86 & & 35 & & -1.00 & 51.00 \\
\hline 18.00 & 2.00 & 25.00 & & 24.25 & & 14.5 & & 33 & & 15 & & 12.00 & 18.25 \\
\hline 150.00 & -2231.00 & 250.00 & & 158.50 & & 13.5 & & & & & .25 & 82.00 & 221.00 \\
\hline 2.83 & 1.32 & 4.40 & & 2.44 & & 1.15 & & 3.2 & & 3.8 & & 2.46 & 5.78 \\
\hline 7.94 & -2.95 & 15.87 & & 4.99 & & -5.31 & & 8.7 & & 17 & & 3.92 & 33.22 \\
\hline 71.68 & -24.70 & 108.83 & & 70.52 & & 43.1 & & 90 & & 48 & & 28.22 & 62.13 \\
\hline 231.22 & 116.02 & 331.89 & & 169.08 & & 142. & & & & & .59 & 142.67 & 242.50 \\
\hline
\end{tabular}


Ottosen et al, 2020. Genet. Biodiv. J, Special issue (Behavioural Instability), 1-60

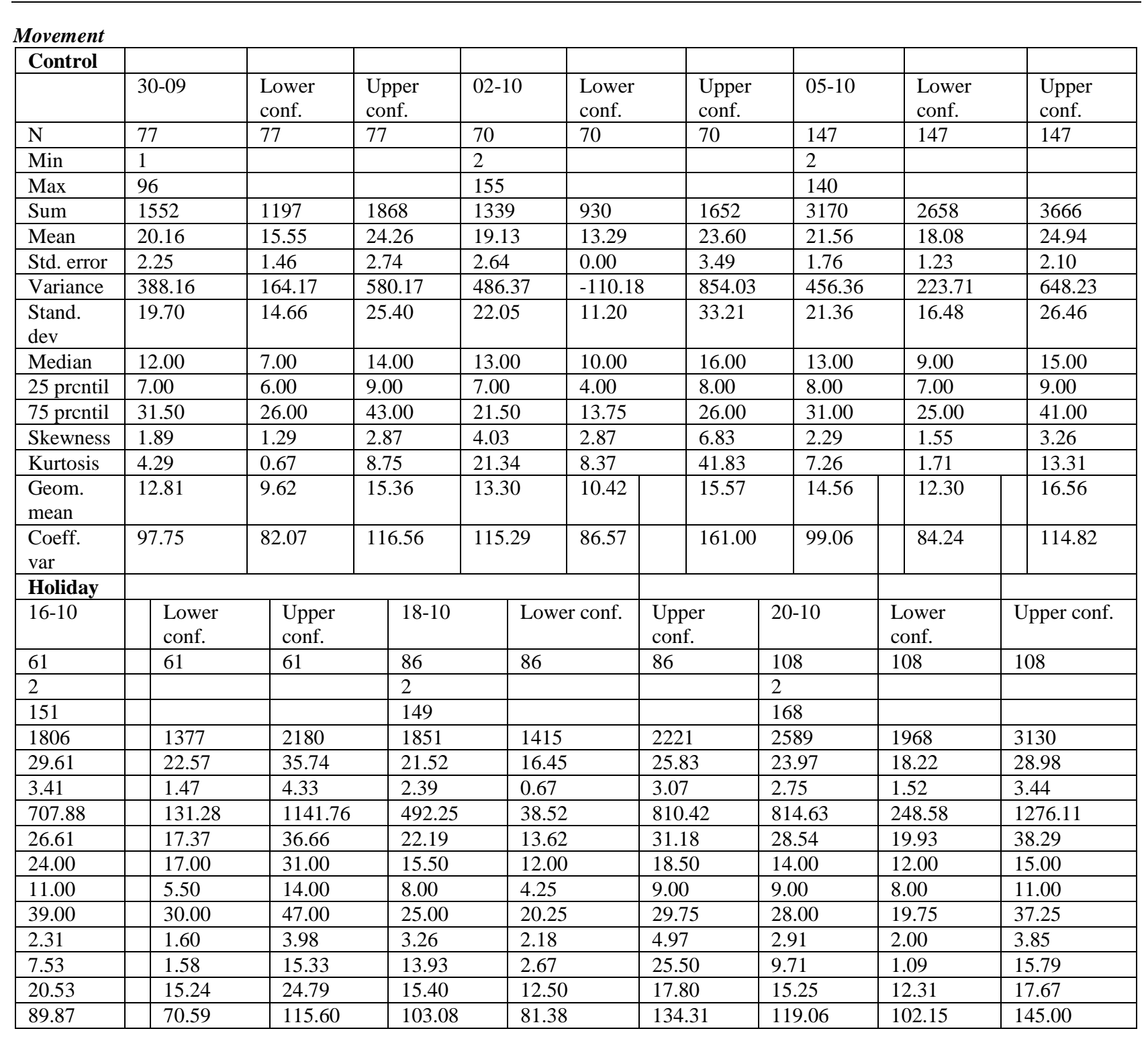


Ottosen et al, 2020. Genet. Biodiv. J, Special issue (Behavioural Instability), 1-60

\begin{tabular}{|c|c|c|c|c|c|c|c|c|c|c|c|}
\hline \multicolumn{12}{|c|}{ Foraging } \\
\hline \multirow[t]{2}{*}{ Control } & \multirow[b]{2}{*}{$30-09$} & \multirow[b]{2}{*}{$\begin{array}{l}\text { Lower } \\
\text { conf. }\end{array}$} & \multirow[b]{2}{*}{$\begin{array}{l}\text { Upper } \\
\text { conf. }\end{array}$} & \multirow{2}{*}{\multicolumn{2}{|c|}{$02-10$}} & \multirow{2}{*}{\multicolumn{2}{|c|}{$\begin{array}{l}\text { Lower } \\
\text { conf. }\end{array}$}} & \multirow[b]{2}{*}{$\begin{array}{l}\text { Upper } \\
\text { conf. }\end{array}$} & \multirow[b]{2}{*}{$05-10$} & \multirow[b]{2}{*}{$\begin{array}{l}\text { Lower } \\
\text { conf. }\end{array}$} & \multirow[b]{2}{*}{$\begin{array}{l}\text { Upper } \\
\text { conf. }\end{array}$} \\
\hline & & & & & & & & & & & \\
\hline $\mathrm{N}$ & 35 & 35 & & \multicolumn{2}{|l|}{26} & & 26 & 35 & 35 & 35 \\
\hline Min & 3 & 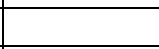 & 35 & \multicolumn{2}{|l|}{2} & \multicolumn{2}{|l|}{26} & & 4 & & \\
\hline Max & 980 & & & 764 & & & & & 928 & & \\
\hline Sum & 5368 & 2471 & 7839 & 468 & & 2757 & & 6340 & 5401 & 2596 & 7765 \\
\hline Mean & 153.37 & 70.60 & 223.97 & 180 & .27 & 106. & & 243.85 & 154.31 & 74.17 & 221.86 \\
\hline Std. error & 40.23 & 18.29 & 53.24 & 35. & & 15.3 & & 47.72 & 38.42 & 15.67 & 51.99 \\
\hline Variance & 56654.36 & 11708.75 & 99212.28 & 336 & 42.44 & 6094 & & 59209.7 & 51660.81 & 8597.74 & 94621.38 \\
\hline $\begin{array}{l}\text { Stand. } \\
\text { dev }\end{array}$ & 238.02 & 157.30 & 357.32 & 183 & & 119. & & 276.98 & 227.29 & 146.81 & 361.31 \\
\hline Median & 48.00 & 7.00 & 65.00 & 127 & .00 & 47.5 & & 186.50 & 53.00 & -73.00 & 76.00 \\
\hline 25 prentil & 26.00 & 13.00 & 32.00 & 56. & & 6.25 & & 103.50 & 21.00 & 3.00 & 28.00 \\
\hline 75 prentil & 134.00 & -158.00 & 187.00 & 238 & .00 & 117. & & 324.00 & 210.00 & 156.00 & 349.00 \\
\hline Skewness & 2.32 & 0.74 & 3.35 & 1.8 & & 0.89 & & 3.26 & 2.38 & 1.09 & 3.79 \\
\hline Kurtosis & 4.67 & -7.56 & 9.21 & 3.5 & & -3.47 & & 8.16 & 5.33 & -6.19 & 11.35 \\
\hline $\begin{array}{l}\text { Geom. } \\
\text { mean }\end{array}$ & 66.02 & 30.98 & 88.21 & 94. & & 29.7 & & 136.80 & 63.55 & 27.25 & 86.90 \\
\hline $\begin{array}{l}\text { Coeff. } \\
\text { var }\end{array}$ & 155.19 & 122.40 & 191.85 & 101 & .75 & 76.8 & & 135.89 & 147.29 & 116.02 & 193.13 \\
\hline Holiday & & & & & & & & & & & \\
\hline $16-10$ & $\begin{array}{l}\text { Lower } \\
\text { conf. }\end{array}$ & $\begin{array}{l}\text { Upper } \\
\text { conf. }\end{array}$ & $18-10$ & & $\begin{array}{l}\text { Low } \\
\text { conf }\end{array}$ & & $\begin{array}{l}\mathrm{Up} \\
\mathrm{col}\end{array}$ & & $20-10$ & $\begin{array}{l}\text { Lower } \\
\text { conf. }\end{array}$ & Upper conf. \\
\hline 16 & 16 & 16 & 11 & & 11 & & 11 & & 45 & 45 & 45 \\
\hline 29 & & & 10 & & & & & & 2 & & \\
\hline 1325 & & & 899 & & & & & & 707 & & \\
\hline 5005 & 2354 & 7032 & 2915 & & 694 & & 47 & & 7104 & 4652 & 9217 \\
\hline 312.81 & 147.13 & 439.50 & 265.00 & & 63.0 & & 43 & & 157.87 & 103.38 & 204.82 \\
\hline 78.68 & 0.00 & 106.18 & 98.17 & & 63.4 & & & & 26.52 & 16.46 & 33.65 \\
\hline 99059.50 & -26967.00 & 180382.7 & 106014 & .40 & 4422 & 3.11 & 20 & 346.80 & 31639.71 & 12186.74 & 50957.39 \\
\hline 314.74 & 155.04 & 496.30 & 325.60 & & 241. & & & & 177.88 & 129.71 & 244.75 \\
\hline 286.00 & 191.00 & 495.00 & 165.00 & & -101 & & 29 & & 100.00 & 79.00 & 126.00 \\
\hline 67.25 & -143.50 & 94.50 & 39.00 & & -87.0 & & 68 & & 42.50 & 11.00 & 66.00 \\
\hline 400.50 & 274.00 & 507.00 & 431.00 & & -37.0 & & 69 & & 204.50 & 89.00 & 289.00 \\
\hline 2.34 & 1.83 & 5.28 & 1.46 & & -0.09 & & 2.9 & & 1.89 & 1.02 & 2.65 \\
\hline 7.22 & 4.68 & 16.24 & 0.75 & & -7.99 & & 3.7 & & 3.18 & -2.68 & 6.22 \\
\hline 196.86 & 69.66 & 275.49 & 120.96 & & -29.0 & & & & 77.88 & 39.62 & 105.51 \\
\hline 100.62 & 69.50 & 151.94 & 122.87 & & 76.1 & & & & 112.67 & 92.19 & 137.14 \\
\hline
\end{tabular}


Ottosen et al, 2020. Genet. Biodiv. J, Special issue (Behavioural Instability), 1-60

\begin{tabular}{|c|c|c|c|c|c|c|c|c|c|c|c|c|}
\hline \multicolumn{10}{|l|}{ Control } & & & \\
\hline & $30-09$ & $\begin{array}{l}\text { Lower } \\
\text { conf. }\end{array}$ & $\begin{array}{l}\text { Upper } \\
\text { conf. }\end{array}$ & \multicolumn{2}{|c|}{$02-10$} & \multicolumn{2}{|c|}{$\begin{array}{l}\text { Lower } \\
\text { conf. }\end{array}$} & \multicolumn{2}{|l|}{$\begin{array}{l}\text { Upper } \\
\text { conf. }\end{array}$} & $05-10$ & $\begin{array}{l}\text { Lower } \\
\text { conf. }\end{array}$ & $\begin{array}{l}\text { Upper } \\
\text { conf. }\end{array}$ \\
\hline $\mathrm{N}$ & 16 & 16 & 16 & \multicolumn{2}{|l|}{14} & & & 12 & 12 & 12 \\
\hline Min & 2 & & & \multicolumn{2}{|c|}{5} & & 14 & \multicolumn{2}{|l|}{14} & 3 & & \\
\hline Max & 94 & & & \multicolumn{2}{|c|}{1114} & & & & 129 & & \\
\hline Sum & 451 & 256 & 613 & 140 & & -87 & & & & 315 & 59 & 481 \\
\hline Mean & 28.19 & 16.00 & 38.31 & 100 & & -62 & & 186.21 & & 26.25 & 4.92 & 40.08 \\
\hline Std. error & 5.89 & 1.58 & 7.76 & 78.2 & & 0.0 & & 110.55 & & 9.84 & 0.00 & 13.69 \\
\hline Variance & 555.10 & 40.06 & 962.61 & 856 & 5.60 & -4 & 5.59 & 171098 & 50 & 1160.93 & -471.65 & 2250.62 \\
\hline $\begin{array}{l}\text { Stand. } \\
\text { dev }\end{array}$ & 23.56 & 14.41 & 34.97 & 292 & & 116 & & 574.55 & & 34.07 & 15.29 & 59.70 \\
\hline Median & 19.00 & -7.00 & 23.00 & 12.5 & & -12 & & 16.50 & & 19.00 & 6.50 & 31.50 \\
\hline 25 prentil & 12.75 & 7.00 & 20.50 & 7.00 & & 1.0 & & 8.25 & & 5.75 & -7.50 & 8.00 \\
\hline 75 prentil & 45.00 & 37.00 & 70.00 & 42.5 & & -25 & & 70.00 & & 31.75 & -41.25 & 44.50 \\
\hline Skewness & 1.55 & 0.62 & 3.16 & 3.7 & & 3.6 & & 6.75 & & 2.88 & 2.61 & 6.24 \\
\hline Kurtosis & 2.90 & -1.32 & 7.55 & 13.8 & & 13. & & 28.93 & & 9.10 & 7.83 & 20.17 \\
\hline $\begin{array}{l}\text { Geom. } \\
\text { mean }\end{array}$ & 19.73 & 9.05 & 27.04 & 20. & & -5.5 & & 30.06 & & 15.63 & 3.34 & 22.21 \\
\hline $\begin{array}{l}\text { Coeff. } \\
\text { var }\end{array}$ & 83.58 & 58.95 & 115.16 & 291 & & 264 & & 514.40 & & 129.80 & 97.41 & 212.67 \\
\hline Holiday & & & & & & & & & & & & \\
\hline $16-10$ & $\begin{array}{l}\text { Lower } \\
\text { conf. }\end{array}$ & $\begin{array}{l}\text { Upper } \\
\text { conf. }\end{array}$ & $18-10$ & & $\begin{array}{l}\text { Low } \\
\text { conf }\end{array}$ & & $\begin{array}{l}\text { Up } \\
\text { cor }\end{array}$ & & 20 & -10 & $\begin{array}{l}\text { Lower } \\
\text { conf. }\end{array}$ & Upper conf. \\
\hline 14 & 14 & 14 & 20 & & 20 & & 20 & & 30 & & 30 & 30 \\
\hline 4 & & & 1 & & & & & & 1 & & & \\
\hline 30 & & & 536 & & & & & & 14 & & & \\
\hline 197 & 142 & 246 & 2793 & & 1323 & & 405 & & 24 & & -781 & 4361 \\
\hline 14.07 & 10.14 & 17.57 & 139.65 & & 66.1 & & 20 & & 82 & 63 & -26.03 & 145.37 \\
\hline 1.98 & 1.17 & 2.61 & 35.87 & & 20.4 & & 47. & & 49. & 04 & 0.00 & 69.13 \\
\hline 55.15 & 19.13 & 95.61 & 25726.8 & & 8387 & & 442 & 76.94 & & 154.59 & -52948.03 & 143361.40 \\
\hline 7.43 & 5.30 & 11.02 & 160.40 & & 113. & & 230 & .08 & & 8.62 & 93.10 & 506.45 \\
\hline 13.00 & 9.50 & 18.00 & 69.50 & & -66.5 & & & 00 & 10 & 50 & -5.00 & 15.00 \\
\hline 7.75 & 2.50 & 10.00 & 20.75 & & -7.00 & & 36. & & 5.0 & & 2.00 & 8.00 \\
\hline 18.00 & 8.25 & 23.00 & 228.00 & & 3.25 & & & .00 & 29 & 25 & -63.50 & 41.75 \\
\hline 0.92 & 0.10 & 2.41 & 1.45 & & 0.48 & & 2.5 & & 5.1 & & 4.84 & 8.72 \\
\hline 0.63 & -3.44 & 2.86 & 1.55 & & -3.56 & & 4.7 & & 27 & 03 & 24.89 & 53.19 \\
\hline 12.29 & 8.41 & 15.33 & 56.77 & & -0.26 & & 86. & & & 43 & 2.51 & 20.72 \\
\hline 52.77 & 38.05 & 74.81 & 114.86 & & 77.9 & & & 36 & & 5.07 & 256.10 & 517.17 \\
\hline
\end{tabular}


Ottosen et al, 2020. Genet. Biodiv. J, Special issue (Behavioural Instability), 1-60

\section{Sebastian}

\section{Passive}

\begin{tabular}{|c|c|c|c|c|c|c|c|c|c|c|c|c|c|}
\hline \multirow{2}{*}{ Control } & & & & & & & & & & & & & \\
\hline & $30-09$ & $\begin{array}{l}\text { Lower } \\
\text { conf. }\end{array}$ & \multicolumn{2}{|c|}{$\begin{array}{l}\text { Upper } \\
\text { conf. }\end{array}$} & \multicolumn{2}{|c|}{$02-10$} & \multicolumn{2}{|c|}{$\begin{array}{l}\text { Lower } \\
\text { conf. }\end{array}$} & \multicolumn{2}{|l|}{$\begin{array}{l}\text { Upper } \\
\text { conf. }\end{array}$} & $05-10$ & $\begin{array}{l}\text { Lower } \\
\text { conf. }\end{array}$ & $\begin{array}{l}\text { Upper } \\
\text { conf. }\end{array}$ \\
\hline $\mathrm{N}$ & 50 & 50 & \multicolumn{2}{|c|}{50} & \multicolumn{2}{|l|}{34} & \multicolumn{2}{|c|}{34} & \multicolumn{2}{|l|}{34} & 78 & 78 & 78 \\
\hline Min & 10 & & & & 7 & & & & & & 10 & & \\
\hline Max & 2002 & & & & 456 & & & & & & 2434 & & \\
\hline Sum & 11144 & 4939 & \multicolumn{2}{|c|}{16150} & \multicolumn{2}{|c|}{13365} & \multicolumn{2}{|c|}{1843} & \multicolumn{2}{|l|}{22356} & 10978 & 4487 & 15996 \\
\hline Mean & 222.88 & 98.78 & \multicolumn{2}{|c|}{323.00} & \multicolumn{2}{|c|}{393.09} & \multicolumn{2}{|c|}{54.21} & \multicolumn{2}{|l|}{657.53} & 140.74 & 57.53 & 205.08 \\
\hline Std. error & 57.38 & 16.45 & 75 . & & 161 & & 0.0 & & 224.78 & & 38.77 & 0.00 & 52.82 \\
\hline Variance & 164640.90 & 13522.72 & & 617.30 & 884 & 76.90 & $\begin{array}{l}- \\
249 \\
\end{array}$ & 8.80 & $\begin{array}{l}171789 \\
0\end{array}$ & & 117224.70 & $\begin{array}{l}- \\
35712.43 \\
\end{array}$ & 217619.80 \\
\hline $\begin{array}{l}\text { Stand. } \\
\text { dev }\end{array}$ & 405.76 & 249.59 & 59 & .83 & 940 & & 460 & & 1654.3 & & 342.38 & 164.99 & 555.03 \\
\hline Median & 44.00 & 21.50 & 56. & & 36. & & -32 & & 45.00 & & 33.00 & 24.00 & 41.00 \\
\hline 25 prentil & 25.75 & 17.50 & 30 & & 21. & & 13.5 & & 28.75 & & 18.00 & 14.00 & 24.00 \\
\hline 75 prentil & 231.75 & -25.50 & & .50 & 244 & & -36 & & 409.50 & & 85.75 & -2.50 & 121.50 \\
\hline Skewness & 2.77 & 1.40 & 3.8 & & 3.5 & & 1.8 & & 5.20 & & 4.88 & 3.06 & 7.17 \\
\hline Kurtosis & 8.08 & -3.22 & 14 & & 13. & & -2.7 & & 23.82 & & 27.92 & 4.78 & 49.51 \\
\hline $\begin{array}{l}\text { Geom. } \\
\text { mean }\end{array}$ & 72.49 & 37.36 & 94 & & 75. & & 18.2 & & 107.00 & & 45.25 & 29.67 & 56.13 \\
\hline $\begin{array}{l}\text { Coeff. } \\
\text { var }\end{array}$ & 182.05 & 138.58 & & .55 & 239 & & 162 & & 326.49 & & 243.27 & 187.51 & 330.72 \\
\hline Holiday & & & & & & & & & & & & & \\
\hline $16-10$ & $\begin{array}{l}\text { Lower } \\
\text { conf. }\end{array}$ & $\begin{array}{l}\text { Upper } \\
\text { conf. }\end{array}$ & & $18-10$ & & $\begin{array}{l}\text { Lower } \\
\text { conf. }\end{array}$ & & $\mathrm{Upp}$ & er conf. & 20 & & $\begin{array}{l}\text { Lower } \\
\text { conf. }\end{array}$ & Upper conf. \\
\hline 53 & 53 & 53 & & 78 & & 78 & & 78 & & 64 & & 64 & 64 \\
\hline 6 & & & & 3 & & & & & & 3 & & & \\
\hline 2510 & & & & 1835 & & & & & & 23 & & & \\
\hline 10082 & 2693 & 15735 & & 14344 & & 7854 & & 199 & & 11 & & 3913 & 17848 \\
\hline 190.23 & 50.81 & 296.89 & & 183.90 & & 100.6 & & 256 & & 18 & .77 & 61.14 & 278.88 \\
\hline 62.78 & 0.00 & 84.73 & & 40.36 & & 19.20 & & 52.5 & & 55. & & 0.00 & 75.18 \\
\hline 208895.30 & -32555.97 & 380498 & & 127087 & & 28761 & & 215 & 23.10 & & 584.60 & -10502.49 & 361692.40 \\
\hline 457.05 & 243.02 & 720.99 & & 356.49 & & 238.2 & & 516 & & 44 & 63 & 252.76 & 702.90 \\
\hline 40.00 & 30.00 & 51.00 & & 53.50 & & 42.50 & & 72.1 & & 35 . & & 23.00 & 45.00 \\
\hline 21.50 & 14.00 & 27.00 & & 20.00 & & 8.25 & & 24.0 & & 18. & & 11.75 & 22.50 \\
\hline 89.50 & -95.50 & 129.00 & & 201.00 & & 134.00 & & 329 & & 86. & & 44.00 & 122.25 \\
\hline 3.68 & 1.42 & 5.20 & & 3.23 & & 1.72 & & 4.23 & & 3.7 & & 1.69 & 5.05 \\
\hline 14.29 & -10.39 & 25.06 & & 10.65 & & -5.35 & & 17.2 & & 14 & & -7.63 & 24.30 \\
\hline 52.37 & 27.97 & 68.05 & & 59.64 & & 36.89 & & 75.9 & & 46 & & 25.41 & 59.67 \\
\hline 240.27 & 179.62 & 304.44 & & 193.85 & & 160.4 & & 234 & & & .83 & 184.37 & 303.93 \\
\hline
\end{tabular}


Ottosen et al, 2020. Genet. Biodiv. J, Special issue (Behavioural Instability), 1-60

\begin{tabular}{|c|c|c|c|c|c|c|c|c|c|c|}
\hline \multirow{2}{*}{\multicolumn{11}{|c|}{ Covement }} \\
\hline & \multirow[b]{2}{*}{$30-09$} & \multirow[b]{2}{*}{$\begin{array}{l}\text { Lower } \\
\text { conf. }\end{array}$} & \multirow[b]{2}{*}{$\begin{array}{l}\text { Upper } \\
\text { conf. }\end{array}$} & \multirow{2}{*}{\multicolumn{2}{|c|}{$02-10$}} & \multirow[b]{2}{*}{$\begin{array}{l}\text { Lower } \\
\text { conf. }\end{array}$} & & & & \multirow[b]{2}{*}{$\begin{array}{l}\text { Upper } \\
\text { conf. }\end{array}$} \\
\hline & & & & & & & $\begin{array}{l}\text { Upper } \\
\text { conf. }\end{array}$ & $05-10$ & $\begin{array}{l}\text { Lower } \\
\text { conf. }\end{array}$ & \\
\hline $\mathrm{N}$ & 89 & 89 & 89 & 73 & & 73 & 73 & 154 & 154 & 154 \\
\hline Min & 2 & & & 3 & & & & 2 & & \\
\hline Max & 125 & & & 122 & & & & 325 & & \\
\hline Sum & 2245 & 1801 & 2652 & 1701 & & 1346 & 2016 & 3771 & 2813 & 4547 \\
\hline Mean & 25.22 & 20.24 & 29.80 & 23.30 & & 18.44 & 27.62 & 24.49 & 18.27 & 29.53 \\
\hline Std. error & 2.47 & 1.63 & 3.00 & 2.42 & & 1.29 & 3.04 & 2.91 & 0.00 & 3.80 \\
\hline Variance & 542.88 & 236.68 & 802.65 & 428.16 & & 122.29 & 674.72 & 1304.27 & -102.85 & 2221.25 \\
\hline $\begin{array}{l}\text { Stand. } \\
\text { dev }\end{array}$ & 23.30 & 17.46 & 29.77 & 20.69 & & 14.29 & 27.91 & 36.11 & 20.16 & 52.55 \\
\hline Median & 18.00 & 14.00 & 22.00 & 18.00 & & 14.00 & 22.00 & 15.00 & 12.00 & 18.00 \\
\hline 25 prentil & 9.00 & 6.50 & 10.00 & 10.00 & & 7.00 & 13.00 & 7.75 & 6.50 & 9.50 \\
\hline 75 prentil & 32.50 & 20.00 & 40.00 & 29.00 & & 19.00 & 35.00 & 26.00 & 19.00 & 29.00 \\
\hline Skewness & 2.01 & 1.39 & 2.90 & 2.38 & & 1.58 & 3.71 & 5.11 & 3.67 & 8.23 \\
\hline Kurtosis & 4.87 & 0.69 & 9.17 & 7.49 & & 0.94 & 14.53 & 34.86 & 12.31 & 65.50 \\
\hline $\begin{array}{l}\text { Geom. } \\
\text { mean }\end{array}$ & 17.45 & 13.96 & 20.33 & 17.20 & & 13.87 & 19.94 & 14.57 & 12.15 & 16.61 \\
\hline $\begin{array}{l}\text { Coeff. } \\
\text { var }\end{array}$ & 92.37 & 78.13 & 109.85 & 88.80 & & 71.87 & 111.42 & 147.48 & 113.48 & 199.22 \\
\hline \multicolumn{11}{|l|}{ Holiday } \\
\hline $16-10$ & $\begin{array}{l}\text { Lower } \\
\text { conf. }\end{array}$ & $\begin{array}{l}\text { Upper } \\
\text { conf. }\end{array}$ & $18-10$ & & $\begin{array}{l}\text { Lower } \\
\text { conf. }\end{array}$ & & $\begin{array}{l}\text { Upper } \\
\text { conf. }\end{array}$ & $20-10$ & $\begin{array}{l}\text { Lower } \\
\text { conf. }\end{array}$ & Upper conf. \\
\hline 115 & 115 & 115 & 145 & & 145 & & 145 & 132 & 132 & 132 \\
\hline 1 & & & 2 & & & & & 3 & & \\
\hline 195 & & & 114 & & & & & 189 & & \\
\hline 2814 & 2122 & 3398 & 2897 & & 2400 & & 3362 & 3268 & 2620 & 3832 \\
\hline 24.47 & 18.45 & 29.55 & 19.98 & & 16.55 & & 23.19 & 24.76 & 19.85 & 29.03 \\
\hline 2.84 & 1.39 & 3.58 & 1.70 & & 1.32 & & 1.97 & 2.35 & 1.26 & 2.93 \\
\hline 929.16 & 222.65 & 1477.59 & 418.52 & & 253.68 & & 563.00 & 731.80 & 209.55 & 1132.97 \\
\hline 30.48 & 20.52 & 41.45 & 20.46 & & 16.76 & & 24.36 & 27.05 & 18.69 & 35.92 \\
\hline 14.00 & 10.00 & 17.00 & 12.00 & & 9.00 & & 15.00 & 15.00 & 10.00 & 18.00 \\
\hline 7.00 & 5.00 & 8.00 & 7.00 & & 6.00 & & 9.00 & 8.00 & 6.00 & 9.00 \\
\hline 29.00 & 20.00 & 34.00 & 26.00 & & 18.00 & & 33.50 & 30.75 & 21.50 & 35.50 \\
\hline 3.14 & 2.29 & 4.51 & 1.85 & & 1.27 & & 2.43 & 3.10 & 2.33 & 4.89 \\
\hline 12.28 & 3.37 & 21.41 & 3.48 & & -0.23 & & 6.42 & 13.53 & 5.46 & 25.98 \\
\hline 14.60 & 11.62 & 17.03 & 12.91 & & 10.76 & & 14.74 & 16.23 & 13.49 & 18.51 \\
\hline 124.57 & 104.19 & 154.61 & 102.39 & & 90.80 & & 114.30 & 109.27 & 89.07 & 135.86 \\
\hline
\end{tabular}


Ottosen et al, 2020. Genet. Biodiv. J, Special issue (Behavioural Instability), 1-60

\begin{tabular}{|c|c|c|c|c|c|c|c|c|c|c|c|}
\hline \multicolumn{12}{|c|}{ Foraging } \\
\hline \multirow[t]{2}{*}{ Control } & \multirow[b]{2}{*}{$30-09$} & \multirow[b]{2}{*}{$\begin{array}{l}\text { Lower } \\
\text { conf. }\end{array}$} & \multirow[b]{2}{*}{$\begin{array}{l}\text { Upper } \\
\text { conf. }\end{array}$} & \multirow{2}{*}{\multicolumn{2}{|c|}{$02-10$}} & \multirow{2}{*}{\multicolumn{2}{|c|}{$\begin{array}{l}\text { Lower } \\
\text { conf. }\end{array}$}} & \multirow[b]{2}{*}{$\begin{array}{l}\text { Upper } \\
\text { conf. }\end{array}$} & \multirow[b]{2}{*}{$05-10$} & \multirow[b]{2}{*}{$\begin{array}{l}\text { Lower } \\
\text { conf. }\end{array}$} & \multirow[b]{2}{*}{$\begin{array}{l}\text { Upper } \\
\text { conf. }\end{array}$} \\
\hline & & & & & & & & & & & \\
\hline $\mathrm{N}$ & 46 & 46 & 46 & \multicolumn{2}{|l|}{29} & & & 36 & 36 & 36 \\
\hline Min & 2 & & & \multicolumn{2}{|l|}{3} & \multicolumn{2}{|l|}{29} & 29 & 1 & & \\
\hline Max & 600 & & & \multicolumn{2}{|c|}{549} & & & 1012 & & \\
\hline Sum & 4230 & 2098 & 6068 & 491 & & & & 6817 & 5741 & 2676 & 8421 \\
\hline Mean & 91.96 & 45.61 & 131.91 & 169 & .34 & 100.4 & & 235.07 & 159.47 & 74.33 & 233.92 \\
\hline Std. error & 22.31 & 12.38 & 28.99 & 35. & & 28.27 & & 43.74 & 41.23 & 17.21 & 55.72 \\
\hline Variance & 22902.84 & 7051.23 & 38669.10 & 366 & 21.52 & 23180 & 0.77 & 55487.5 & 61185.28 & 10665.67 & 111779.20 \\
\hline $\begin{array}{l}\text { Stand. } \\
\text { dev }\end{array}$ & 151.34 & 105.81 & 218.20 & 191 & .37 & 158.9 & & 249.49 & 247.36 & 160.49 & 391.80 \\
\hline Median & 31.50 & 16.50 & 41.00 & 83. & & -9.00 & & 132.00 & 62.50 & 5.00 & 96.00 \\
\hline 25 prentil & 17.25 & 9.50 & 29.50 & 20. & & -10.50 & & 29.00 & 16.25 & -8.50 & 26.50 \\
\hline 75 prentil & 69.50 & -49.75 & 96.00 & 256 & .50 & -8.00 & & 389.00 & 173.50 & 31.00 & 256.00 \\
\hline Skewness & 2.35 & 0.79 & 3.26 & 1.1 & & 0.12 & & 1.81 & 2.45 & 1.07 & 3.64 \\
\hline Kurtosis & 4.64 & -7.56 & 8.73 & -0.2 & & -4.70 & & 1.10 & 5.70 & -6.43 & 11.19 \\
\hline $\begin{array}{l}\text { Geom. } \\
\text { mean }\end{array}$ & 32.64 & 15.22 & 43.99 & 73. & & 23.29 & & 103.83 & 52.56 & 14.69 & 75.35 \\
\hline $\begin{array}{l}\text { Coeff. } \\
\text { var }\end{array}$ & 164.57 & 132.49 & 198.05 & 113 & .00 & 83.33 & & 138.64 & 155.11 & 119.74 & 200.58 \\
\hline Holiday & & & & & & & & & & & \\
\hline $16-10$ & $\begin{array}{l}\text { Lower } \\
\text { conf. }\end{array}$ & $\begin{array}{l}\text { Upper } \\
\text { conf. }\end{array}$ & $18-10$ & & $\begin{array}{l}\text { Lowe } \\
\text { conf. }\end{array}$ & & $\begin{array}{l}\text { Upp } \\
\text { cont }\end{array}$ & & $20-10$ & Lower conf. & Upper conf. \\
\hline 28 & 28 & 28 & 23 & & 23 & & 23 & & 42 & 42 & 42 \\
\hline 2 & & & 1 & & & & & & 2 & & \\
\hline 937 & & & 600 & & & & & & 875 & & \\
\hline 4061 & 1829 & 5790 & 2710 & & 1295 & & 391 & & 6632 & 3467 & 9469 \\
\hline 145.04 & 65.32 & 206.79 & 117.83 & & 56.30 & & 170 & & 157.90 & 82.55 & 225.45 \\
\hline 36.94 & 0.00 & 50.24 & 29.76 & & 3.58 & & 39.0 & & 36.86 & 25.74 & 45.75 \\
\hline 38203.22 & -7733.66 & 70671.03 & 20373.2 & & 294.4 & & 350 & 18.77 & 57066.67 & 27823.99 & 87908.85 \\
\hline 195.46 & 100.84 & 315.18 & 142.73 & & 84.34 & & 209 & & 238.89 & 183.99 & 315.83 \\
\hline 67.50 & -15.50 & 95.00 & 82.00 & & 53.00 & & 145 & & 22.00 & -29.50 & 32.00 \\
\hline 36.50 & 26.00 & 53.00 & 7.00 & & -39.0 & & 12.0 & & 8.25 & 3.50 & 12.50 \\
\hline 193.00 & 128.50 & 267.00 & 190.00 & & 111.0 & & 294 & & 231.50 & 19.00 & 409.25 \\
\hline 2.92 & 1.91 & 5.30 & 1.96 & & 1.08 & & 3.64 & & 1.58 & 0.57 & 2.31 \\
\hline 10.05 & 2.14 & 21.26 & 4.86 & & -0.03 & & 11.1 & & 1.44 & -3.72 & 3.67 \\
\hline 72.13 & 29.96 & 99.87 & 39.32 & & -2.76 & & 60.9 & & 34.53 & 6.88 & 49.76 \\
\hline 134.76 & 100.87 & 191.88 & 121.14 & & 82.05 & & 162 & & 151.29 & 105.28 & 185.33 \\
\hline
\end{tabular}


Ottosen et al, 2020. Genet. Biodiv. J, Special issue (Behavioural Instability), 1-60

\begin{tabular}{|c|c|c|c|c|c|c|c|c|c|c|c|c|}
\hline \multicolumn{13}{|l|}{ Interaction } \\
\hline \multirow[t]{2}{*}{ Control } & & & & & & & & & & & & \\
\hline & $30-09$ & \multicolumn{2}{|c|}{ Lower conf. } & $\begin{array}{l}\text { Upper } \\
\text { conf. }\end{array}$ & $02-10$ & \multicolumn{2}{|c|}{ Lower conf. } & \multicolumn{2}{|c|}{$\begin{array}{l}\text { Upper } \\
\text { conf. }\end{array}$} & $05-10$ & $\begin{array}{l}\text { Lower } \\
\text { conf. }\end{array}$ & $\begin{array}{l}\text { Upper } \\
\text { conf. }\end{array}$ \\
\hline $\mathrm{N}$ & 27 & \multicolumn{2}{|l|}{27} & 27 & 16 & \multicolumn{2}{|l|}{16} & & 27 & 27 & 27 \\
\hline Min & 6 & & & 3 & & & 16 & 4 & & \\
\hline Max & 132 & & & & 626 & \multirow{2}{*}{\multicolumn{2}{|c|}{-350}} & & 72 & & \\
\hline Sum & 755 & \multicolumn{2}{|l|}{431} & 1012 & 919 & & & 1612 & & 509 & 335 & 658 \\
\hline Mean & 27.96 & \multicolumn{2}{|l|}{15.96} & 37.48 & 57.44 & \multicolumn{2}{|l|}{-21.88} & \multicolumn{2}{|c|}{100.75} & 18.85 & 12.41 & 24.37 \\
\hline Std. error & 5.62 & \multicolumn{2}{|l|}{0.00} & 7.72 & 38.08 & \multicolumn{2}{|l|}{0.00} & \multicolumn{2}{|c|}{53.79} & 3.23 & 1.42 & 4.31 \\
\hline Variance & 851.96 & \multicolumn{2}{|l|}{-11.86} & 1608.36 & 23207.06 & \multicolumn{2}{|l|}{-14326.53} & \multicolumn{2}{|c|}{46286.90} & 281.13 & 54.35 & 501.49 \\
\hline $\begin{array}{l}\text { Stand. } \\
\text { dev }\end{array}$ & 29.19 & \multicolumn{2}{|l|}{16.95} & 48.60 & 152.34 & \multicolumn{2}{|l|}{58.22} & 293. & & 16.77 & 11.00 & 25.74 \\
\hline Median & 20.00 & 13.00 & & 28.00 & 17.00 & 1.00 & & 24.0 & & 14.00 & 8.00 & 19.00 \\
\hline 25 prentil & 11.00 & 6.00 & & 13.00 & 6.25 & -3.50 & & 9.50 & & 9.00 & 8.00 & 12.00 \\
\hline 75 prentil & 35.00 & -3.00 & & 50.00 & 40.50 & 36.00 & & 63.5 & & 25.00 & 21.00 & 34.00 \\
\hline Skewness & 2.52 & 1.09 & & 4.40 & 3.94 & 3.90 & & 7.81 & & 1.97 & 0.96 & 3.34 \\
\hline Kurtosis & 6.50 & -5.10 & & 13.71 & 15.63 & 15.46 & & 33.0 & & 3.83 & -3.82 & 8.52 \\
\hline $\begin{array}{l}\text { Geom. } \\
\text { mean }\end{array}$ & 19.93 & 13.08 & & 24.84 & 17.27 & 0.68 & & 24.9 & & 13.92 & 9.28 & 17.32 \\
\hline $\begin{array}{l}\text { Coeff. } \\
\text { var }\end{array}$ & 104.38 & 84.87 & & 156.85 & 265.23 & 235.53 & & 470. & & 88.94 & 71.39 & 122.44 \\
\hline Holiday & & & & & & & & & & & & \\
\hline $16-10$ & & $\begin{array}{l}\text { Lower } \\
\text { conf. }\end{array}$ & $\begin{array}{l}\text { Upper } \\
\text { conf. }\end{array}$ & $18-10$ & & $\begin{array}{l}\text { Lower } \\
\text { conf. }\end{array}$ & $\begin{array}{l}\text { Upp } \\
\text { con }\end{array}$ & & $20-1$ & & $\begin{array}{l}\text { Lower } \\
\text { conf. }\end{array}$ & $\begin{array}{l}\text { Upper } \\
\text { conf. }\end{array}$ \\
\hline 17 & & 17 & 17 & 17 & & 17 & 17 & & 37 & & 37 & 37 \\
\hline 3 & & & & 1 & & & & & 1 & & & \\
\hline 81 & & & & 170 & & & & & 690 & & & \\
\hline 386 & & 215 & 520 & 469 & & 101 & 743 & & 2068 & & 238 & 3420 \\
\hline 22.71 & & 12.65 & 30.59 & 27.59 & & 5.94 & 43.7 & & 55.8 & & 6.43 & 92.43 \\
\hline 4.73 & & 0.00 & 6.26 & 10.30 & & 0.00 & 14.2 & & 22.4 & & 0.00 & 31.45 \\
\hline 380.22 & & -13.32 & 666.33 & 1802.01 & & -459.49 & 343 & .79 & 187 & 8.88 & -6256.61 & 36606.12 \\
\hline 19.50 & & 11.18 & 29.30 & 42.45 & & 21.15 & 71.9 & & 136. & & 64.58 & 245.07 \\
\hline 14.00 & & -6.00 & 16.00 & 11.00 & & -5.00 & 19.0 & & 7.00 & & -5.00 & 8.00 \\
\hline 11.00 & & 8.50 & 18.00 & 2.50 & & -5.00 & 4.00 & & 4.50 & & 2.50 & 6.50 \\
\hline 35.00 & & 26.00 & 53.00 & 36.00 & & -3.00 & 58.5 & & 30.5 & & -26.00 & 47.00 \\
\hline 1.80 & & 0.98 & 3.61 & 2.69 & & 1.85 & 4.70 & & 3.65 & & 1.70 & 5.31 \\
\hline 4.07 & & 0.08 & 9.82 & 8.12 & & 2.87 & 17.3 & & 14.0 & & -4.72 & 25.27 \\
\hline 16.33 & & 8.42 & 21.67 & 10.28 & & -0.24 & 15.4 & & 12.5 & & 4.08 & 17.41 \\
\hline 85.88 & & 61.73 & 119.33 & 153.87 & & 105.11 & 220 & & 244. & & 170.25 & 342.31 \\
\hline
\end{tabular}


Ottosen et al, 2020. Genet. Biodiv. J, Special issue (Behavioural Instability), 1-60

\section{Laura}

Passive behaviour

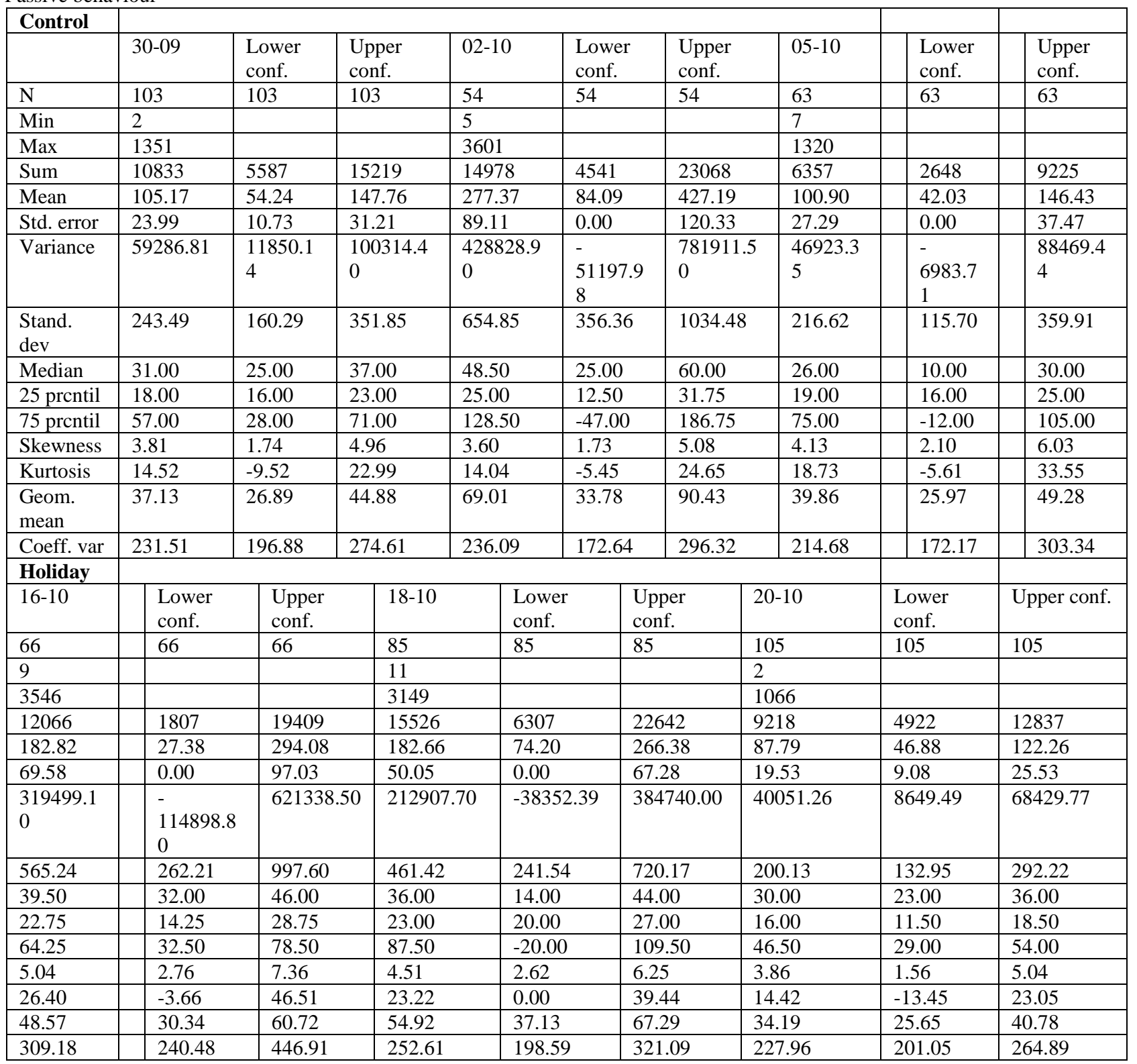


Ottosen et al, 2020. Genet. Biodiv. J, Special issue (Behavioural Instability), 1-60

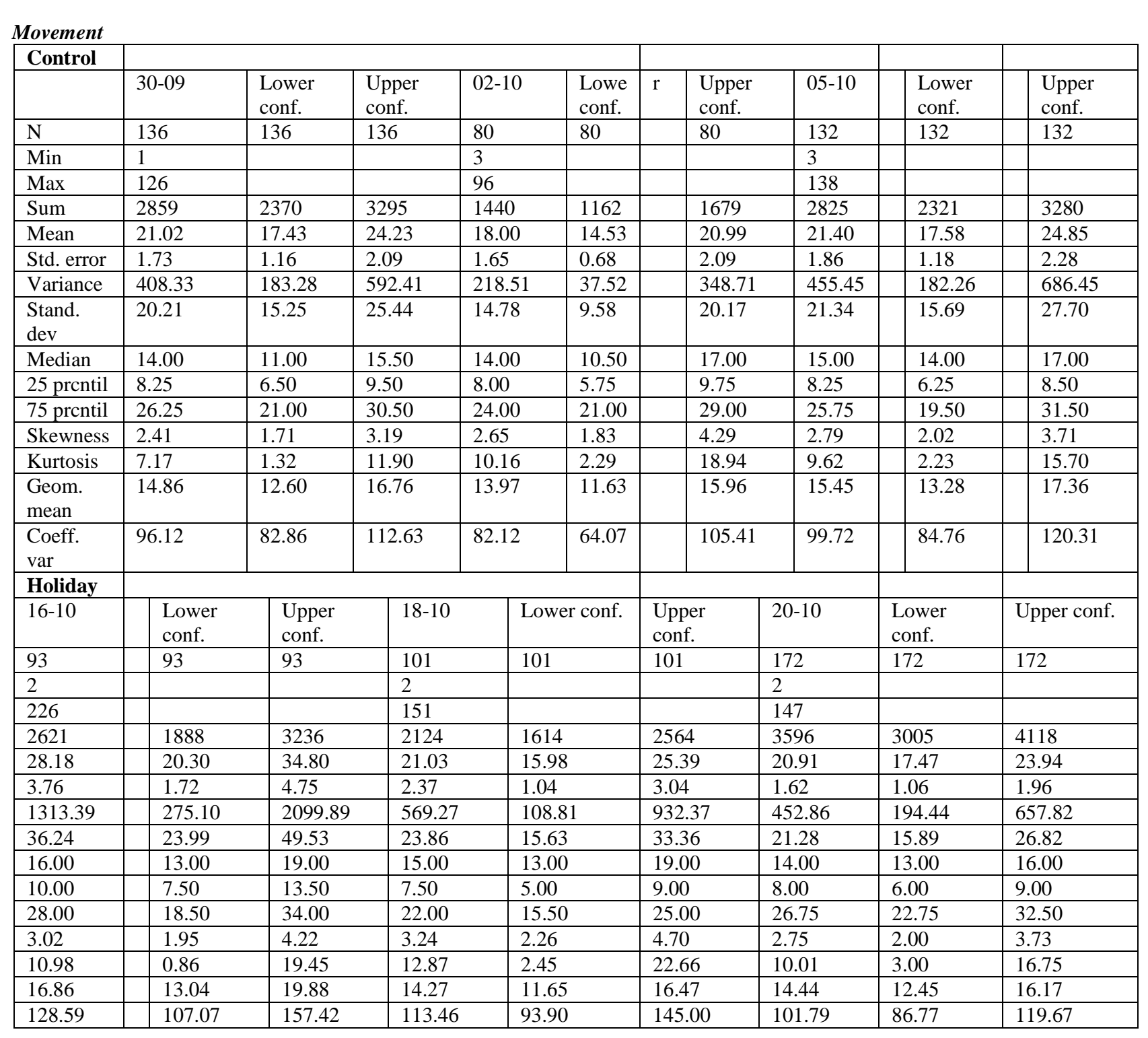


Ottosen et al, 2020. Genet. Biodiv. J, Special issue (Behavioural Instability), 1-60

\begin{tabular}{|c|c|c|c|c|c|c|c|c|c|c|c|}
\hline \multicolumn{12}{|l|}{ Foraging } \\
\hline \multicolumn{12}{|l|}{ Control } \\
\hline & $30-09$ & $\begin{array}{l}\text { Lower } \\
\text { conf. }\end{array}$ & $\begin{array}{l}\text { Upper } \\
\text { conf. }\end{array}$ & \multicolumn{2}{|c|}{$02-10$} & \multicolumn{2}{|c|}{$\begin{array}{l}\text { Lower } \\
\text { conf. }\end{array}$} & $\begin{array}{l}\text { Upper } \\
\text { conf. }\end{array}$ & $05-10$ & $\begin{array}{l}\text { Lower } \\
\text { conf. }\end{array}$ & $\begin{array}{l}\text { Upper } \\
\text { conf. }\end{array}$ \\
\hline $\mathrm{N}$ & 28 & \multicolumn{2}{|l|}{28} & \multicolumn{2}{|l|}{20} & & & 42 & 42 & 42 \\
\hline Min & 6 & & & \multicolumn{2}{|l|}{6} & \multicolumn{2}{|l|}{20} & 20 & 9 & & \\
\hline Max & 662 & & & \multicolumn{2}{|c|}{487} & & 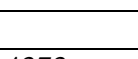 & 740 & & \\
\hline Sum & 3165 & 1421 & 4572 & 317 & & & & 4373 & 5192 & 2944 & 7038 \\
\hline Mean & 113.04 & 50.75 & 163.29 & 158. & & 88.60 & & 218.65 & 123.62 & 70.10 & 167.57 \\
\hline Std. error & 29.35 & 5.01 & 39.45 & 34.0 & & 24.26 & & 43.54 & 24.81 & 10.93 & 31.96 \\
\hline Variance & 24117.67 & 702.08 & 43577.29 & 231 & 31.25 & 1177 & 1.21 & 37921.59 & 25862.44 & 5013.41 & 42902.21 \\
\hline $\begin{array}{l}\text { Stand. } \\
\text { dev }\end{array}$ & 155.30 & 92.58 & 242.35 & 152 & & 118.5 & & 212.63 & 160.82 & 105.51 & 227.71 \\
\hline Median & 52.00 & -0.50 & 77.50 & 120 & & 63.50 & & 184.50 & 51.00 & 11.00 & 65.50 \\
\hline 25 prentil & 21.50 & 13.25 & 33.00 & 37.2 & & -16.2 & & 62.00 & 22.50 & 8.00 & 27.00 \\
\hline 75 prentil & 157.50 & 28.25 & 248.25 & 197 & & -45.0 & & 249.75 & 158.00 & 19.00 & 240.25 \\
\hline Skewness & 2.33 & 1.11 & 3.64 & 1.13 & & 0.12 & & 2.03 & 2.18 & 1.20 & 3.26 \\
\hline Kurtosis & 5.68 & -3.54 & 11.52 & 0.11 & & -5.51 & & 1.92 & 5.08 & -1.62 & 10.12 \\
\hline $\begin{array}{l}\text { Geom. } \\
\text { mean }\end{array}$ & 52.07 & 19.68 & 71.66 & 90.0 & & 27.89 & & 127.77 & 61.52 & 34.40 & 80.05 \\
\hline $\begin{array}{l}\text { Coeff. } \\
\text { var }\end{array}$ & 137.39 & 103.90 & 181.33 & 95.9 & & 70.59 & & 122.16 & 130.09 & 102.11 & 160.89 \\
\hline Holiday & & & & & & & & & & & \\
\hline $16-10$ & $\begin{array}{l}\text { Lower } \\
\text { conf. }\end{array}$ & $\begin{array}{l}\text { Upper } \\
\text { conf. }\end{array}$ & $\begin{array}{l}18- \\
10\end{array}$ & & $\begin{array}{l}\text { Lowe } \\
\text { conf. }\end{array}$ & & $\begin{array}{l}\text { Up1 } \\
\text { con }\end{array}$ & & $20-10$ & $\begin{array}{l}\text { Lower } \\
\text { conf. }\end{array}$ & Upper conf. \\
\hline 22 & 22 & 22 & 12 & & 12 & & 12 & & 49 & 49 & 49 \\
\hline 22 & & & 14 & & & & & & 1 & & \\
\hline 577 & & & 549 & & & & & & 1166 & & \\
\hline 3067 & 1389 & 4425 & 2285 & & 1018 & & 345 & & 5544 & 2472 & 7873 \\
\hline 139.41 & 63.14 & 201.14 & 190.42 & & 84.83 & & 287 & & 113.14 & 50.45 & 160.67 \\
\hline 36.64 & 19.60 & 50.54 & 54.18 & & 38.75 & & 70.2 & & 28.67 & 0.00 & 39.35 \\
\hline 29533.11 & 8453.51 & 56191.92 & 35226.8 & & 1802 & .17 & 591 & 3.36 & 40284.67 & -11362.04 & 75863.44 \\
\hline 171.85 & 118.73 & 290.09 & 187.69 & & 146.3 & & 269 & & 200.71 & 98.22 & 332.82 \\
\hline 69.00 & 9.00 & 98.00 & 87.50 & & -140. & & 129 & & 70.00 & 56.00 & 112.00 \\
\hline 38.25 & 29.50 & 51.75 & 44.25 & & -18.5 & & $74 .($ & & 11.50 & -8.00 & 16.00 \\
\hline 157.25 & -188.25 & 220.25 & 325.25 & & 123.0 & & 553 & & 118.50 & 80.50 & 153.00 \\
\hline 1.97 & 0.33 & 3.25 & 0.82 & & -0.62 & & 1.9 & & 3.95 & 2.43 & 6.47 \\
\hline 2.78 & -9.37 & 6.89 & -0.72 & & -6.25 & & 0.6 & & 17.64 & 1.20 & 33.40 \\
\hline 80.89 & 38.31 & 107.60 & 102.94 & & 4.26 & & 154 & & 42.42 & 20.44 & 57.34 \\
\hline 123.27 & 100.51 & 176.82 & 98.57 & & 58.19 & & 134 & & 177.40 & 141.32 & 259.07 \\
\hline
\end{tabular}


Ottosen et al, 2020. Genet. Biodiv. J, Special issue (Behavioural Instability), 1-60

Stereotype behaviour
\begin{tabular}{|l|l|l|l|l|l|l|}
\hline & Control & & & Holiday & & \\
\hline & $05-10$ & Lower conf. & Upper conf. & $20-10$ & Lower conf. & Upper conf. \\
\hline $\mathrm{N}$ & 6 & 6 & 6 & 6 & 6 & \\
\hline Min & 34 & & & 33 & & \\
\hline Max & 331 & & & 211 & & \\
\hline Sum & 758 & 234 & 1139 & 600 & 299 & 846 \\
\hline Mean & 126.33 & 39.00 & 189.83 & 100.00 & 49.83 & 141.00 \\
\hline Std. error & 43.43 & 16.78 & 60.59 & 25.59 & 11.50 & 35.33 \\
\hline Variance & 11314.67 & 1689.17 & 22027.57 & 3930.00 & 792.93 & 7488.90 \\
\hline Stand. dev & 106.37 & 68.03 & 188.21 & 62.69 & 41.31 & 106.12 \\
\hline Median & 109.00 & -8.50 & 174.50 & 86.00 & 3.50 & 126.50 \\
\hline 25 prcntil & 48.25 & -17.00 & 62.50 & 51.75 & 15.50 & 70.50 \\
\hline 75 prcntil & 174.25 & 17.50 & 244.00 & 147.25 & 83.50 & 210.50 \\
\hline Skewness & 1.84 & 1.28 & 5.72 & 1.22 & 0.28 & 3.69 \\
\hline Kurtosis & 3.90 & 1.98 & 11.08 & 1.71 & -1.84 & 6.46 \\
\hline Geom. mean & 97.49 & 25.77 & 139.55 & 84.96 & 36.01 & 116.97 \\
\hline Coeff. var & 84.20 & 57.74 & 143.27 & 62.69 & 40.01 & 103.86 \\
\hline
\end{tabular}

\section{Interaction}

\begin{tabular}{|c|c|c|c|c|c|c|c|c|c|}
\hline \multicolumn{10}{|l|}{ Control } \\
\hline & $30-09$ & $\begin{array}{l}\text { Lower } \\
\text { conf. }\end{array}$ & $\begin{array}{l}\text { Upper } \\
\text { conf. }\end{array}$ & $02-10$ & $\begin{array}{l}\text { Lower } \\
\text { conf. }\end{array}$ & $\begin{array}{l}\text { Upper } \\
\text { conf. }\end{array}$ & $05-10$ & $\begin{array}{l}\text { Lower } \\
\text { conf. }\end{array}$ & $\begin{array}{l}\text { Upper } \\
\text { conf. }\end{array}$ \\
\hline $\mathrm{N}$ & 14 & 14 & 14 & 11 & 11 & & 14 & 14 & 14 \\
\hline Min & 5 & & & 3 & & 11 & 9 & & \\
\hline Max & 319 & & & 1114 & & & 499 & & \\
\hline Sum & 702 & 29 & 1127 & 1371 & & 2541 & 1150 & 95 & 1857 \\
\hline Mean & 50.14 & 2.07 & 80.50 & 124.64 & -76.73 & 231.00 & 82.14 & 6.79 & 132.64 \\
\hline Std. error & 21.79 & 0.00 & 30.59 & 99.08 & 0.00 & 140.06 & 34.73 & 0.00 & 48.61 \\
\hline Variance & 6650.29 & -2966.60 & 13099.85 & 107983.10 & $\begin{array}{l}- \\
45199.91 \\
\end{array}$ & 215786.10 & 16888.75 & $\begin{array}{l}- \\
6417.61 \\
\end{array}$ & 33075.01 \\
\hline $\begin{array}{l}\text { Stand. } \\
\text { dev }\end{array}$ & 81.55 & 35.56 & 148.93 & 328.61 & 146.17 & 643.80 & 129.96 & 59.43 & 233.41 \\
\hline Median & 23.50 & 2.00 & 35.50 & 26.00 & 7.00 & 43.00 & 26.00 & -27.00 & 33.00 \\
\hline 25 prentil & 11.00 & -0.25 & 15.50 & 9.00 & -8.00 & 15.00 & 16.75 & 7.50 & 23.75 \\
\hline 75 prentil & 48.75 & -56.50 & 71.50 & 45.00 & -1024.00 & 64.00 & 85.50 & -96.25 & 145.00 \\
\hline Skewness & 3.16 & 2.75 & 5.87 & 3.30 & 3.29 & 7.20 & 2.93 & 2.32 & 5.31 \\
\hline Kurtosis & 10.68 & 8.26 & 22.72 & 10.92 & 10.87 & 23.79 & 9.23 & 5.67 & 19.86 \\
\hline $\begin{array}{l}\text { Geom. } \\
\text { mean }\end{array}$ & 25.22 & 4.82 & 35.73 & 25.44 & -14.92 & 39.86 & 39.29 & 5.34 & 56.52 \\
\hline $\begin{array}{l}\text { Coeff. } \\
\text { var }\end{array}$ & 162.63 & 124.61 & 263.17 & 263.65 & 243.44 & 478.88 & 158.21 & 115.81 & 246.18 \\
\hline Holiday & & & & & & & & & \\
\hline $16-10$ & $\begin{array}{l}\text { Lower } \\
\text { conf. }\end{array}$ & $\begin{array}{l}\text { Upper } \\
\text { conf. }\end{array}$ & $\begin{array}{l}18- \\
10 \\
\end{array}$ & $\begin{array}{l}\text { Lower } \\
\text { conf. }\end{array}$ & $\begin{array}{l}\mathrm{Up} \\
\mathrm{co}\end{array}$ & & -10 & $\begin{array}{l}\text { Lower } \\
\text { conf. }\end{array}$ & Upper conf. \\
\hline 13 & 13 & 13 & 14 & 14 & 14 & 3 & & 31 & 31 \\
\hline 3 & & & 5 & & & 1 & & & \\
\hline 108 & & & 538 & & & & & & \\
\hline 451 & 215 & 663 & \begin{tabular}{l|l}
1493 & \\
\end{tabular} & 331 & 24 & & & -745 & 5503 \\
\hline 34.69 & 16.54 & 51.00 & 106.64 & 23.64 & & & 2.42 & -24.03 & 177.52 \\
\hline 9.08 & 5.21 & 11.83 & 40.76 & 0.00 & 55 & & 85 & 0.00 & 81.63 \\
\hline 1072.90 & 353.23 & 1819.82 & 23253.7 & -860.6 & & 93.51 & 3740.80 & -74008.83 & 206589.00 \\
\hline 32.76 & 23.17 & 47.46 & 152.49 & 87.34 & & & 2.09 & 113.62 & 614.30 \\
\hline 25.00 & -2.00 & 42.00 & 34.50 & -70.00 & 50 & & .00 & 14.00 & 40.00 \\
\hline 6.50 & -12.00 & 10.00 & 17.75 & -1.25 & 29 & 9. & & 3.00 & 16.00 \\
\hline 60.50 & 30.50 & 95.50 & 211.75 & 96.25 & & & .00 & -23.00 & 86.00 \\
\hline 1.04 & -0.03 & 2.18 & 2.05 & 0.73 & 3.7 & 5 & & 4.74 & 8.57 \\
\hline 0.41 & -4.10 & 2.65 & 4.31 & -3.36 & 10 & & .25 & 24.06 & 52.56 \\
\hline 19.86 & 2.67 & 29.55 & 43.64 & -0.82 & 65 & & .45 & 4.50 & 30.60 \\
\hline 94.42 & 57.99 & 128.26 & 142.99 & 89.76 & & & 4.48 & 249.21 & 524.69 \\
\hline
\end{tabular}


Ottosen et al, 2020. Genet. Biodiv. J, Special issue (Behavioural Instability), 1-60

My

Passive behaviour

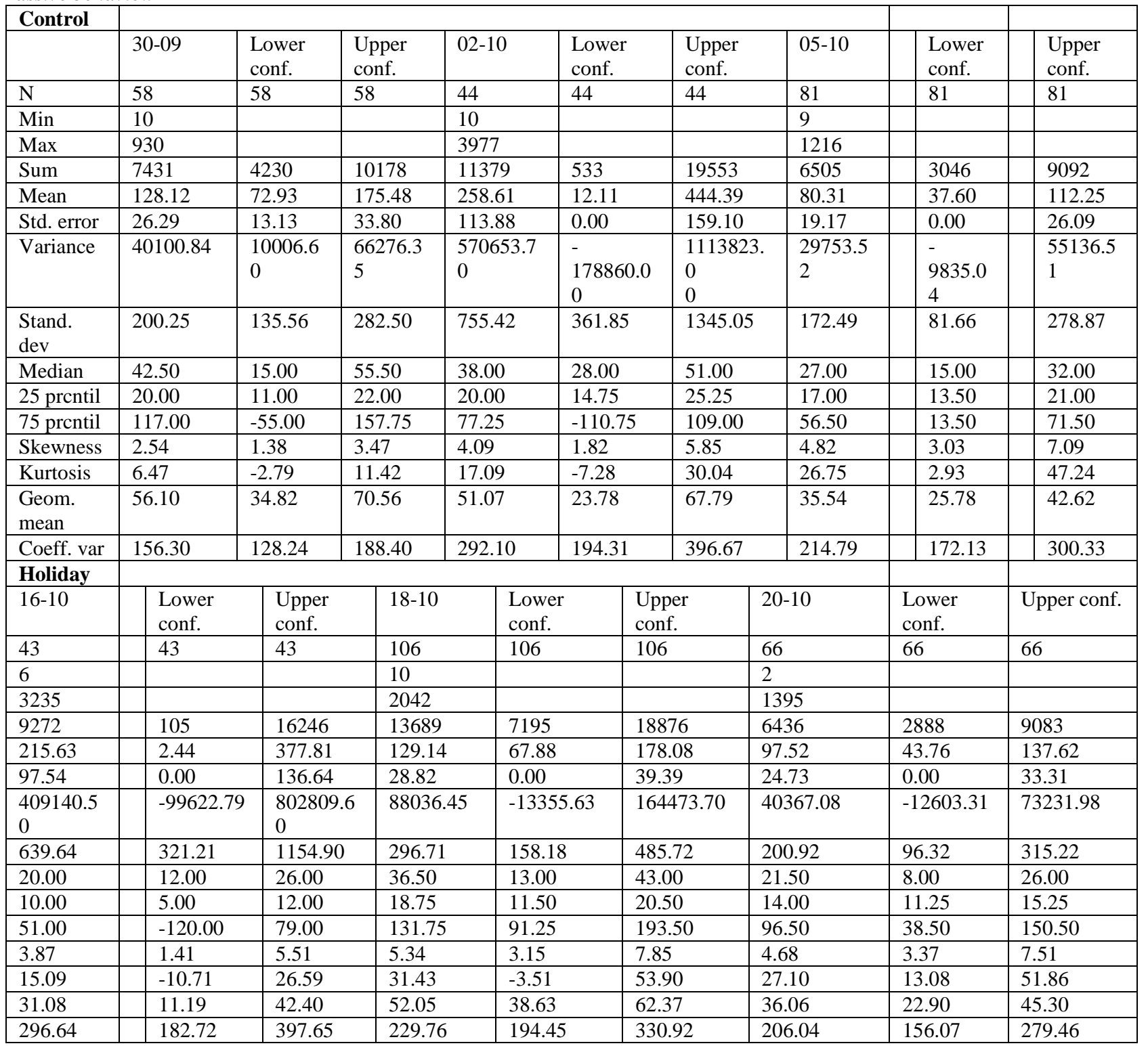


Ottosen et al, 2020. Genet. Biodiv. J, Special issue (Behavioural Instability), 1-60

\begin{tabular}{|c|c|c|c|c|c|c|c|c|c|c|}
\hline \multicolumn{11}{|l|}{ Movement } \\
\hline \multicolumn{11}{|l|}{ Control } \\
\hline & $30-09$ & $\begin{array}{l}\text { Lower } \\
\text { conf. }\end{array}$ & $\begin{array}{l}\text { Upper } \\
\text { conf. }\end{array}$ & \multicolumn{2}{|l|}{$02-10$} & $\begin{array}{l}\text { Lower } \\
\text { conf. }\end{array}$ & $\begin{array}{l}\text { Upper } \\
\text { conf. }\end{array}$ & $05-10$ & $\begin{array}{l}\text { Lower } \\
\text { conf. }\end{array}$ & $\begin{array}{l}\text { Upper } \\
\text { conf. }\end{array}$ \\
\hline $\mathrm{N}$ & 104 & 104 & 104 & 97 & & 97 & 97 & 201 & 201 & 201 \\
\hline Min & 2 & & & 1 & & & & 2 & & \\
\hline Max & 449 & & & 139 & & & & 218 & & \\
\hline Sum & 3974 & 2747 & 4951 & 2438 & & 1904 & 2937 & 4485 & 3774 & 5098 \\
\hline Mean & 38.21 & 26.41 & 47.61 & 25.13 & & 19.63 & 30.28 & 22.31 & 18.78 & 25.36 \\
\hline Std. error & 5.50 & 0.00 & 7.19 & 2.70 & & 1.77 & 3.31 & 1.67 & 0.76 & 2.06 \\
\hline Variance & 3148.11 & -452.87 & 5372.60 & 709.16 & & 305.09 & 1062.38 & 562.70 & 116.34 & 849.76 \\
\hline $\begin{array}{l}\text { Stand. } \\
\text { dev }\end{array}$ & 56.11 & 30.06 & 81.82 & 26.63 & & 19.89 & 34.39 & 23.72 & 15.68 & 30.84 \\
\hline Median & 23.50 & 18.00 & 28.00 & 16.00 & & 11.00 & 20.00 & 14.00 & 10.00 & 16.00 \\
\hline 25 prcntil & 10.00 & 7.00 & 13.00 & 8.00 & & 6.00 & 10.00 & 9.00 & 8.00 & 10.50 \\
\hline 75 prentil & 40.00 & 28.25 & 47.00 & 31.50 & & 20.00 & 38.00 & 29.00 & 25.00 & 34.50 \\
\hline Skewness & 4.59 & 3.32 & 7.29 & 2.19 & & 1.54 & 2.94 & 3.81 & 2.73 & 6.02 \\
\hline Kurtosis & 28.49 & 12.85 & 53.78 & 5.46 & & 0.67 & 9.24 & 23.87 & 11.88 & 45.46 \\
\hline $\begin{array}{l}\text { Geom. } \\
\text { mean }\end{array}$ & 21.10 & 16.20 & 25.14 & 15.69 & & 12.26 & 18.57 & 15.36 & 13.41 & 17.07 \\
\hline $\begin{array}{l}\text { Coeff. } \\
\text { var }\end{array}$ & 146.84 & 110.96 & 195.34 & 105.95 & & 90.45 & 125.02 & 106.31 & 82.07 & 130.32 \\
\hline \multicolumn{11}{|l|}{ Holiday } \\
\hline $16-10$ & $\begin{array}{l}\text { Lower } \\
\text { conf. }\end{array}$ & $\begin{array}{l}\text { Upper } \\
\text { conf. }\end{array}$ & \multicolumn{2}{|l|}{$18-10$} & \multicolumn{2}{|l|}{$\begin{array}{l}\text { Lower } \\
\text { conf. }\end{array}$} & $\begin{array}{l}\text { Upper } \\
\text { conf. }\end{array}$ & $20-10$ & $\begin{array}{l}\text { Lower } \\
\text { conf. }\end{array}$ & Upper conf. \\
\hline 141 & 141 & 141 & 150 & & 150 & & 150 & 159 & 159 & 159 \\
\hline 2 & & & 1 & & & & & 1 & & \\
\hline 213 & & & 259 & & & & & 231 & & \\
\hline 2620 & 2061 & 3062 & 3807 & & 2972 & & 4518 & 3693 & 2855 & 4395 \\
\hline 18.58 & 14.62 & 21.72 & 25.38 & & 19.81 & & 30.12 & 23.23 & 17.96 & 27.64 \\
\hline 1.82 & 0.00 & 2.37 & 2.65 & & 0.95 & & 3.38 & 2.47 & 1.17 & 3.11 \\
\hline 468.45 & -100.47 & 791.26 & 1052.14 & & 135.60 & & 1715.67 & 971.13 & 217.13 & 1535.61 \\
\hline 21.64 & 11.08 & 31.22 & 32.44 & & 20.50 & & 45.16 & 31.16 & 20.79 & 42.16 \\
\hline 13.00 & 12.00 & 16.00 & 16.00 & & 13.00 & & 19.50 & 14.00 & 11.00 & 17.00 \\
\hline 7.50 & 6.00 & 10.00 & 9.00 & & 8.00 & & 11.00 & 7.00 & 6.00 & 9.00 \\
\hline 25.00 & 23.50 & 30.00 & 30.25 & & 27.50 & & 35.25 & 27.00 & 22.00 & 32.00 \\
\hline 5.61 & 4.65 & 10.08 & 4.12 & & 3.00 & & 6.30 & 3.81 & 2.83 & 5.44 \\
\hline 46.44 & 32.84 & 92.24 & 22.76 & & 7.48 & & 41.48 & 18.72 & 6.12 & 32.04 \\
\hline 12.86 & 10.97 & 14.53 & 16.03 & & 13.50 & & 18.22 & 13.58 & 11.17 & 15.56 \\
\hline 116.48 & 78.53 & 159.54 & 127.80 & & 102.61 & & 165.05 & 134.17 & 111.70 & 167.55 \\
\hline
\end{tabular}


Ottosen et al, 2020. Genet. Biodiv. J, Special issue (Behavioural Instability), 1-60

\begin{tabular}{|c|c|c|c|c|c|c|c|c|c|c|c|c|c|c|}
\hline \multicolumn{15}{|l|}{ Foraging } \\
\hline \multirow{2}{*}{\begin{tabular}{|c|} 
Control \\
\end{tabular}} & & \multirow{2}{*}{\multicolumn{2}{|c|}{ Lower conf. }} & \multirow{2}{*}{\multicolumn{2}{|c|}{$\begin{array}{l}\text { Upper } \\
\text { conf. }\end{array}$}} & \multirow{2}{*}{\multicolumn{2}{|c|}{$02-10$}} & \multirow{2}{*}{\multicolumn{2}{|c|}{$\begin{array}{l}\text { Lower } \\
\text { conf. }\end{array}$}} & \multirow{2}{*}{\multicolumn{2}{|c|}{$\begin{array}{l}\text { Upper } \\
\text { conf. }\end{array}$}} & \multirow[b]{2}{*}{$05-10$} & \multirow[b]{2}{*}{$\begin{array}{l}\text { Lower } \\
\text { conf. }\end{array}$} & \multirow[b]{2}{*}{$\begin{array}{l}\text { Upper } \\
\text { conf. }\end{array}$} \\
\hline & $30-09$ & & & & & & & & & & & & & \\
\hline $\mathrm{N}$ & 38 & \multicolumn{2}{|l|}{38} & \multicolumn{2}{|c|}{38} & \multicolumn{2}{|l|}{38} & & & 44 & 44 & 44 \\
\hline Min & 2 & & & & & \multicolumn{2}{|l|}{3} & \multicolumn{2}{|l|}{38} & \multicolumn{2}{|l|}{38} & 2 & & \\
\hline Max & 879 & & & & & \multicolumn{2}{|c|}{606} & & & 741 & & \\
\hline Sum & 3566 & \multicolumn{2}{|l|}{1268} & \multicolumn{2}{|c|}{5377} & \multicolumn{2}{|c|}{5428} & 310 & & 748 & & 4550 & 2173 & 6505 \\
\hline Mean & 93.84 & 33.37 & & 141 & & 142 & & 81.8 & & 197. & & 103.41 & 49.39 & 147.84 \\
\hline Std. error & 28.57 & 0.00 & & 39.2 & & 29. & & 21.0 & & 36.8 & & 26.00 & 10.04 & 35.04 \\
\hline Variance & $\begin{array}{l}31015.0 \\
6\end{array}$ & -4248.15 & & 586 & 4.68 & $\begin{array}{l}33 \\
1\end{array}$ & 7.1 & 168 & 0.78 & $\begin{array}{l}515 \\
4\end{array}$ & 6.1 & $\begin{array}{l}29738.6 \\
2\end{array}$ & $\begin{array}{l}4434.4 \\
7\end{array}$ & $\begin{array}{l}54023.0 \\
0\end{array}$ \\
\hline $\begin{array}{l}\text { Stand. } \\
\text { dev }\end{array}$ & 176.11 & 94.78 & & 293 & & 18 & & 142 & & 241 & & 172.45 & 110.29 & 271.05 \\
\hline Median & 27.50 & 15.50 & & 34.5 & & 52. & & -49 . & & 89.0 & & 41.00 & 17.50 & 56.00 \\
\hline 25 prentil & 17.75 & 13.25 & & 30.5 & & 10. & & -2.7 & & 13.2 & & 21.25 & 15.75 & 33.75 \\
\hline 75 prentil & 94.00 & 2.75 & & 153 & & 200 & & 41.0 & & 280 & & 81.75 & -24.00 & 109.00 \\
\hline $\begin{array}{l}\text { Skewnes } \\
\mathrm{s}\end{array}$ & 3.25 & 1.57 & & 4.8 & & 1.4 & & $0.5 \mathrm{~s}$ & & 2.13 & & 2.77 & 1.01 & 3.87 \\
\hline Kurtosis & 11.43 & -4.37 & & 21.3 & & 1.0 & & -3.3 & & 3.03 & & 7.15 & -9.67 & 12.91 \\
\hline $\begin{array}{l}\text { Geom. } \\
\text { mean }\end{array}$ & 32.10 & 13.37 & & 43.8 & & 48. & & 14.3 & & 68.1 & & 41.10 & 20.49 & 54.77 \\
\hline $\begin{array}{l}\text { Coeff. } \\
\text { var } \\
\end{array}$ & 187.67 & 142.43 & & 256 & & 128 & & 95.9 & & 155 & & 166.76 & 138.19 & 216.22 \\
\hline Holiday & & & & & & & & & & & & & & \\
\hline $16-10$ & & $\begin{array}{l}\text { Lower } \\
\text { conf. }\end{array}$ & $\begin{array}{l}\text { Upl } \\
\text { con }\end{array}$ & & $18-$ & & $\begin{array}{l}\text { Lor } \\
\text { con }\end{array}$ & & $\begin{array}{l}\mathrm{Up} \\
\text { con }\end{array}$ & & $20-$ & & $\begin{array}{l}\text { Lower } \\
\text { conf. }\end{array}$ & Upper conf. \\
\hline 53 & & 53 & 53 & & 25 & & 25 & & 25 & & 41 & & 41 & 41 \\
\hline 2 & & & & & 3 & & & & & & 3 & & & \\
\hline 635 & & & & & 671 & & & & & & 639 & & & \\
\hline 3747 & & 2061 & 514 & & 278 & & 120 & & 404 & & 522 & & 3006 & 7089 \\
\hline 70.70 & & 38.89 & 97. & & 111 & & 48. & & 161 & & 127 & & 73.32 & 172.90 \\
\hline 15.26 & & 0.00 & 19. & & 29.8 & & 0.0 & & 39.8 & & 25. & & 13.13 & 33.52 \\
\hline 12341.29 & & $\begin{array}{l}- \\
237.1 \\
1\end{array}$ & 211 & 3.89 & 222 & 3.14 & -16 & 0.97 & 396 & 0.59 & 270 & 8.84 & 7064.39 & 46060.05 \\
\hline 111.09 & & 64.32 & 162 & & 149 & & 83. & & 228 & & 164 & & 112.01 & 239.43 \\
\hline 22.00 & & -8.00 & 29. & & 51.0 & & 5.0 & & 72. & & 60. & & 6.00 & 83.00 \\
\hline 9.50 & & 4.00 & 12. & & 18.5 & & -6.0 & & 23. & & 30. & & 20.00 & 48.00 \\
\hline 85.50 & & 31.00 & 121 & & 173 & & 64. & & 295 & & 149 & & 62.00 & 197.00 \\
\hline 3.12 & & 2.01 & 4.9 & & 2.52 & & 1.5 & & 4.3 & & 2.1 & & 1.11 & 3.13 \\
\hline 12.44 & & 2.62 & 24.2 & & 7.64 & & 1.6 & & 16. & & 4.3 & & -4.62 & 8.40 \\
\hline 27.26 & & 14.57 & 36. & & 54.1 & & 20. & & 75.2 & & 61. & & 32.18 & 81.82 \\
\hline 157.13 & & $\begin{array}{l}119.0 \\
2\end{array}$ & 202 & & 133 & & 96. & & 178 & & 129 & & 105.75 & 161.26 \\
\hline
\end{tabular}


Ottosen et al, 2020. Genet. Biodiv. $J$, Special issue (Behavioural Instability), 1-60

Reaction to guests/zookeepers
\begin{tabular}{|l|l|l|l|l|l|l|l|l|l|}
\hline Control & & & & & & Holiday & & \\
\hline & $02-10$ & $\begin{array}{l}\text { Lower } \\
\text { conf. }\end{array}$ & $\begin{array}{l}\text { Upper } \\
\text { conf. }\end{array}$ & $05-10$ & $\begin{array}{l}\text { Lower } \\
\text { conf. }\end{array}$ & $\begin{array}{l}\text { Upper } \\
\text { conf. }\end{array}$ & $16-10$ & $\begin{array}{l}\text { Lower } \\
\text { conf. }\end{array}$ & $\begin{array}{l}\text { Upper } \\
\text { conf. }\end{array}$ \\
\hline $\mathrm{N}$ & 6 & 6 & 6 & 7 & 7 & 7 & 20 & 20 & 20 \\
\hline Min & 19 & & & 3 & & & 2 & & \\
\hline Max & 88 & & & 33 & & & 36 & & \\
\hline Sum & 229 & 100 & 317 & 111 & 51 & 166 & 271 & 180 & 354 \\
\hline Mean & 38.17 & 16.67 & 52.83 & 15.86 & 7.29 & 23.71 & 13.55 & 9.00 & 17.70 \\
\hline Std. error & 10.50 & 4.62 & 14.68 & 4.55 & 3.57 & 6.06 & 2.29 & 1.64 & 2.86 \\
\hline Variance & 661.37 & 128.03 & 1292.73 & 144.81 & 89.05 & 257.14 & 105.31 & 53.80 & 163.64 \\
\hline Stand. dev & 25.72 & 16.87 & 45.96 & 12.03 & 9.91 & 18.37 & 10.26 & 8.00 & 13.67 \\
\hline Median & 30.50 & -3.00 & 41.00 & 10.00 & -9.00 & 15.00 & 10.00 & 2.50 & 13.50 \\
\hline 25 prcntil & 20.50 & 6.00 & 22.00 & 5.00 & -12.00 & 7.00 & 6.00 & 3.00 & 10.00 \\
\hline 75 prcntil & 52.00 & 16.00 & 75.75 & 29.00 & 25.00 & 49.00 & 19.50 & 9.00 & 25.00 \\
\hline Skewness & 1.94 & 1.49 & 4.88 & 0.48 & -1.32 & 2.22 & 0.80 & -0.02 & 1.56 \\
\hline Kurtosis & 4.01 & 2.26 & 11.21 & -1.79 & -9.22 & -0.89 & -0.31 & -3.35 & 0.92 \\
\hline Geom. mean & 32.94 & 13.86 & 42.97 & 11.61 & 1.81 & 17.07 & 9.64 & 5.15 & 12.80 \\
\hline Coeff. var & 67.38 & 53.13 & 117.60 & 75.89 & 45.37 & 113.50 & 75.74 & 55.61 & 96.82 \\
\hline
\end{tabular}

\section{Interaction}

\begin{tabular}{|c|c|c|c|c|c|c|c|c|c|}
\hline \multicolumn{10}{|l|}{ Control } \\
\hline & $\begin{array}{l}30-09 \\
\end{array}$ & $\begin{array}{l}\text { Lower } \\
\text { conf. }\end{array}$ & $\begin{array}{l}\text { Upper } \\
\text { conf. }\end{array}$ & $02-10$ & $\begin{array}{l}\text { Lower } \\
\text { conf. }\end{array}$ & $\begin{array}{l}\text { Upper } \\
\text { conf. }\end{array}$ & 05-10 & $\begin{array}{l}\text { Lower } \\
\text { conf. }\end{array}$ & Upper conf. \\
\hline $\mathrm{N}$ & 14 & 14 & 14 & 19 & 19 & 19 & 30 & 30 & 30 \\
\hline Min & 10 & & & 2 & & & 3 & & \\
\hline Max & 98 & & & 78 & & & 499 & & \\
\hline Sum & 461 & 265 & 612 & 424 & 225 & 601 & 1253 & 135 & 1990 \\
\hline Mean & 32.93 & 18.93 & 43.71 & 22.32 & 11.84 & 31.63 & 41.77 & 4.50 & 66.33 \\
\hline Std. error & 6.62 & 1.96 & 9.17 & 5.22 & 3.27 & 6.79 & 16.95 & 0.00 & 23.73 \\
\hline Variance & 612.99 & 53.96 & 1177.77 & 517.67 & 203.06 & 876.21 & 8615.50 & -4879.61 & 16892.25 \\
\hline $\begin{array}{l}\text { Stand. } \\
\text { dev }\end{array}$ & 24.76 & 15.28 & 42.57 & 22.75 & 16.66 & 32.89 & 92.82 & 36.94 & 167.23 \\
\hline Median & 27.00 & 20.50 & 33.00 & 14.00 & 0.00 & 24.00 & 14.50 & 10.00 & 19.50 \\
\hline 25 prentil & 19.00 & 11.00 & 25.75 & 3.00 & -7.00 & 4.00 & 7.00 & 4.00 & 10.00 \\
\hline 75 prentil & 35.00 & -12.25 & 43.00 & 28.00 & -5.00 & 34.00 & 28.00 & -23.00 & 40.00 \\
\hline Skewness & 1.95 & 0.79 & 4.47 & 1.23 & 0.26 & 2.14 & 4.46 & 3.76 & 7.41 \\
\hline Kurtosis & 3.43 & -3.87 & 8.42 & 0.72 & -4.23 & 2.90 & 21.74 & 15.99 & 42.52 \\
\hline $\begin{array}{l}\text { Geom. } \\
\text { mean }\end{array}$ & 26.99 & 16.56 & 34.07 & 12.00 & 3.51 & 17.10 & 16.49 & 7.52 & 22.04 \\
\hline $\begin{array}{l}\text { Coeff. } \\
\text { var }\end{array}$ & 75.19 & 59.98 & 121.62 & 101.96 & 69.68 & 132.52 & 222.23 & 170.51 & 346.67 \\
\hline \multicolumn{10}{|l|}{ Holiday } \\
\hline $16-10$ & $\begin{array}{l}\text { Lower } \\
\text { conf. }\end{array}$ & $\begin{array}{l}\text { Upper } \\
\text { conf. }\end{array}$ & $18-10$ & $\begin{array}{l}\text { Lower } \\
\text { conf. }\end{array}$ & \multicolumn{2}{|c|}{ Upper conf. } & $20-10$ & $\begin{array}{l}\text { Lower } \\
\text { conf. }\end{array}$ & Upper conf. \\
\hline 38 & 38 & 38 & 12 & 12 & \multicolumn{2}{|l|}{12} & 58 & 58 & 58 \\
\hline 1 & & & 1 & & & & 1 & & \\
\hline 193 & & & 512 & & & & 1788 & & \\
\hline 632 & 198 & 899 & 1130 & 50 & \multicolumn{2}{|l|}{1936} & 4806 & 297 & 7928 \\
\hline 16.63 & 5.21 & 23.66 & 94.17 & 4.17 & \multicolumn{2}{|c|}{161.33} & 82.86 & 5.12 & 136.69 \\
\hline 5.00 & 0.00 & 6.99 & 43.64 & 0.00 & \multicolumn{2}{|c|}{60.93} & 34.40 & 0.00 & 47.73 \\
\hline 951.75 & -663.04 & 1854.21 & 22852.52 & -3163.70 & \multirow{2}{*}{\multicolumn{2}{|c|}{$\begin{array}{l}44546.61 \\
268.31 \\
\end{array}$}} & 68651.21 & -35506.12 & 132158.20 \\
\hline 30.85 & 11.04 & 54.68 & 151.17 & 81.28 & & & 262.01 & 108.33 & 452.30 \\
\hline 10.00 & 6.50 & 13.50 & 23.50 & -71.00 & \multicolumn{2}{|c|}{43.00} & 9.00 & 0.00 & 12.00 \\
\hline 4.00 & 0.00 & 5.00 & 3.50 & -19.00 & \multicolumn{2}{|c|}{5.75} & 3.75 & 1.75 & 5.50 \\
\hline 18.00 & 12.50 & 24.00 & 138.50 & -167.00 & \multicolumn{2}{|c|}{249.75} & 26.00 & -8.00 & 33.75 \\
\hline 5.32 & 4.96 & 10.01 & 2.27 & 1.29 & \multicolumn{2}{|c|}{4.04} & 5.36 & 3.96 & 8.25 \\
\hline 30.73 & 27.56 & 61.90 & 5.42 & -0.05 & \multicolumn{2}{|l|}{12.54} & 32.59 & 17.12 & 60.11 \\
\hline 9.16 & 5.64 & 11.68 & 22.47 & -20.78 & \multirow{2}{*}{\multicolumn{2}{|c|}{$\begin{array}{l}37.36 \\
226.39\end{array}$}} & 12.58 & 5.29 & 16.91 \\
\hline 185.49 & 151.39 & 306.19 & 160.54 & 92.98 & & & 316.20 & 224.50 & 435.05 \\
\hline
\end{tabular}


Ottosen et al, 2020. Genet. Biodiv. J, Special issue (Behavioural Instability), 1-60

\section{Appendix G}

1.A Medians for passive behaviour

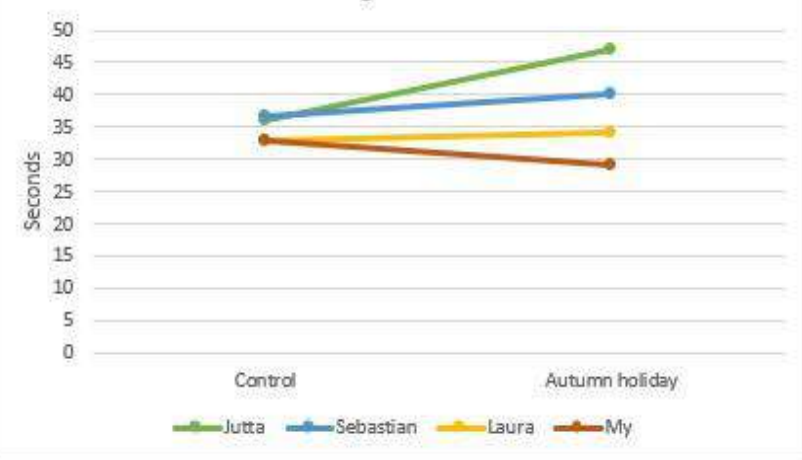

1.C Skewness for passive behaviour

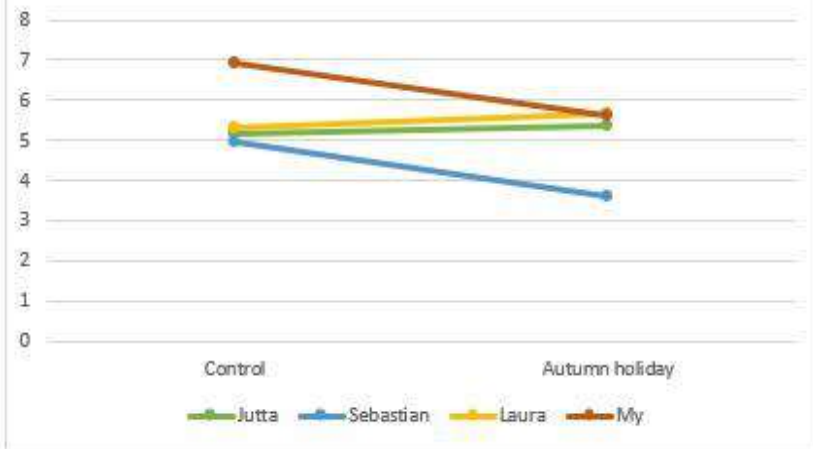

\section{A Medians for movement}
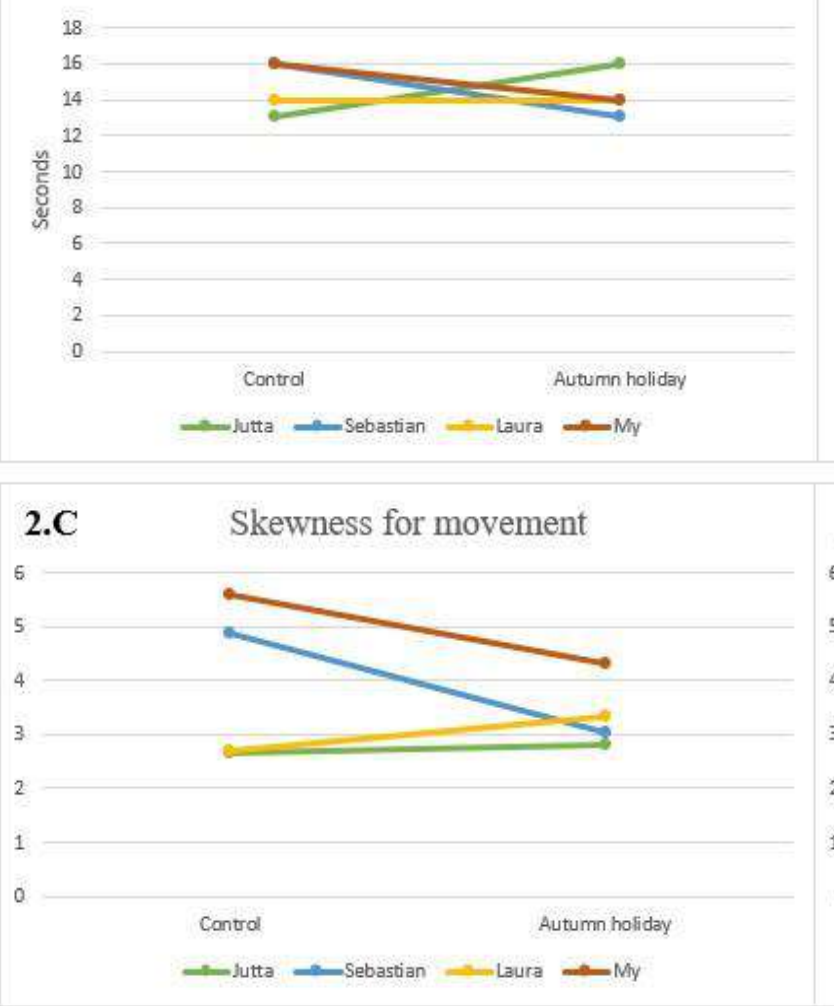

1.B IQR for passive behaviour

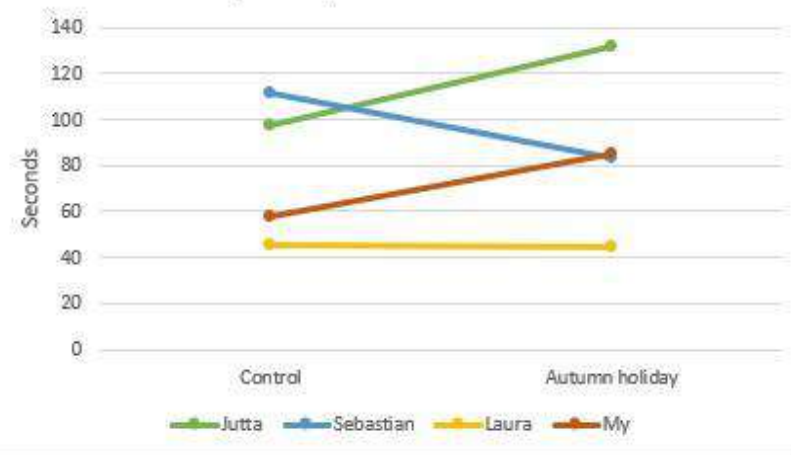

1.D Kurtosis for passive behaviour

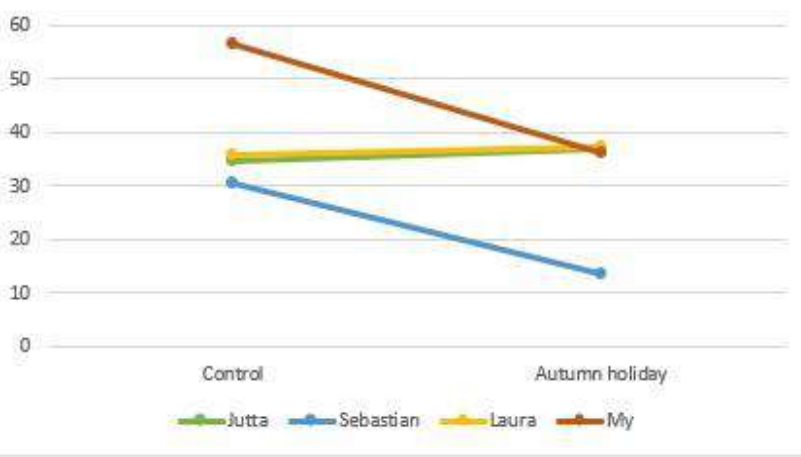

2.B IQR for movement

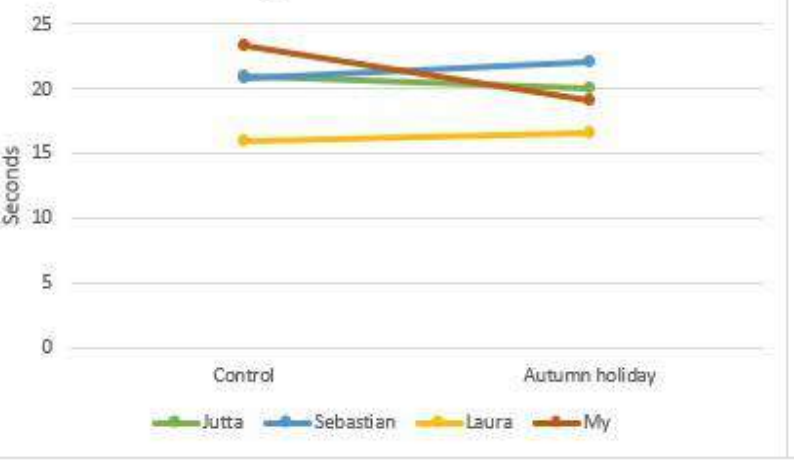

2.D Kurtosis for movement

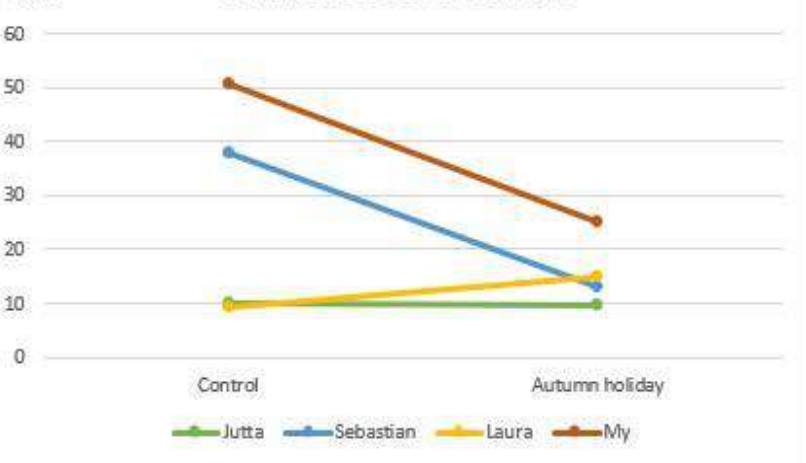


Ottosen et al, 2020. Genet. Biodiv. J, Special issue (Behavioural Instability), 1-60

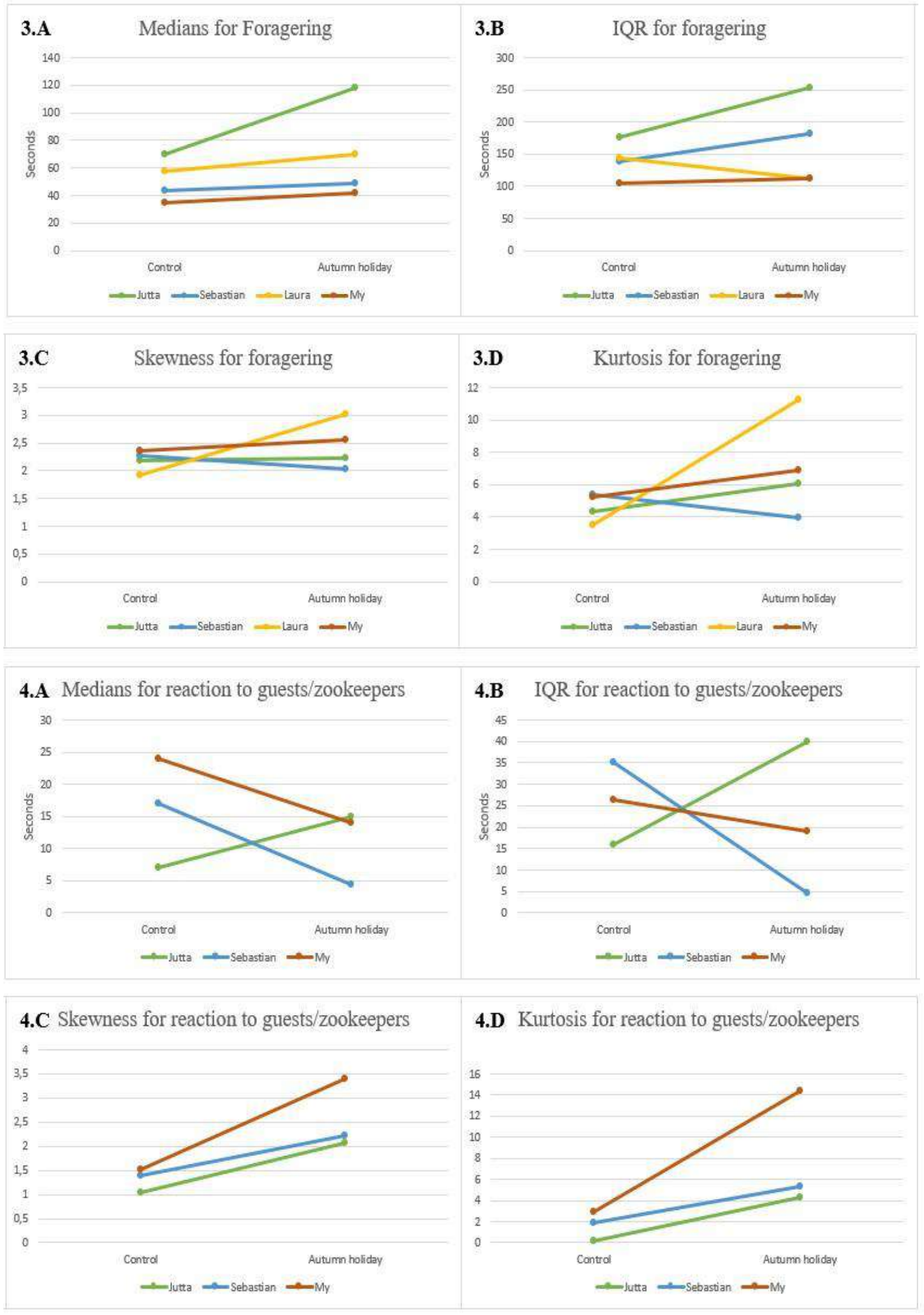


Ottosen et al, 2020. Genet. Biodiv. J, Special issue (Behavioural Instability), 1-60

5.A Medians for stereotype behaviour

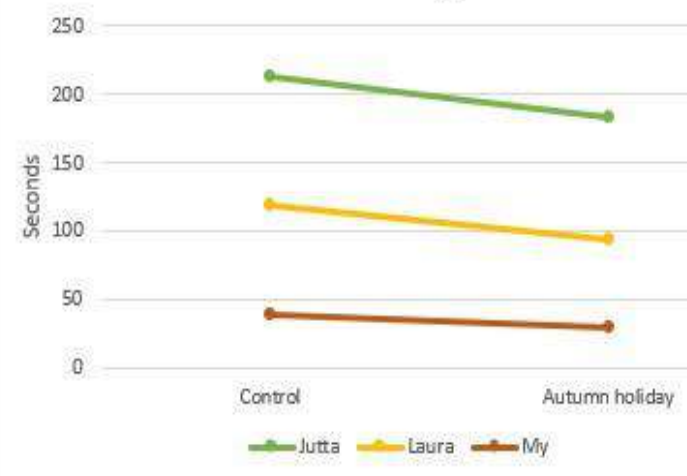

5.C Skewness for stereotype behaviour
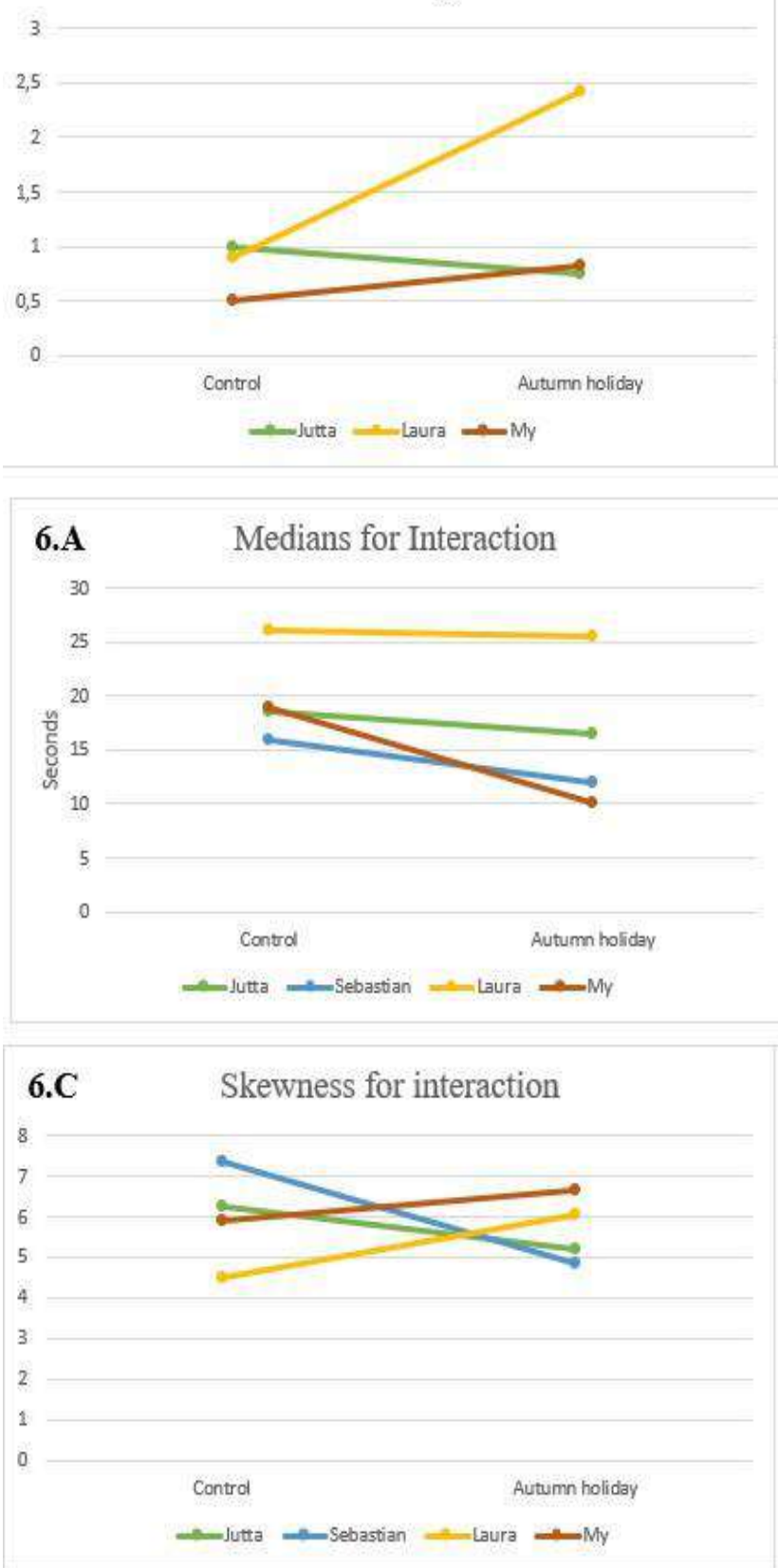

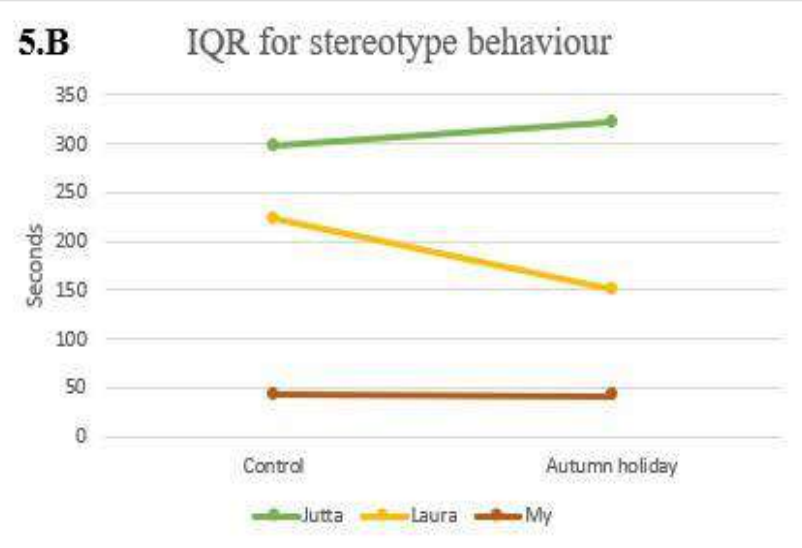

5.D Kurtosis for stereotype behaviour
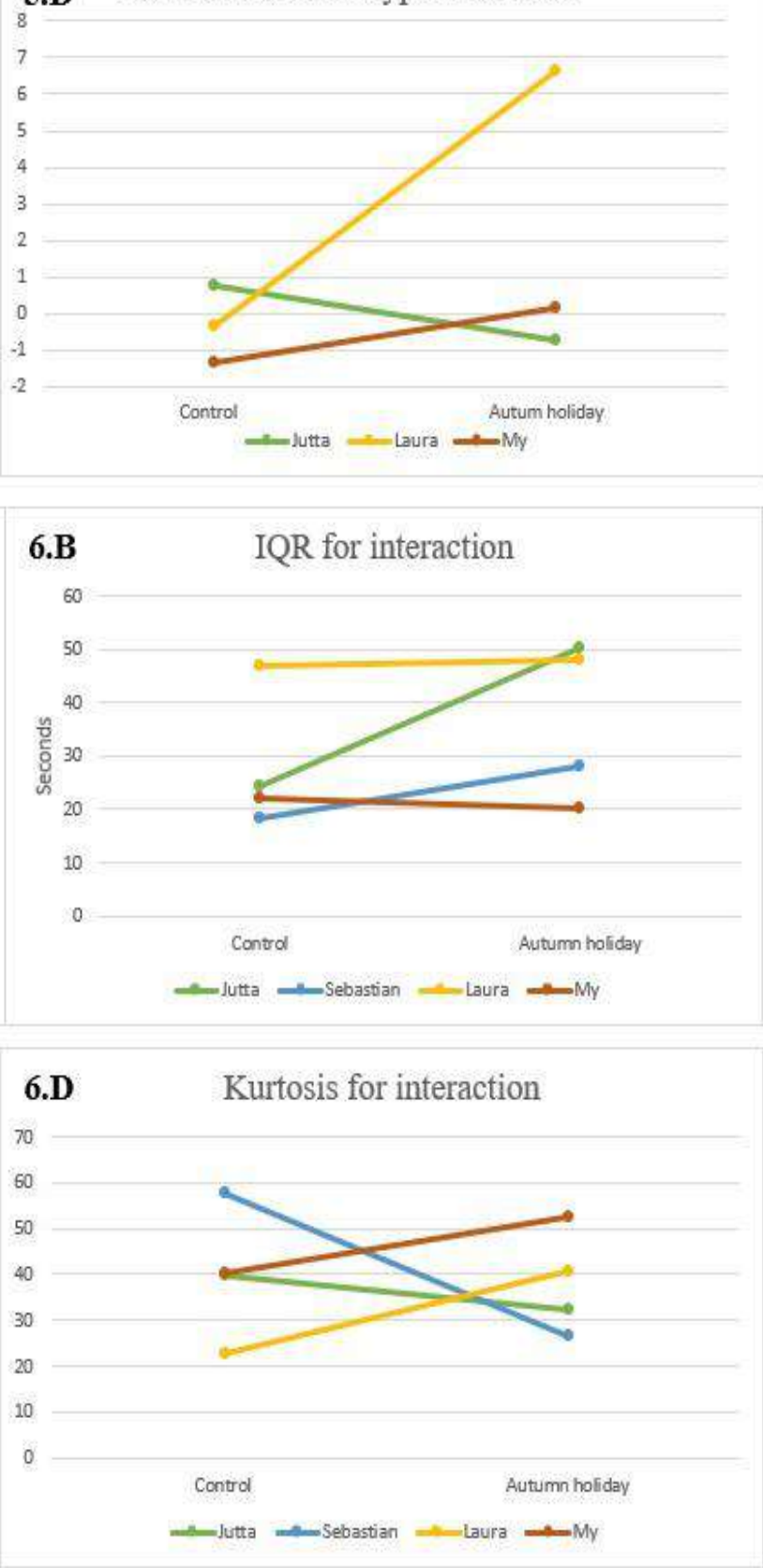


\section{Appendix $\mathbf{H}$}

Comparisons between the chimpanzees Passive behaviour

Kruskal-Wallis test for equal medians (Control)

$\mathrm{H}$ (chi2):

Hc (tie corrected):

There is no significant difference between sample medians

Kruskal-Wallis test for equal medians (Holiday)

$\mathrm{H}$ (chi2):

$\mathrm{Hc}$ (tie corrected):

There is a significant difference between sample medians

\section{Movement}

Kruskal-Wallis test for equal medians (Control)

$\mathrm{H}$ (chi2):

Hc (tie corrected):

$\mathrm{p}$ (same):

There is a significant difference between sample medians

Kruskal-Wallis test for equal medians (Holiday)

$\mathrm{H}$ (chi2):

$\mathrm{Hc}$ (tie corrected):

$\mathrm{p}$ (same):

There is no significant difference between sample medians

Foraging

Kruskal-Wallis test for equal medians (Control)

$\mathrm{H}$ (chi2):

Hc (tie corrected):

$\mathrm{p}$ (same):

There is a significant difference between sample medians

Kruskal-Wallis test for equal medians (Holiday)

$\mathrm{H}$ (chi2):

Hc (tie corrected):

There is a significant difference between sample medians

Reaction to guests/zookeepers

Kruskal-Wallis test for equal medians (Control)

$\mathrm{H}$ (chi2):

Hc (tie corrected):

$\mathrm{p}$ (same):

There is no significant difference between sample medians

Kruskal-Wallis test for equal medians (Holiday)

$\mathrm{H}$ (chi2):

Hc (tie corrected): 
Ottosen et al, 2020. Genet. Biodiv. J, Special issue (Behavioural Instability), 1-60

There is no significant difference between sample medians

Stereotype behaviour

Kruskal-Wallis test for equal medians (Control)

$\mathrm{H}$ (chi2):

Hc (tie corrected):

$\mathrm{p}$ (same):

There is a significant difference between sample medians

Kruskal-Wallis test for equal medians (Holiday)

$\mathrm{H}$ (chi2):

Hc (tie corrected):

11.92

$\mathrm{p}$ (same):

0.002581

There is a significant difference between sample medians

\section{Interaction}

Kruskal-Wallis test for equal medians (Control)

$\mathrm{H}$ (chi2):

Hc (tie corrected): $\quad 6.174$

$\mathrm{p}$ (same): $\quad 0.1034$

There is no significant difference between sample medians

Kruskal-Wallis test for equal medians (Holiday)

$\mathrm{H}$ (chi2):

Hc (tie corrected):

14.84

$\mathrm{p}$ (same):

0.00196

There is a significant difference between sample medians 
Ottosen et al, 2020. Genet. Biodiv. J, Special issue (Behavioural Instability), 1-60

Mann-Whitney pairwise

\begin{tabular}{|c|c|c|c|c|c|c|c|c|c|}
\hline & & Control & & & & Holiday & & & \\
\hline & & Jutta & $\begin{array}{l}\text { Sebasti } \\
\text { an }\end{array}$ & Laura & My & Jutta & $\begin{array}{l}\text { Sebasti } \\
\text { an }\end{array}$ & Laura & My \\
\hline \multirow[t]{4}{*}{ Passive behaviour } & Jutta & & n.s. & n.s. & n.s. & & n.s. & n.s. & $\mathrm{J}>\mathrm{M}^{*}$ \\
\hline & Sebastian & n.s. & & n.s. & n.s. & n.s. & & n.s. & $\mathrm{S}>\mathrm{M}^{*}$ \\
\hline & Laura & n.s. & n.s. & & n.s. & n.s. & n.s. & & n.s. \\
\hline & My & n.s. & n.s. & n.s. & & $\mathrm{J}>\mathrm{M}^{*}$ & $\mathrm{~S}>\mathrm{M}^{*}$ & n.s. & \\
\hline \multirow[t]{4}{*}{ Movement } & Jutta & & n.s. & n.s. & $\mathrm{J}<\mathrm{M}^{* *}$ & & n.s. & n.s. & $\mathrm{J}>\mathrm{M}^{*}$ \\
\hline & Sebastian & n.s. & & n.s. & n.s. & n.s. & & n.s. & n.s. \\
\hline & Laura & n.s. & n.s. & & n.s. & n.s. & n.s. & & n.s. \\
\hline & My & $\mathrm{J}<\mathrm{M}^{* *}$ & n.s. & n.s. & & $\mathrm{J}>\mathrm{M}^{*}$ & n.s. & n.s. & \\
\hline \multirow[t]{4}{*}{ Foraging } & Jutta & & $\mathrm{J}>\mathrm{S}^{*}$ & n.s. & $\mathrm{J}>\mathrm{M}^{* *}$ & & $\mathrm{~J}>\mathrm{S} * *$ & $\mathrm{~J}>\mathrm{L}^{* *}$ & $\mathrm{~J}>\mathrm{M}^{* *}$ \\
\hline & Sebastian & $\mathrm{J}>\mathrm{S}^{*}$ & & n.s. & n.s. & $\mathrm{J}>\mathrm{S} * *$ & & n.s. & n.s. \\
\hline & Laura & n.s. & n.s. & & $\mathrm{L}>\mathrm{M}^{*}$ & $\mathrm{~J}>\mathrm{L} * *$ & n.s. & & n.s. \\
\hline & My & $\mathrm{J}>\mathrm{M}^{* *}$ & n.s. & $\mathrm{L}>\mathrm{M}^{*}$ & & $\mathrm{~J}>\mathrm{M}^{* *}$ & n.s. & n.s. & \\
\hline \multirow{4}{*}{$\begin{array}{l}\text { Reaction to guests/zook } \\
\text { eepers }\end{array}$} & Jutta & & n.s. & n.s. & $\mathrm{J}<\mathrm{M}^{*}$ & & $\mathrm{~J}>\mathrm{S}^{*}$ & - & n.s. \\
\hline & Sebastian & n.s. & & n.s. & n.s. & $\mathrm{J}>\mathrm{S}^{*}$ & & - & n.s. \\
\hline & Laura & n.s. & n.s. & & n.s. & - & - & & - \\
\hline & My & $\mathrm{J}<\mathrm{M}^{*}$ & n.s. & n.s. & & n.s. & n.s. & - & \\
\hline \multirow[t]{4}{*}{ Stereotype behaviour } & Jutta & & - & n.s. & $\mathrm{J}>\mathrm{M}^{* *}$ & & - & n.s. & $\mathrm{J}>\mathrm{M}^{* *}$ \\
\hline & Sebastian & - & & - & - & - & & - & - \\
\hline & Laura & n.s. & - & & $\mathrm{L}>\mathrm{M}^{*}$ & n.s. & - & & $\mathrm{L}>\mathrm{M}^{*}$ \\
\hline & My & $\mathrm{J}>\mathrm{M}^{* *}$ & - & $\mathrm{L}>\mathrm{M}^{*}$ & & $\mathrm{~J}>\mathrm{M}^{* *}$ & - & $\mathrm{L}>\mathrm{M}^{*}$ & \\
\hline \multirow[t]{4}{*}{ Interaction } & Jutta & & n.s. & n.s. & n.s. & & n.s. & n.s. & $\mathrm{J}>\mathrm{M}^{*}$ \\
\hline & Sebastian & n.s. & & $\mathrm{L}>\mathrm{S}^{*}$ & n.s. & n.s. & & $\begin{array}{l}\mathrm{L}>\mathrm{S} * \\
*\end{array}$ & n.s. \\
\hline & Laura & n.s. & $\mathrm{L}>\mathrm{S} *$ & & $\mathrm{~L}>\mathrm{M}^{*}$ & n.s. & $\mathrm{L}>\mathrm{S} * *$ & & $\mathrm{~L}>\mathrm{M}^{* *}$ \\
\hline & My & n.s. & n.s. & $\mathrm{L}>\mathrm{M}^{*}$ & & $\mathrm{~J}>\mathrm{M}^{*}$ & n.s. & $\mathrm{L}>\mathrm{M} * *$ & \\
\hline
\end{tabular}

$* \mathrm{P}<0.05 * * \mathrm{P}<0.01$ 
Ottosen et al, 2020. Genet. Biodiv. J, Special issue (Behavioural Instability), 1-60

IQR

\begin{tabular}{|c|c|c|c|c|c|c|c|c|c|}
\hline & & Control & & & & Holiday & & & \\
\hline & & Jutta & Sebastian & Laura & My & Jutta & Sebastian & Laura & My \\
\hline \multirow{4}{*}{$\begin{array}{l}\text { Passive } \\
\text { behaviour }\end{array}$} & Jutta & & n.s. & $\mathrm{J}>\mathrm{L} * *$ & $\mathrm{~J}>\mathrm{M}^{* *}$ & & $\mathrm{~J}>\mathrm{S} * *$ & $\mathrm{~J}>\mathrm{L} * *$ & $\mathrm{~J}>\mathrm{M}^{* *}$ \\
\hline & Sebastian & n.s. & & $\mathrm{S}>\mathrm{L} * *$ & $\mathrm{~S}>\mathrm{M}^{* *}$ & $\mathrm{~J}>\mathrm{S} * *$ & & $\mathrm{~S}>\mathrm{L} * *$ & n.s. \\
\hline & Laura & $\mathrm{J}>\mathrm{L} * *$ & $\mathrm{~S}>\mathrm{L} * *$ & & n.s. & $\mathrm{J}>\mathrm{L} * *$ & $S>L * *$ & & $\mathrm{M}>\mathrm{L} * *$ \\
\hline & My & $\mathrm{J}>\mathrm{M} * *$ & $\mathrm{~S}>\mathrm{M}^{* *}$ & n.s. & & $\mathrm{J}>\mathrm{M}^{* *}$ & n.s. & $\mathrm{M}>\mathrm{L}^{* *}$ & \\
\hline \multirow[t]{4}{*}{ Movement } & Jutta & & n.s. & $\mathrm{J}>\mathrm{L} *$ & $\mathrm{M}>\mathrm{J} * *$ & & n.s. & $\mathrm{J}>\mathrm{L} * *$ & n.s. \\
\hline & Sebastian & n.s. & & $\mathrm{S}>\mathrm{L} * *$ & $\mathrm{M}>\mathrm{S} * *$ & n.s. & & $\mathrm{S}>\mathrm{L}^{* *}$ & n.s. \\
\hline & Laura & $\mathrm{J}>\mathrm{L} *$ & $\mathrm{~S}>\mathrm{L} * *$ & & $\mathrm{M}>\mathrm{L}^{* *}$ & $\mathrm{~J}>\mathrm{L} * *$ & $\mathrm{~S}>\mathrm{L} * *$ & & $\mathrm{M}>\mathrm{L} * *$ \\
\hline & My & $\mathrm{M}>\mathrm{J} * *$ & $\mathrm{M}>\mathrm{S} * *$ & $\mathrm{M}>\mathrm{L}^{* *}$ & & n.s. & n.s. & $\mathrm{M}>\mathrm{L}^{* *}$ & \\
\hline \multirow[t]{4}{*}{ Foraging } & Jutta & & n.s. & n.s. & $\mathrm{J}>\mathrm{M}^{* *}$ & & n.s. & $\mathrm{J}>\mathrm{L}^{* *}$ & $\mathrm{~J}>\mathrm{M}^{* *}$ \\
\hline & Sebastian & n.s. & & n.s. & $\mathrm{S}>\mathrm{M}^{*}$ & n.s. & & $\mathrm{S}>\mathrm{L} * *$ & $\mathrm{~S}>\mathrm{M}^{* *}$ \\
\hline & Laura & n.s. & n.s. & & $\mathrm{L}>\mathrm{M} * *$ & $\mathrm{~J}>\mathrm{L} * *$ & $\mathrm{~S}>\mathrm{L} * *$ & & n.s. \\
\hline & My & $\mathrm{J}>\mathrm{M}^{* *}$ & $S>M^{*}$ & $\mathrm{~L}>\mathrm{M}^{* *}$ & & $\mathrm{~J}>\mathrm{M}^{* *}$ & $\mathrm{~S}>\mathrm{M}^{* *}$ & n.s. & \\
\hline \multirow{2}{*}{$\begin{array}{l}\text { Reaction } \\
\text { to }\end{array}$} & Jutta & & $\mathrm{S}>\mathrm{J} * *$ & n.s. & n.s. & & $\mathrm{J}>\mathrm{S} *$ & - & n.s. \\
\hline & Sebastian & $\mathrm{S}>\mathrm{J} * *$ & & n.s. & n.s. & $\mathrm{J}>\mathrm{S}^{*}$ & & - & n.s. \\
\hline \multirow{2}{*}{$\begin{array}{l}\text { guests/zoo } \\
\text { keepers }\end{array}$} & Laura & n.s. & n.s. & & n.s. & - & - & & - \\
\hline & My & n.s. & n.s. & n.s. & & n.s. & n.s. & - & \\
\hline \multirow{4}{*}{$\begin{array}{l}\text { Stereotype } \\
\text { behaviour }\end{array}$} & Jutta & & - & n.s. & $\mathrm{J}>\mathrm{M}^{*}$ & & - & n.s. & $\mathrm{J}>\mathrm{M}^{*}$ \\
\hline & Sebastian & - & & - & - & - & & - & - \\
\hline & Laura & n.s. & - & & $\mathrm{L}>\mathrm{M}^{* *}$ & n.s. & - & & n.s. \\
\hline & My & $\mathrm{J}>\mathrm{M}^{*}$ & - & $\mathrm{L}>\mathrm{M}^{* *}$ & & $\mathrm{~J}>\mathrm{M}^{*}$ & - & n.s. & \\
\hline \multirow[t]{4}{*}{ Interaction } & Jutta & & n.s. & n.s. & n.s. & & $\mathrm{J}>\mathrm{S}^{*}$ & n.s. & n.s. \\
\hline & Sebastian & n.s. & & $\mathrm{L}>\mathrm{S} * *$ & n.s. & $\mathrm{J}>\mathrm{S}^{*}$ & & $\mathrm{~L}>\mathrm{S}^{*}$ & n.s. \\
\hline & Laura & n.s. & $\mathrm{L}>\mathrm{S} * *$ & & $\mathrm{~L}>\mathrm{M}^{* *}$ & n.s. & $\mathrm{L}>\mathrm{S} *$ & & n.s. \\
\hline & My & n.s. & n.s. & $\mathrm{L}>\mathrm{M} * *$ & & n.s. & n.s. & n.s. & \\
\hline
\end{tabular}

$* \mathrm{P}<0.05 * * \mathrm{P}<0.01$ 


\section{Appendix I}

Summery statistics for comparisons between the chimpanzees

\section{Passive behaviour}

\begin{tabular}{|c|c|c|c|c|c|c|c|c|c|c|c|c|}
\hline \multicolumn{13}{|l|}{ Control } \\
\hline & Jutta & $\begin{array}{l}\text { Lower } \\
\text { conf. }\end{array}$ & $\begin{array}{l}\text { Upper } \\
\text { conf. }\end{array}$ & $\begin{array}{l}\text { Sebasti } \\
\text { an }\end{array}$ & $\begin{array}{l}\text { Lower } \\
\text { conf. }\end{array}$ & $\begin{array}{l}\text { Upper } \\
\text { conf. }\end{array}$ & Laura & $\begin{array}{l}\text { Lower } \\
\text { conf. }\end{array}$ & $\begin{array}{l}\text { Upper } \\
\text { conf. }\end{array}$ & My & $\begin{array}{l}\text { Lower } \\
\text { conf. }\end{array}$ & $\begin{array}{l}\text { Upper } \\
\text { conf. }\end{array}$ \\
\hline $\mathrm{N}$ & 161 & 161 & 161 & 162 & 162 & 162 & 220 & 220 & 220 & 183 & 183 & 183 \\
\hline Min & 3 & & & 7 & & & 2 & & & 9 & & \\
\hline Max & 3651 & & & 4565 & & & 3601 & & & 3977 & & \\
\hline Sum & $\begin{array}{l}2836 \\
4\end{array}$ & 17157 & 37502 & 35487 & 20885 & 47506 & 32168 & 19825 & 42328 & 25315 & 13138 & 34607 \\
\hline Mean & $\begin{array}{l}176.1 \\
7\end{array}$ & 106.57 & 232.93 & 219.06 & 128.92 & 293.25 & 146.22 & 90.11 & 192.40 & 138.33 & 71.79 & 189.11 \\
\hline $\begin{array}{l}\text { Std. } \\
\text { error }\end{array}$ & 32.60 & 0.00 & 42.41 & 42.81 & 7.89 & 55.53 & 26.14 & 6.77 & 33.48 & 30.07 & 0.00 & 40.66 \\
\hline Variance & $\begin{array}{l}1710 \\
53.50\end{array}$ & $\begin{array}{l}- \\
8664.8 \\
8\end{array}$ & $\begin{array}{l}289573 \\
.60\end{array}$ & $\begin{array}{l}296875 \\
.20\end{array}$ & $\begin{array}{l}10083 . \\
94\end{array}$ & $\begin{array}{l}499532 \\
.10\end{array}$ & $\begin{array}{l}150302 \\
.10\end{array}$ & $\begin{array}{l}10094 . \\
62\end{array}$ & $\begin{array}{l}246577 \\
.00\end{array}$ & $\begin{array}{l}165491 \\
.60\end{array}$ & $\begin{array}{l}- \\
44113 . \\
87\end{array}$ & $\begin{array}{l}302544 \\
.30\end{array}$ \\
\hline $\begin{array}{l}\text { Stand. } \\
\text { dev }\end{array}$ & $\begin{array}{l}413.5 \\
9 \\
\end{array}$ & 234.91 & 597.97 & 544.86 & 325.74 & 782.78 & 387.69 & 236.39 & 542.94 & 406.81 & 201.16 & 644.98 \\
\hline Median & 36.00 & 22.00 & 43.00 & 36.50 & 27.00 & 43.00 & 33.00 & 26.50 & 37.00 & 33.00 & 25.00 & 38.00 \\
\hline $\begin{array}{l}25 \\
\text { prentil }\end{array}$ & 20.00 & 15.50 & 23.00 & 20.00 & 15.00 & 23.00 & 19.00 & 17.00 & 21.00 & 19.00 & 17.00 & 21.00 \\
\hline $\begin{array}{l}75 \\
\text { prentil } \\
\end{array}$ & $\begin{array}{l}1117.5 \\
0 \\
\end{array}$ & 35.00 & 158.00 & 131.25 & 21.00 & 183.50 & 76.75 & 62.00 & 98.50 & 77.00 & 41.00 & 94.00 \\
\hline $\begin{array}{l}\text { Skewnes } \\
\text { s }\end{array}$ & 5.18 & 3.55 & 7.59 & 4.97 & 3.28 & 6.95 & 5.34 & 3.55 & 7.38 & 6.92 & 4.66 & 10.60 \\
\hline Kurtosis & 34.72 & 10.66 & 61.75 & 30.59 & 6.03 & 51.92 & 35.74 & 8.49 & 60.95 & 56.37 & 11.02 & 101.93 \\
\hline $\begin{array}{l}\text { Geom. } \\
\text { mean }\end{array}$ & 54.89 & 41.85 & 64.98 & 58.33 & 43.94 & 69.46 & 44.12 & 35.66 & 50.89 & 44.82 & 35.92 & 51.99 \\
\hline $\begin{array}{l}\text { Coeff. } \\
\text { var }\end{array}$ & $\begin{array}{l}234.7 \\
6\end{array}$ & 186.95 & 296.63 & 248.73 & 201.90 & 308.61 & 265.14 & 217.94 & 319.77 & 294.08 & 241.00 & 407.98 \\
\hline \multicolumn{13}{|l|}{ Holiday } \\
\hline & Jutta & $\begin{array}{l}\text { Lower } \\
\text { conf. }\end{array}$ & $\begin{array}{l}\text { Upper } \\
\text { conf. }\end{array}$ & $\begin{array}{l}\text { Sebasti } \\
\text { an }\end{array}$ & $\begin{array}{l}\text { Lower } \\
\text { conf. }\end{array}$ & $\begin{array}{l}\text { Upper } \\
\text { conf. }\end{array}$ & Laura & $\begin{array}{l}\text { Lower } \\
\text { conf. }\end{array}$ & $\begin{array}{l}\text { Upper } \\
\text { conf. }\end{array}$ & My & $\begin{array}{l}\text { Lower } \\
\text { conf. }\end{array}$ & $\begin{array}{l}\text { Upper } \\
\text { conf. }\end{array}$ \\
\hline $\mathrm{N}$ & 151 & 151 & 151 & 195 & 195 & 195 & 256 & 256 & 256 & 215 & 215 & 215 \\
\hline Min & 3 & & & 3 & & & 2 & & & 2 & & \\
\hline Max & 4342 & & & 2510 & & & 3546 & & & 3235 & & \\
\hline Sum & $\begin{array}{l}3130 \\
6\end{array}$ & 18546 & 41916 & 36123 & 23919 & 47001 & 36810 & 22924 & 48504 & 29397 & 17814 & 38932 \\
\hline Mean & $\begin{array}{l}207.3 \\
2\end{array}$ & 122.82 & 277.59 & 185.25 & 122.66 & 241.03 & 143.79 & 89.55 & 189.47 & 136.73 & 82.86 & 181.08 \\
\hline $\begin{array}{l}\text { Std. } \\
\text { error }\end{array}$ & 39.91 & 0.00 & 52.35 & 29.61 & 18.77 & 36.36 & 25.78 & 10.61 & 33.20 & 25.29 & 7.72 & 32.89 \\
\hline Variance & $\begin{array}{l}2404 \\
69.60\end{array}$ & $\begin{array}{l} \\
31010 . \\
90\end{array}$ & $\begin{array}{l}413879 \\
.50\end{array}$ & $\begin{array}{l}170932 \\
.80\end{array}$ & $\begin{array}{l}68714 . \\
62\end{array}$ & $\begin{array}{l}257734 \\
.70\end{array}$ & $\begin{array}{l}170099 \\
.00\end{array}$ & $\begin{array}{l}28802 . \\
59\end{array}$ & $\begin{array}{l}282222 \\
.30\end{array}$ & $\begin{array}{l}137508 \\
.70\end{array}$ & $\begin{array}{l}12816 . \\
97\end{array}$ & $\begin{array}{l}232552 \\
.50\end{array}$ \\
\hline $\begin{array}{l}\begin{array}{l}\text { Stand. } \\
\text { dev }\end{array} \\
\end{array}$ & $\begin{array}{l}490.3 \\
8 \\
\end{array}$ & 265.25 & 721.80 & 413.44 & 304.24 & 536.83 & 412.43 & 266.83 & 584.08 & 370.82 & 229.59 & 535.57 \\
\hline Median & 47.00 & 24.00 & 61.00 & 40.00 & 30.00 & 46.00 & 34.00 & 30.00 & 38.00 & 29.00 & 23.00 & 35.00 \\
\hline $\begin{array}{l}25 \\
\text { prentil }\end{array}$ & 18.00 & 13.00 & 21.00 & 20.00 & 16.00 & 23.00 & 20.00 & 17.00 & 23.00 & 16.00 & 15.00 & 18.00 \\
\hline $\begin{array}{l}75 \\
\text { prentil }\end{array}$ & $\begin{array}{l}150.0 \\
0\end{array}$ & 56.00 & 181.00 & 103.00 & 26.00 & 134.00 & 64.75 & 52.25 & 74.00 & 101.00 & 63.00 & 133.00 \\
\hline $\begin{array}{l}\text { Skewnes } \\
\text { s }\end{array}$ & 5.34 & 3.72 & 8.06 & 3.58 & 2.44 & 4.40 & 5.67 & 3.92 & 7.33 & 5.63 & 3.53 & 7.21 \\
\hline Kurtosis & 36.91 & 12.27 & 67.49 & 13.46 & 1.77 & 19.82 & 37.09 & 6.30 & 56.87 & 36.04 & 0.24 & 55.03 \\
\hline $\begin{array}{l}\text { Geom. } \\
\text { mean }\end{array}$ & $\begin{array}{l}60.12 \\
\end{array}$ & 44.37 & 72.57 & 52.91 & 40.70 & 62.52 & 43.81 & 36.65 & 49.84 & 41.95 & 33.58 & 48.77 \\
\hline $\begin{array}{l}\text { Coeff. } \\
\text { var }\end{array}$ & $\begin{array}{l}236.5 \\
3\end{array}$ & 185.77 & 301.93 & 223.18 & 194.34 & 252.41 & 286.83 & 246.39 & 352.28 & 271.21 & 231.37 & 334.84 \\
\hline
\end{tabular}


Ottosen et al, 2020. Genet. Biodiv. J, Special issue (Behavioural Instability), 1-60

\begin{tabular}{|c|c|c|c|c|c|c|c|c|c|c|c|c|}
\hline \multicolumn{13}{|c|}{ Movement } \\
\hline Control & & & & & & & & & & & & \\
\hline & Jutta & $\begin{array}{l}\text { Lower } \\
\text { conf. }\end{array}$ & $\begin{array}{l}\text { Upper } \\
\text { conf. }\end{array}$ & $\begin{array}{l}\text { Sebasti } \\
\text { an }\end{array}$ & $\begin{array}{l}\text { Lower } \\
\text { conf. }\end{array}$ & $\begin{array}{l}\text { Upper } \\
\text { conf. }\end{array}$ & Laura & $\begin{array}{l}\text { Lower } \\
\text { conf. }\end{array}$ & $\begin{array}{l}\text { Upper } \\
\text { conf. }\end{array}$ & My & $\begin{array}{l}\text { Lower } \\
\text { conf. }\end{array}$ & $\begin{array}{l}\text { Upper } \\
\text { conf. }\end{array}$ \\
\hline $\mathrm{N}$ & 294 & 294 & 294 & 316 & 316 & 316 & 348 & 348 & 348 & 402 & 402 & 402 \\
\hline Min & 1 & & & 2 & & & 1 & & & 1 & & \\
\hline $\operatorname{Max}$ & 155 & & & 325 & & & 138 & & & 449 & & \\
\hline Sum & 6061 & 5340 & 6737 & 7717 & 6635 & 8696 & 7124 & 6408 & 7819 & 10897 & 9366 & 12188 \\
\hline Mean & 20.62 & 18.16 & 22.91 & 24.42 & 21.00 & 27.52 & 20.47 & 18.41 & 22.47 & 27.11 & 23.30 & 30.32 \\
\hline $\begin{array}{l}\text { Std. } \\
\text { error }\end{array}$ & 1.23 & 0.94 & 1.43 & 1.67 & 0.75 & 2.07 & 1.05 & 0.84 & 1.21 & 1.80 & 0.73 & 2.22 \\
\hline Variance & $\begin{array}{l}443.6 \\
6\end{array}$ & 258.36 & 600.79 & 883.50 & 179.95 & $\begin{array}{l}1353.6 \\
6\end{array}$ & 382.41 & 244.33 & 505.71 & $\begin{array}{l}1303.4 \\
8\end{array}$ & 212.67 & $\begin{array}{l}1981.1 \\
8\end{array}$ \\
\hline $\begin{array}{l}\text { Stand. } \\
\text { dev }\end{array}$ & 21.06 & 17.05 & 25.20 & 29.72 & 19.61 & 39.12 & 19.56 & 16.30 & 23.01 & 36.10 & 23.28 & 47.19 \\
\hline Median & 13.00 & 11.00 & 15.00 & 16.00 & 14.00 & 18.00 & 14.00 & 12.00 & 15.00 & 16.00 & 13.00 & 17.50 \\
\hline $\begin{array}{l}25 \\
\text { prentil }\end{array}$ & 7.00 & 6.00 & 7.00 & 8.00 & 6.00 & 8.75 & 8.00 & 6.00 & 8.00 & 9.00 & 8.00 & 10.00 \\
\hline $\begin{array}{l}75 \\
\text { prentil }\end{array}$ & 28.00 & 23.75 & 34.00 & 28.75 & 24.50 & 32.50 & 24.00 & 20.00 & 25.00 & 32.25 & 27.50 & 35.50 \\
\hline $\begin{array}{l}\text { Skewnes } \\
\text { s }\end{array}$ & 2.64 & 2.00 & 3.60 & 4.87 & 3.60 & 7.72 & 2.69 & 2.17 & 3.28 & 5.58 & 4.16 & 8.70 \\
\hline Kurtosis & 10.06 & 4.68 & 17.12 & 37.69 & 18.42 & 70.45 & 9.20 & 4.59 & 13.39 & 50.81 & 28.01 & 94.13 \\
\hline $\begin{array}{l}\text { Geom. } \\
\text { mean }\end{array}$ & 13.78 & 12.28 & 15.10 & 15.93 & 14.20 & 17.45 & 14.87 & 13.60 & 16.04 & 16.76 & 15.10 & 18.27 \\
\hline $\begin{array}{l}\text { Coeff. } \\
\text { var }\end{array}$ & $\begin{array}{l}102.1 \\
7\end{array}$ & 89.54 & 115.99 & 121.71 & 94.24 & 151.81 & 95.53 & 85.85 & 106.97 & 133.19 & 101.23 & 164.75 \\
\hline \multicolumn{13}{|l|}{ Holiday } \\
\hline & Jutta & $\begin{array}{l}\text { Lower } \\
\text { conf. }\end{array}$ & $\begin{array}{l}\text { Upper } \\
\text { conf. }\end{array}$ & $\begin{array}{l}\text { Sebasti } \\
\text { an }\end{array}$ & $\begin{array}{l}\text { Lower } \\
\text { conf. }\end{array}$ & $\begin{array}{l}\text { Upper } \\
\text { conf. }\end{array}$ & Laura & $\begin{array}{l}\text { Lower } \\
\text { conf. }\end{array}$ & $\begin{array}{l}\text { Upper } \\
\text { conf. }\end{array}$ & My & $\begin{array}{l}\text { Lower } \\
\text { conf. }\end{array}$ & $\begin{array}{l}\text { Upper } \\
\text { conf. }\end{array}$ \\
\hline $\mathrm{N}$ & 255 & 255 & 255 & 392 & 392 & 392 & 366 & 366 & 366 & 450 & 450 & 450 \\
\hline Min & 2 & & & 1 & & & 2 & & & 1 & & \\
\hline Max & 168 & & & 195 & & & 226 & & & 259 & & \\
\hline Sum & 6246 & 5389 & 7037 & 8979 & 7939 & 9930 & 8341 & 7262 & 9295 & 10120 & 8839 & 11240 \\
\hline Mean & 24.49 & 21.13 & 27.60 & 22.91 & 20.25 & 25.33 & 22.79 & 19.84 & 25.40 & 22.49 & 19.64 & 24.98 \\
\hline $\begin{array}{l}\text { Std. } \\
\text { error }\end{array}$ & 1.64 & 1.22 & 1.93 & 1.31 & 1.01 & 1.52 & 1.39 & 1.01 & 1.63 & 1.37 & 0.94 & 1.64 \\
\hline Variance & $\begin{array}{l}684.5 \\
0\end{array}$ & 381.99 & 947.54 & 675.28 & 400.28 & 905.12 & 709.11 & 374.02 & 973.06 & 844.73 & 401.31 & $\begin{array}{l}1206.2 \\
6\end{array}$ \\
\hline $\begin{array}{l}\text { Stand. } \\
\text { dev }\end{array}$ & 26.16 & 20.91 & 31.80 & 25.99 & 21.15 & 30.87 & 26.63 & 20.94 & 32.16 & 29.06 & 22.24 & 36.15 \\
\hline Median & 16.00 & 14.00 & 18.00 & 13.00 & 10.50 & 14.00 & 14.00 & 12.00 & 15.00 & 14.00 & 12.00 & 16.00 \\
\hline $\begin{array}{l}25 \\
\text { prentil }\end{array}$ & 9.00 & 8.00 & 10.00 & 7.00 & 6.00 & 7.00 & 8.75 & 7.50 & 10.50 & 8.00 & 7.00 & 9.25 \\
\hline $\begin{array}{l}75 \\
\text { prentil }\end{array}$ & 29.00 & 24.00 & 32.00 & 29.00 & 25.00 & 32.25 & 25.25 & 22.50 & 28.50 & 27.00 & 24.00 & 30.00 \\
\hline $\begin{array}{l}\text { Skewnes } \\
\text { s }\end{array}$ & 2.82 & 2.29 & 3.44 & 3.02 & 2.47 & 3.95 & 3.35 & 2.54 & 4.26 & 4.32 & 3.62 & 5.43 \\
\hline Kurtosis & 9.76 & 4.94 & 13.80 & 12.96 & 8.00 & 20.15 & 15.04 & 5.92 & 23.40 & 24.99 & 14.56 & 35.34 \\
\hline $\begin{array}{l}\text { Geom. } \\
\text { mean }\end{array}$ & 16.43 & 14.50 & 18.17 & 14.46 & 13.00 & 15.71 & 14.97 & 13.49 & 16.24 & 14.11 & 12.82 & 15.27 \\
\hline $\begin{array}{l}\text { Coeff. } \\
\text { var }\end{array}$ & $\begin{array}{l}106.8 \\
1\end{array}$ & 94.63 & 121.89 & 113.45 & 100.36 & 128.40 & 116.85 & 102.53 & 133.03 & 129.24 & 111.66 & 151.76 \\
\hline
\end{tabular}


Ottosen et al, 2020. Genet. Biodiv. J, Special issue (Behavioural Instability), 1-60

Foraging

\begin{tabular}{|c|c|c|c|c|c|c|c|c|c|c|c|c|}
\hline \multicolumn{13}{|l|}{ Control } \\
\hline & Jutta & $\begin{array}{l}\text { Lower } \\
\text { conf. }\end{array}$ & $\begin{array}{l}\text { Upper } \\
\text { conf. }\end{array}$ & $\begin{array}{l}\text { Sebasti } \\
\text { an }\end{array}$ & $\begin{array}{l}\text { Lower } \\
\text { conf. }\end{array}$ & $\begin{array}{l}\text { Upper } \\
\text { conf. }\end{array}$ & Laura & $\begin{array}{l}\text { Lower } \\
\text { conf. }\end{array}$ & $\begin{array}{l}\text { Upper } \\
\text { conf. }\end{array}$ & My & $\begin{array}{l}\text { Lower } \\
\text { conf. }\end{array}$ & $\begin{array}{l}\text { Upper } \\
\text { conf. }\end{array}$ \\
\hline $\mathrm{N}$ & 96 & 96 & 96 & 111 & 111 & 111 & 90 & 90 & 90 & 120 & 120 & 120 \\
\hline Min & 2 & & & 1 & & & 6 & & & 2 & & \\
\hline Max & 980 & & & 1012 & & & 740 & & & 879 & & \\
\hline Sum & 15456 & 11045 & 19466 & 14882 & 10730 & 18731 & 11532 & 8526 & 14255 & 13544 & 9552 & 17117 \\
\hline Mean & $\begin{array}{l}161.0 \\
0\end{array}$ & 115.05 & 202.77 & 134.07 & 96.67 & 168.75 & 128.13 & 94.73 & 158.39 & 112.87 & 79.60 & 142.64 \\
\hline $\begin{array}{l}\text { Std. } \\
\text { error }\end{array}$ & 22.30 & 15.90 & 27.12 & 18.85 & 13.15 & 22.77 & 16.49 & 11.85 & 19.83 & 16.13 & 11.53 & 19.39 \\
\hline $\begin{array}{l}\text { Varianc } \\
\mathrm{e}\end{array}$ & $\begin{array}{l}47758 \\
.23\end{array}$ & $\begin{array}{l}24284 . \\
53\end{array}$ & $\begin{array}{l}70582 . \\
80\end{array}$ & $\begin{array}{l}39440 . \\
12\end{array}$ & $\begin{array}{l}19188 . \\
18\end{array}$ & $\begin{array}{l}57527 . \\
01\end{array}$ & $\begin{array}{l}24471 . \\
53\end{array}$ & $\begin{array}{l}12636 . \\
04\end{array}$ & $\begin{array}{l}35373 . \\
24\end{array}$ & $\begin{array}{l}31233 . \\
65\end{array}$ & $\begin{array}{l}15962 . \\
05\end{array}$ & $\begin{array}{l}45125 . \\
17\end{array}$ \\
\hline $\begin{array}{l}\text { Stand. } \\
\text { dev }\end{array}$ & $\begin{array}{l}218.5 \\
4 \\
\end{array}$ & 170.18 & 279.17 & 198.60 & 152.87 & 251.06 & 156.43 & 122.32 & 196.38 & 176.73 & 137.81 & 221.77 \\
\hline Median & 69.50 & 36.00 & 88.00 & 44.00 & 25.00 & 56.00 & 58.00 & 25.50 & 73.50 & 34.50 & 21.00 & 43.00 \\
\hline $\begin{array}{l}25 \\
\text { prentil }\end{array}$ & 27.50 & 16.00 & 34.00 & 18.00 & 10.00 & 22.00 & 23.00 & 13.25 & 27.25 & 15.50 & 10.00 & 23.00 \\
\hline $\begin{array}{l}75 \\
\text { prentil }\end{array}$ & $\begin{array}{l}203.2 \\
5\end{array}$ & 148.50 & 273.50 & 157.00 & 51.00 & 219.00 & 167.00 & 66.50 & 210.25 & 120.75 & 56.50 & 166.00 \\
\hline $\begin{array}{l}\text { Skewne } \\
\text { ss }\end{array}$ & 2.19 & 1.43 & 2.80 & 2.27 & 1.50 & 2.98 & 1.92 & 1.28 & 2.54 & 2.37 & 1.57 & 2.98 \\
\hline Kurtosis & 4.31 & -1.17 & 7.24 & 5.35 & 0.07 & 9.28 & 3.52 & -0.39 & 6.34 & 5.24 & -0.60 & 8.51 \\
\hline $\begin{array}{l}\text { Geom. } \\
\text { mean }\end{array}$ & 71.66 & 49.42 & 89.19 & 47.01 & 30.65 & 58.87 & 63.56 & 45.10 & 77.84 & 40.02 & 27.57 & 49.35 \\
\hline $\begin{array}{l}\text { Coeff. } \\
\text { var }\end{array}$ & $\begin{array}{l}135.7 \\
4\end{array}$ & 118.07 & 154.49 & 148.13 & 126.16 & 170.05 & 122.09 & 104.58 & 140.91 & 156.58 & 136.14 & 177.21 \\
\hline \multicolumn{13}{|l|}{ Holiday } \\
\hline & Jutta & $\begin{array}{l}\text { Lower } \\
\text { conf. }\end{array}$ & $\begin{array}{l}\text { Upper } \\
\text { conf. }\end{array}$ & $\begin{array}{l}\text { Sebasti } \\
\text { an }\end{array}$ & $\begin{array}{l}\text { Lower } \\
\text { conf. }\end{array}$ & $\begin{array}{l}\text { Upper } \\
\text { conf. }\end{array}$ & Laura & $\begin{array}{l}\text { Lower } \\
\text { conf. }\end{array}$ & $\begin{array}{l}\text { Upper } \\
\text { conf. }\end{array}$ & My & $\begin{array}{l}\text { Lower } \\
\text { conf. }\end{array}$ & $\begin{array}{l}\text { Upper } \\
\text { conf. }\end{array}$ \\
\hline $\mathrm{N}$ & 72 & 72 & 72 & 93 & 93 & 93 & 83 & 83 & 83 & 119 & 119 & 119 \\
\hline Min & 2 & & & 1 & & & 1 & & & 2 & & \\
\hline Max & 1325 & & & 937 & & & 1166 & & & 671 & & \\
\hline Sum & 15024 & 10765 & 18798 & 13403 & 9367 & 17078 & 10896 & 7108 & 14017 & 11752 & 8607 & 14543 \\
\hline Mean & $\begin{array}{l}208.6 \\
7 \\
\end{array}$ & 149.51 & 261.08 & 144.12 & 100.72 & 183.63 & 131.28 & 85.64 & 168.88 & 98.76 & 72.33 & 122.21 \\
\hline $\begin{array}{l}\text { Std. } \\
\text { error }\end{array}$ & 28.88 & 15.63 & 35.96 & 21.19 & 14.85 & 25.62 & 21.00 & 8.28 & 26.78 & 12.90 & 8.74 & 15.76 \\
\hline $\begin{array}{l}\text { Varianc } \\
\mathrm{e}\end{array}$ & $\begin{array}{l}60038 \\
.90\end{array}$ & $\begin{array}{l}17599 . \\
03\end{array}$ & $\begin{array}{l}93079 . \\
82\end{array}$ & $\begin{array}{l}41775 . \\
41\end{array}$ & $\begin{array}{l}20517 . \\
10\end{array}$ & $\begin{array}{l}61038 . \\
02\end{array}$ & $\begin{array}{l}36596 . \\
25\end{array}$ & $\begin{array}{l}5692.0 \\
9\end{array}$ & $\begin{array}{l}59511 . \\
52\end{array}$ & $\begin{array}{l}19788 . \\
86\end{array}$ & $\begin{array}{l}9099.6 \\
9\end{array}$ & $\begin{array}{l}29548 . \\
11\end{array}$ \\
\hline $\begin{array}{l}\text { Stand. } \\
\text { dev }\end{array}$ & $\begin{array}{l}245.0 \\
3 \\
\end{array}$ & 169.93 & 325.75 & 204.39 & 157.72 & 258.74 & 191.30 & 122.79 & 265.64 & 140.67 & 106.77 & 181.20 \\
\hline Median & $\begin{array}{l}118.5 \\
0\end{array}$ & 72.00 & 160.00 & 49.00 & 10.00 & 69.00 & 70.00 & 55.00 & 97.00 & 42.00 & 26.00 & 54.00 \\
\hline $\begin{array}{l}25 \\
\text { prentil }\end{array}$ & 43.75 & 13.00 & 55.50 & 11.50 & 2.00 & 17.00 & 24.00 & 8.00 & 34.00 & 15.00 & 8.00 & 18.00 \\
\hline $\begin{array}{l}75 \\
\text { prentil }\end{array}$ & $\begin{array}{l}296.2 \\
5 \\
\end{array}$ & 187.50 & 413.50 & 193.50 & 118.00 & 257.50 & 136.00 & 75.00 & 165.00 & 128.00 & 78.00 & 167.00 \\
\hline $\begin{array}{l}\text { Skewne } \\
\text { ss }\end{array}$ & 2.23 & 1.39 & 3.26 & 2.04 & 1.38 & 2.64 & 3.02 & 1.94 & 4.34 & 2.56 & 1.91 & 3.15 \\
\hline Kurtosis & 6.08 & \begin{tabular}{|c|}
-0.19 \\
\end{tabular} & 11.51 & 3.95 & -0.46 & 6.78 & 11.27 & 1.51 & 20.47 & $\begin{array}{l}6.91 \\
\end{array}$ & 1.50 & 10.53 \\
\hline $\begin{array}{l}\text { Geom. } \\
\text { mean }\end{array}$ & $\begin{array}{l}102.3 \\
6 \\
\end{array}$ & 64.21 & 130.75 & 44.51 & 25.25 & 58.21 & 57.22 & 36.74 & 72.33 & 41.68 & 30.06 & 51.13 \\
\hline $\begin{array}{l}\text { Coeff. } \\
\text { var }\end{array}$ & $\begin{array}{l}117.4 \\
3\end{array}$ & 95.40 & 141.63 & 141.82 & 119.65 & 163.77 & 145.72 & 118.49 & 178.74 & 142.44 & 123.58 & 164.77 \\
\hline
\end{tabular}


Ottosen et al, 2020. Genet. Biodiv. J, Special issue (Behavioural Instability), 1-60

\begin{tabular}{|c|c|c|c|c|c|c|c|c|c|c|c|c|}
\hline \multirow{2}{*}{\multicolumn{13}{|c|}{ Reaction to guests/zookeepers }} \\
\hline Control & & \multirow[b]{2}{*}{$\begin{array}{l}\text { Lower } \\
\text { conf. }\end{array}$} & \multirow[b]{2}{*}{$\begin{array}{l}\text { Upper } \\
\text { conf. }\end{array}$} & \multirow[b]{2}{*}{$\begin{array}{l}\text { Seb. } \\
\text { (Reak.) } \\
\text { C }\end{array}$} & \multirow[b]{2}{*}{$\begin{array}{l}\text { Lower } \\
\text { conf. }\end{array}$} & \multirow[b]{2}{*}{$\begin{array}{l}\text { Upper } \\
\text { conf. }\end{array}$} & \multirow[b]{2}{*}{$\begin{array}{l}\text { Laura } \\
\text { (Reak.) } \\
\text { C }\end{array}$} & \multirow[b]{2}{*}{$\begin{array}{l}\text { Lower } \\
\text { conf. }\end{array}$} & \multirow[b]{2}{*}{$\begin{array}{l}\text { Upper } \\
\text { conf. }\end{array}$} & \multirow[b]{2}{*}{$\begin{array}{l}\text { My } \\
\text { (Reak. } \\
\text { ) C }\end{array}$} & \multirow[b]{2}{*}{$\begin{array}{l}\text { Lower } \\
\text { conf. }\end{array}$} & \multirow[b]{2}{*}{$\begin{array}{l}\text { Upper } \\
\text { conf. }\end{array}$} \\
\hline & $\begin{array}{l}\text { Jutta } \\
\text { (Reak.) } \\
\text { C }\end{array}$ & & & & & & & & & & & \\
\hline $\mathrm{N}$ & 15 & 15 & 15 & 8 & 8 & 8 & 7 & 7 & 7 & 14 & 14 & 14 \\
\hline Min & 2 & & & 2 & & & 5 & & & 3 & & \\
\hline Max & 33 & & & 86 & & & 48 & & & 88 & & \\
\hline Sum & 173 & 95 & 240 & 213 & 49 & 346 & 157 & 71 & 235 & 396 & 222 & 538 \\
\hline Mean & 11.53 & 6.33 & 16.00 & 26.63 & 6.13 & 43.25 & 22.43 & 10.14 & 33.57 & 28.29 & 15.86 & 38.43 \\
\hline $\begin{array}{l}\text { Std. } \\
\text { error }\end{array}$ & 2.49 & 1.62 & 3.24 & 10.15 & 4.69 & 13.74 & 6.43 & 4.74 & 8.70 & 6.03 & 1.38 & 8.06 \\
\hline $\begin{array}{l}\text { Varian } \\
\text { ce }\end{array}$ & 92.98 & 39.56 & 157.61 & 824.27 & 175.84 & $\begin{array}{l}1510.5 \\
4\end{array}$ & 289.62 & 157.33 & 530.33 & $\begin{array}{l}508.5 \\
3\end{array}$ & 26.83 & 910.50 \\
\hline $\begin{array}{l}\text { Stand. } \\
\text { dev }\end{array}$ & 9.64 & 7.19 & 13.96 & 28.71 & 19.04 & 45.67 & 17.02 & 13.50 & 27.04 & 22.55 & 13.63 & 34.78 \\
\hline Median & 7.00 & -5.00 & 8.00 & 17.00 & -7.00 & 29.00 & 21.00 & 0.00 & 35.00 & 24.00 & 13.00 & 33.50 \\
\hline $\begin{array}{l}25 \\
\text { prentil }\end{array}$ & 3.00 & -1.00 & 4.00 & 5.25 & -16.50 & 8.50 & 7.00 & -10.00 & 9.00 & 9.75 & -4.75 & 15.00 \\
\hline $\begin{array}{l}75 \\
\text { prentil }\end{array}$ & 19.00 & 11.00 & 31.00 & 40.50 & -5.00 & 74.00 & 42.00 & 36.00 & 74.00 & 36.25 & 8.50 & 46.50 \\
\hline $\begin{array}{l}\text { Skewn } \\
\text { ess }\end{array}$ & 1.05 & -0.04 & 2.05 & 1.39 & 0.22 & 3.54 & 0.63 & -0.84 & 2.41 & 1.51 & 0.64 & 3.43 \\
\hline $\begin{array}{l}\text { Kurtosi } \\
\text { s }\end{array}$ & 0.18 & -4.59 & 2.03 & 1.87 & -3.26 & 6.21 & -1.24 & -7.22 & 0.19 & 2.92 & -1.34 & 7.38 \\
\hline $\begin{array}{l}\text { Geom. } \\
\text { mean }\end{array}$ & 8.06 & 3.37 & 11.00 & 14.10 & -3.92 & 22.10 & 16.66 & 3.23 & 24.22 & 20.31 & 8.75 & 27.95 \\
\hline $\begin{array}{l}\text { Coeff. } \\
\text { var }\end{array}$ & 83.61 & 60.92 & 110.21 & 107.83 & 58.43 & 160.70 & 75.88 & 45.90 & 113.06 & 79.72 & 55.05 & 115.38 \\
\hline \multicolumn{3}{|l|}{ Holiday } & & & & & & & & & & \\
\hline & Jutta & $\begin{array}{l}\text { Lower } \\
\text { conf. }\end{array}$ & $\begin{array}{l}\text { Upper } \\
\text { conf. }\end{array}$ & $\begin{array}{l}\text { Sebasti } \\
\text { an }\end{array}$ & $\begin{array}{l}\text { Lower } \\
\text { conf. }\end{array}$ & $\begin{array}{l}\text { Upper } \\
\text { conf. }\end{array}$ & My & $\begin{array}{l}\text { Lower } \\
\text { conf. }\end{array}$ & $\begin{array}{l}\text { Upper } \\
\text { conf. }\end{array}$ & & & \\
\hline $\mathrm{N}$ & 7 & 7 & 7 & 8 & 8 & 8 & 27 & 27 & 27 & & & \\
\hline Min & 8 & & & 2 & & & 1 & & & & & \\
\hline Max & 116 & & & 23 & & & 114 & & & & & \\
\hline Sum & 227 & 18 & 374 & 59 & 19 & 86 & 493 & 243 & 678 & & & \\
\hline Mean & 32.43 & 2.57 & 53.43 & 7.38 & 2.38 & 10.75 & 18.26 & 9.00 & 25.11 & & & \\
\hline $\begin{array}{l}\text { Std. } \\
\text { error }\end{array}$ & 14.88 & 4.70 & 20.98 & 2.38 & 0.00 & 3.32 & 4.22 & 0.00 & 5.72 & & & \\
\hline $\begin{array}{l}\text { Varian } \\
\text { ce }\end{array}$ & $\begin{array}{l}1549.2 \\
9\end{array}$ & 154.57 & $\begin{array}{l}3082.5 \\
7\end{array}$ & 45.13 & -2.82 & 88.32 & 479.97 & $\begin{array}{l}- \\
197.63\end{array}$ & 881.91 & & & \\
\hline $\begin{array}{l}\text { Stand. } \\
\text { dev }\end{array}$ & 39.36 & 24.46 & 74.72 & 6.72 & 3.79 & 12.05 & 21.91 & 9.79 & 34.98 & & & \\
\hline Median & 15.00 & $\begin{array}{l}-18.00 \\
\end{array}$ & 22.00 & 4.50 & 0.00 & 5.00 & 14.00 & 8.00 & 21.00 & & & \\
\hline $\begin{array}{l}25 \\
\text { prcntil }\end{array}$ & 8.00 & -4.00 & 8.00 & 4.00 & 3.00 & 6.00 & 6.00 & 3.00 & 10.00 & & & \\
\hline $\begin{array}{l}75 \\
\text { prentil }\end{array}$ & 48.00 & -20.00 & 84.00 & 8.75 & -5.50 & 13.50 & 25.00 & 20.00 & 35.00 & & & \\
\hline $\begin{array}{l}\text { Skewn } \\
\text { ess }\end{array}$ & 2.08 & 1.53 & 4.51 & 2.22 & 1.72 & 5.11 & 3.40 & 2.87 & 6.69 & & & \\
\hline $\begin{array}{l}\text { Kurtosi } \\
\text { s }\end{array}$ & 4.29 & 1.68 & 11.36 & 5.35 & 3.13 & 13.13 & 14.44 & 10.92 & 30.35 & & & \\
\hline $\begin{array}{l}\text { Geom. } \\
\text { mean }\end{array}$ & 20.01 & -1.21 & 29.16 & 5.66 & 1.89 & 7.66 & 10.92 & 5.58 & 14.54 & & & \\
\hline $\begin{array}{l}\text { Coeff. } \\
\text { var }\end{array}$ & 121.38 & 89.63 & 212.46 & 91.08 & 69.25 & 151.76 & 119.98 & 88.39 & 179.35 & & & \\
\hline
\end{tabular}


Ottosen et al, 2020. Genet. Biodiv. J, Special issue (Behavioural Instability), 1-60

Stereotype behaviour

\begin{tabular}{|c|c|c|c|c|c|c|c|c|c|}
\hline \multicolumn{2}{|l|}{ Control } & \multirow[b]{2}{*}{$\begin{array}{l}\text { Lower } \\
\text { conf. }\end{array}$} & \multirow[b]{2}{*}{$\begin{array}{l}\text { Upper } \\
\text { conf. }\end{array}$} & \multirow[b]{2}{*}{ Laura } & \multirow[b]{2}{*}{$\begin{array}{l}\text { Lower } \\
\text { conf. }\end{array}$} & \multirow[b]{2}{*}{$\begin{array}{l}\text { Upper } \\
\text { conf. }\end{array}$} & \multirow[b]{2}{*}{ My } & \multirow[b]{2}{*}{$\begin{array}{l}\text { Lower } \\
\text { conf. }\end{array}$} & \multirow[b]{2}{*}{$\begin{array}{l}\text { Upper } \\
\text { conf. }\end{array}$} \\
\hline & Jutta & & & & & & & & \\
\hline $\mathrm{N}$ & 9 & 9 & 9 & 13 & 13 & 13 & 9 & 9 & 9 \\
\hline Min & 12 & & & 13 & & & 26 & & \\
\hline Max & 696 & & & 415 & & & 85 & & \\
\hline Sum & 2489 & 1278 & 3563 & 1987 & 1065 & 2806 & 436 & 313 & 553 \\
\hline Mean & 276.56 & 142.00 & 395.89 & 152.85 & 81.92 & 215.85 & 48.44 & 34.78 & 61.44 \\
\hline Std. error & 69.63 & 34.57 & 94.20 & 35.58 & 24.54 & 46.30 & 7.44 & 5.56 & 9.65 \\
\hline Variance & 43639.53 & 10754.53 & 79862.19 & 16453.97 & 7830.64 & 27867.36 & 497.78 & 278.28 & 837.61 \\
\hline Stand. dev & 208.90 & 141.17 & 331.68 & 128.27 & 98.19 & 185.55 & 22.31 & 17.84 & 32.05 \\
\hline Median & 213.00 & -23.00 & 294.00 & 118.00 & -30.00 & 183.00 & 39.00 & 6.00 & 52.00 \\
\hline 25 prentil & 135.00 & 54.50 & 258.00 & 47.00 & -17.00 & 70.50 & 26.50 & 6.50 & 27.00 \\
\hline 75 prentil & 433.50 & 171.00 & 654.00 & 271.00 & 169.00 & 424.00 & 70.00 & 55.00 & 101.00 \\
\hline Skewness & 1.00 & -0.22 & 2.62 & 0.90 & -0.32 & 1.99 & 0.51 & -0.99 & 1.82 \\
\hline Kurtosis & 0.78 & -4.37 & 3.71 & -0.34 & -5.72 & 1.19 & -1.35 & -7.37 & -0.39 \\
\hline $\begin{array}{l}\text { Geom. } \\
\text { mean }\end{array}$ & 186.79 & 27.60 & 288.53 & 102.48 & 33.59 & 145.43 & 44.01 & 29.64 & 54.72 \\
\hline Coeff. var & 75.54 & 45.65 & 110.08 & 83.92 & 57.20 & 111.97 & 46.05 & 35.94 & 64.36 \\
\hline \multicolumn{10}{|l|}{ Holiday } \\
\hline & Jutta & $\begin{array}{l}\text { Lower } \\
\text { conf. }\end{array}$ & $\begin{array}{l}\text { Upper } \\
\text { conf. }\end{array}$ & Laura & $\begin{array}{l}\text { Lower } \\
\text { conf. }\end{array}$ & $\begin{array}{l}\text { Upper } \\
\text { conf. }\end{array}$ & My & $\begin{array}{l}\text { Lower } \\
\text { conf. }\end{array}$ & $\begin{array}{l}\text { Upper } \\
\text { conf. }\end{array}$ \\
\hline $\mathrm{N}$ & 12 & 12 & 12 & 12 & 12 & 12 & 7 & 7 & 7 \\
\hline Min & 40 & & & 11 & & & 10 & & \\
\hline Max & 587 & & & 626 & & & 79 & & \\
\hline Sum & 2840 & 1612 & 3950 & 1723 & 445 & 2611 & 258 & 131 & 369 \\
\hline Mean & 236.67 & 134.33 & 329.17 & 143.58 & 37.08 & 217.58 & 36.86 & 18.71 & 52.71 \\
\hline Std. error & 51.83 & 36.06 & 66.67 & 48.77 & 0.00 & 67.77 & 9.18 & 5.23 & 12.48 \\
\hline Variance & 32234.24 & 15608.15 & 53344.79 & 28537.36 & -7397.92 & 55118.33 & 589.81 & 191.71 & 1089.81 \\
\hline Stand. dev & 179.54 & 138.03 & 253.61 & 168.93 & 83.95 & 293.63 & 24.29 & 17.14 & 39.10 \\
\hline Median & 183.50 & -39.00 & 276.50 & 94.00 & 19.50 & 142.50 & 30.00 & 3.00 & 46.00 \\
\hline 25 prentil & 89.75 & 18.50 & 131.00 & 39.25 & -15.25 & 66.25 & 14.00 & -11.00 & 18.00 \\
\hline 75 prentil & 411.00 & 272.50 & 620.00 & 189.75 & -154.75 & 282.50 & 57.00 & 35.00 & 85.00 \\
\hline Skewness & 0.75 & -0.53 & 1.89 & 2.43 & 1.73 & 4.95 & 0.83 & -0.37 & 2.62 \\
\hline Kurtosis & -0.72 & -5.51 & 0.67 & 6.64 & 2.96 & 14.98 & 0.12 & -4.40 & 2.85 \\
\hline $\begin{array}{l}\text { Geom. } \\
\text { mean }\end{array}$ & 174.90 & 73.06 & 240.04 & 83.75 & 11.11 & 122.40 & 29.91 & 10.71 & 41.71 \\
\hline Coeff. var & 75.86 & 53.49 & 102.44 & 117.65 & 83.52 & 182.61 & 65.89 & 41.78 & 101.73 \\
\hline
\end{tabular}


Ottosen et al, 2020. Genet. Biodiv. J, Special issue (Behavioural Instability), 1-60

Interaction

\begin{tabular}{|c|c|c|c|c|c|c|c|c|c|c|c|c|}
\hline \multicolumn{13}{|l|}{ Control } \\
\hline & Jutta & $\begin{array}{l}\text { Lower } \\
\text { conf. }\end{array}$ & $\begin{array}{l}\text { Upper } \\
\text { conf. }\end{array}$ & $\begin{array}{l}\text { Sebasti } \\
\text { an }\end{array}$ & $\begin{array}{l}\text { Lower } \\
\text { conf. }\end{array}$ & $\begin{array}{l}\text { Upper } \\
\text { conf. }\end{array}$ & Laura & $\begin{array}{l}\text { Lower } \\
\text { conf. }\end{array}$ & $\begin{array}{l}\text { Upper } \\
\text { conf. }\end{array}$ & My & $\begin{array}{l}\text { Lower } \\
\text { conf. }\end{array}$ & $\begin{array}{l}\text { Upper } \\
\text { conf. }\end{array}$ \\
\hline $\mathrm{N}$ & 42 & 42 & 42 & 70 & 70 & 70 & 39 & 39 & 39 & 63 & 63 & 63 \\
\hline Min & 2 & & & 3 & & & 3 & & & 2 & & \\
\hline Max & 1114 & & & 626 & & & 1114 & & & 499 & & \\
\hline Sum & 2170 & -150 & 3502 & 2183 & 769 & 3045 & 3223 & 456 & 5077 & 2138 & 932 & 2935 \\
\hline Mean & 51.67 & -3.57 & 83.38 & 31.19 & 10.99 & 43.50 & 82.64 & 11.69 & 130.18 & 33.94 & 14.79 & 46.59 \\
\hline $\begin{array}{l}\text { Std. } \\
\text { error }\end{array}$ & 26.22 & 0.00 & 36.98 & 9.02 & 0.00 & 12.63 & 30.95 & 0.00 & 43.38 & 8.34 & 0.00 & 11.48 \\
\hline $\begin{array}{l}\text { Varian } \\
\text { ce }\end{array}$ & $\begin{array}{l}28869 . \\
15\end{array}$ & $\begin{array}{l}- \\
23740 . \\
06\end{array}$ & $\begin{array}{l}57450 . \\
72\end{array}$ & $\begin{array}{l}5695.3 \\
7\end{array}$ & $\begin{array}{l}- \\
4355.0 \\
1\end{array}$ & $\begin{array}{l}11161 . \\
73\end{array}$ & $\begin{array}{l}37369 \\
13\end{array}$ & $\begin{array}{l}- \\
19629 . \\
12\end{array}$ & $\begin{array}{l}73387 . \\
74\end{array}$ & $\begin{array}{l}4379.9 \\
3\end{array}$ & $\begin{array}{l}- \\
2535.0 \\
5\end{array}$ & $\begin{array}{l}8306.8 \\
0\end{array}$ \\
\hline $\begin{array}{l}\text { Stand. } \\
\text { dev }\end{array}$ & 169.91 & 54.37 & 322.86 & 75.47 & 25.45 & 135.80 & 193.31 & 79.43 & 349.87 & 66.18 & 26.08 & 111.08 \\
\hline Median & 18.50 & 13.00 & 25.00 & 16.00 & 12.00 & 20.00 & 26.00 & 8.00 & 33.00 & 19.00 & 12.00 & 25.00 \\
\hline $\begin{array}{l}25 \\
\text { prentil }\end{array}$ & 8.75 & 3.25 & 12.50 & 9.00 & 6.00 & 11.00 & 11.00 & 0.00 & 13.00 & 7.00 & 1.00 & 10.00 \\
\hline $\begin{array}{l}75 \\
\text { prentil }\end{array}$ & 33.25 & 13.50 & 44.50 & 27.25 & 18.25 & 33.50 & 58.00 & 17.00 & 79.00 & 29.00 & -1.00 & 32.00 \\
\hline $\begin{array}{l}\text { Skewn } \\
\text { ess }\end{array}$ & 6.25 & 6.12 & 11.30 & 7.34 & 6.83 & 13.14 & 4.52 & 3.18 & 7.04 & 5.89 & 5.07 & 10.29 \\
\hline $\begin{array}{l}\text { Kurtosi } \\
\text { s }\end{array}$ & 39.92 & 38.70 & 78.99 & 57.94 & 51.77 & 113.58 & 22.47 & 9.45 & 41.73 & 40.41 & 31.30 & 79.14 \\
\hline $\begin{array}{l}\text { Geom. } \\
\text { mean }\end{array}$ & 18.68 & 11.08 & 23.96 & 16.80 & 12.84 & 19.97 & 29.64 & 14.52 & 39.21 & 16.72 & 11.32 & 20.80 \\
\hline $\begin{array}{l}\text { Coeff. } \\
\text { var }\end{array}$ & 328.86 & 288.59 & 579.32 & 241.99 & 200.96 & 407.84 & 233.92 & 179.95 & 366.76 & 195.01 & 151.20 & 297.93 \\
\hline \multicolumn{13}{|l|}{ Holiday } \\
\hline & Jutta & $\begin{array}{l}\text { Lower } \\
\text { conf. }\end{array}$ & $\begin{array}{l}\text { Upper } \\
\text { conf. }\end{array}$ & $\begin{array}{l}\text { Sebasti } \\
\text { an }\end{array}$ & $\begin{array}{l}\text { Lower } \\
\text { conf. }\end{array}$ & $\begin{array}{l}\text { Upper } \\
\text { conf. }\end{array}$ & Laura & $\begin{array}{l}\text { Lower } \\
\text { conf. }\end{array}$ & $\begin{array}{l}\text { Upper } \\
\text { conf. }\end{array}$ & My & $\begin{array}{l}\text { Lower } \\
\text { conf. }\end{array}$ & $\begin{array}{l}\text { Upper } \\
\text { conf. }\end{array}$ \\
\hline $\mathrm{N}$ & 64 & 64 & 64 & 71 & 71 & 71 & 58 & 58 & 58 & 108 & 108 & 108 \\
\hline Min & 1 & & & 1 & & & 1 & & & 1 & & \\
\hline Max & 1472 & & & 690 & & & 1788 & & & 1788 & & \\
\hline Sum & 5469 & 1763 & 8124 & 2923 & 1035 & 4322 & 5119 & 870 & 7850 & 6568 & 1846 & 9962 \\
\hline Mean & 85.45 & 27.55 & 126.94 & 41.17 & 14.58 & 60.87 & 88.26 & 15.00 & 135.34 & 60.81 & 17.09 & 92.24 \\
\hline $\begin{array}{l}\text { Std. } \\
\text { error }\end{array}$ & 25.94 & 0.00 & 35.54 & 12.08 & 0.00 & 16.68 & 32.42 & 0.00 & 45.18 & 19.32 & 0.00 & 26.43 \\
\hline $\begin{array}{l}\text { Varian } \\
\text { ce }\end{array}$ & $\begin{array}{l}43052 . \\
85\end{array}$ & $\begin{array}{l}- \\
19293 . \\
27\end{array}$ & $\begin{array}{l}80825 . \\
46\end{array}$ & $\begin{array}{l}10362 . \\
66\end{array}$ & $\begin{array}{l}- \\
3241.7 \\
9\end{array}$ & $\begin{array}{l}19763 . \\
72\end{array}$ & $\begin{array}{l}60976 . \\
27\end{array}$ & $\begin{array}{l}39240 . \\
86\end{array}$ & $\begin{array}{l}118373 \\
.90\end{array}$ & $\begin{array}{l}40331 \\
16\end{array}$ & $\begin{array}{l}- \\
19750 . \\
01\end{array}$ & $\begin{array}{l}75443 . \\
70\end{array}$ \\
\hline $\begin{array}{l}\text { Stand. } \\
\text { dev }\end{array}$ & 207.49 & 90.33 & 342.32 & 101.80 & 48.78 & 172.58 & 246.93 & 92.38 & 434.05 & 200.83 & 84.77 & 329.41 \\
\hline Median & 16.50 & 6.00 & 21.00 & 12.00 & 7.00 & 17.00 & 25.50 & 11.50 & 33.50 & 10.00 & 6.50 & 12.00 \\
\hline $\begin{array}{l}25 \\
\text { prentil }\end{array}$ & 7.25 & 3.25 & 9.50 & 5.00 & 3.00 & 7.00 & 9.75 & 3.00 & 13.50 & 4.00 & 2.00 & 5.00 \\
\hline $\begin{array}{l}75 \\
\text { prentil }\end{array}$ & 57.50 & -77.75 & 87.25 & 33.00 & 22.00 & 47.00 & 57.75 & 7.50 & 72.50 & 24.00 & 6.00 & 30.00 \\
\hline $\begin{array}{l}\text { Skewn } \\
\text { ess }\end{array}$ & 5.21 & 4.03 & 8.57 & 4.87 & 2.67 & 7.14 & 6.06 & 5.16 & 10.05 & 6.65 & 4.95 & 10.29 \\
\hline $\begin{array}{l}\text { Kurtosi } \\
\mathrm{s}\end{array}$ & 32.41 & 19.03 & 62.39 & 26.49 & -1.98 & 46.12 & 40.86 & 30.76 & 78.13 & 52.62 & 27.99 & 96.73 \\
\hline $\begin{array}{l}\text { Geom. } \\
\text { mean }\end{array}$ & 21.38 & 10.75 & 28.24 & 12.76 & 7.88 & 16.26 & 25.03 & 13.04 & 32.90 & 12.00 & 7.64 & 14.96 \\
\hline $\begin{array}{l}\text { Coeff. } \\
\text { var }\end{array}$ & 242.81 & 181.84 & 341.83 & 247.27 & 195.84 & 358.05 & 279.78 & 217.61 & 429.53 & 330.23 & 247.87 & 450.10 \\
\hline
\end{tabular}


Ottosen et al, 2020. Genet. Biodiv. J, Special issue (Behavioural Instability), 1-60

\section{Appendix J}

\section{Behavioural shifts}

\begin{tabular}{|c|c|c|c|c|c|c|c|}
\hline & & Sebas & & Laura & & My & \\
\hline & & Chi2 & $\mathrm{P}$ & Chi2 & $\mathrm{P}$ & Chi2 & $\mathrm{P}$ \\
\hline Control & Jutta & 9.83 & 0.080 & 10.18 & 0.071 & 3.62 & 0.61 \\
\hline & Sebastian & & & 23.55 & $\begin{array}{l}0.00027 \\
* *\end{array}$ & 5.44 & 0.37 \\
\hline & Laura & & & & & 16.69 & $\begin{array}{l}0.0051 * \\
*\end{array}$ \\
\hline & My & & & & & & \\
\hline Holiday & Jutta & 11.15 & $0.049 *$ & 14.83 & $0.011^{*}$ & 12.17 & $0.033^{*}$ \\
\hline & Sebastian & & & 17.15 & $\begin{array}{l}0.0042 * \\
*\end{array}$ & 11.32 & $0.045^{*}$ \\
\hline & Laura & & & & & 41.67 & $\begin{array}{l}6.88 * 10 \\
\wedge-8 * *\end{array}$ \\
\hline & My & & & & & & \\
\hline
\end{tabular}

$* P<0.05$

$* * P<0.0$ 
